\title{
Fundus characterization for automatic disease screening through retinal image processing
}

SANDRA MORALES MARTÍNEZ 


\title{
Fundus characterization for automatic disease screening through retinal image processing
}

\author{
Sandra Morales Martínez
}

PhD Thesis

July, 2015

Departamento de Ingeniería Electrónica 


\section{Fundus characterization for automatic disease screening through retinal image processing}

Author: Sandra Morales Martínez

Supervisor: Dr. Valery Naranjo Ornedo

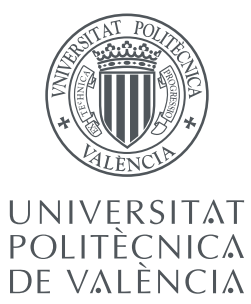

Departamento de Ingeniería Electrónica

July, 2015 
Collection doctoral thesis

(c) Sandra Morales Martínez

(c) 2015, of the present edition: Editorial Universitat Politècnica de València Telf.: 963877012 / www.lalibreria.upv.es

ISBN: 978-84-9048-430-2 (printed version)

Any unauthorized copying, distribution, marketing, editing, and in general any other exploitation, for whatever reason, of this piece of work or any part thereof, is strictly prohibited without the authors' expressed and written permission. 
The research derived from this doctoral thesis was partially funded by the Instituto de la Mediana y Pequeña Industria Valenciana (IMPIVA), Project IMIDTA/2010/47, by the Ministerio de Economía y Competitividad of Spain, Project ACRIMA (TIN2013-46751-R), and by NILS Science and Sustainability Programme (010-ABEL-IM-2013). 



\section{Acknowledgments}

La presentación de esta tesis es el resultado de un largo camino en el que ha intervenido un gran número de personas. Unos han estado de principio a fin, otros aparecieron durante su recorrido y algunos no han podido verlo acabar. Sin embargo, todos ellos han contribuido, de una u otra forma, a que la tesis haya cogido forma y a que yo sea como soy, como investigadora y como persona. Por tanto, creo que es buen momento para darles las gracias.

Me gustaría dedicar mis primeras palabras a Valery, mi directora de tesis, mi maestra, mi amiga y compañera en muchos ámbitos, no sólo académicos. Gracias por haber visto en mí tus mismas ganas por el trabajo y por el modo de hacerlo. Gracias por escucharme, por ayudarme en todo momento, por tus consejos y por aportarme algo nuevo en cada conversación. Puedo decir, aunque esta tesis haya llegado a su fin, que estoy orgullosa de nuestro trabajo y que aún nos queda mucho por hacer.

Muchas gracias también a todos los LabHumanos: Pablo, Rober, Fran M., Miguel, Eliseo, Félix, Fran P., Jorge, Adri B.; y, en especial, a Fer, Adri y Juanjo, con los que he tenido la suerte de trabajar de un modo más cercano. Gracias a todos por haber hecho más fácil el día a día siendo unos compañeros extraordinarios pero, también, buenos amigos. Siempre que nos reunamos seguiremos recordando, una y otra vez, las anécdotas ocurridas durante estos años.

I would like to express my deep gratitude to Kjersti Engan for her supervision during my stay in the University of Stavanger (UiS), for introducing me in the Norwegian culture and for treating me like a friend. Tusen takk. See you in Valencia, Stavanger or wherever.

Gracias a la Dra. Amparo Navea y a su equipo de la FISABIOOftalmología Médica (FOM) por creer en el proyecto en el que se enmarca esta tesis y por su colaboración durante este tiempo. Gracias 
por facilitarnos imágenes de fondo de ojo, las cuales han sido clave para la validación de algunos puntos de la tesis, y por resolver las dudas que han ido surgido desde el punto de vista clínico.

Finalmente, gracias a mi madre y al resto de mi familia y amigos que han estado, están y estarán incondicionalmente durante éste y todos los proyectos que emprenda en mi vida. No puedo dejar de agradecer a Edu su apoyo constante y comprensión en la fase final de esta andadura, la cual será el principio de otras. 
"Nothing in life is to be feared, it is only to be understood. Now is the time to understand more, so that we may fear less."

Marie Curie 



\section{Abstract}

The World Health Organization estimates that in 2010 there were 285 million people visually impaired in the world. It is calculated that $80 \%$ of these cases are preventable or treatable. In addition, an aging population and an increase in chronic diseases are two factors based on which a higher number of blindness cases is predicted in the future. Hypertension, diabetic retinopathy (DR), age-related macular degeneration (AMD) and glaucoma are the most common pathologies in the current society that provoke retinal damage and can be directly related to blindness and vision loss. The early diagnosis of these diseases allows, through appropriate treatment, to reduce costs generated when they are in advanced states and may become chronic. This fact justifies screening campaigns. However, a screening campaign requires a heavy workload for trained experts in the analysis of anomalous patterns of each disease, which in addition to the increase in population at risk, makes these campaigns economically unfeasible. Therefore, the need for automatic screening system developments is highlighted.

The final goal of this thesis is to develop novel methods that allow the analysis and processing of fundus images to implement an automatic screening of four of the most important diseases affecting world population. In particular, the main objective of the thesis is to build up algorithms for the characterization of the retinal structures and the retina background in order to assist in the discrimination between a "normal" and pathological retina.

Mathematical morphology along with other operators are used for the detection of the retinal vessels and the optic disk. The proposed methods work properly on databases with a large degree of variability. Not only have the main structures been segmented, but significant features have also been extracted to be used in a computer aided diagnosis software for hypertensive risk determination. The texture of the retina background is also analyzed in this work by means of local binary patterns with the aim of identifying DR and AMD and avoiding 
the need for segmentation of the characteristic retinal lesions of each disease. The results are promising, above all for AMD diagnosis. 


\section{Resumen}

La Organización Mundial de la Salud estima que en 2010 había 285 millones de personas con alguna discapacidad visual en el mundo. Se calcula que el $80 \%$ de estos casos son evitables o tratables. Además, el envejecimiento de la población y el aumento de las enfermedades crónicas son dos factores que hacen prever un número todavía mayor de casos de ceguera en el futuro. La hipertensión, la retinopatía diabética (RD), la degeneración macular asociada a la edad (DMAE) y el glaucoma son las enfermedades más comunes que provocan daños en la retina y, por tanto, están directamente relacionadas con la ceguera y con la pérdida de visión. El diagnóstico de estas enfermedades en estadios tempranos permite, mediante el tratamiento adecuado, reducir los costes que generan en estados ya avanzados y que en la mayoría de los casos acaban convirtiéndose en crónicas, lo que justifica la realización de campañas de cribado. Sin embargo, una campaña de cribado exige una gran carga de trabajo de personal experto entrenado en el análisis de los patrones anómalos propios de cada enfermedad, lo que sumado al aumento de la población de riesgo, hace que estas campañas sean inviables económicamente. Por lo tanto, se evidencia la necesidad del desarrollo de sistemas de cribado automáticos.

El objetivo final del presente trabajo es la implementación de métodos novedosos de análisis de imágenes de fondo de ojo para usarlos en un sistema de cribado de cuatro de las enfermedades más importantes que afectan a la población actual. En concreto, el objetivo principal de la tesis es el desarrollo de algoritmos para la caracterización de las estructuras y del fondo retiniano, los cuales servirán de ayuda para discriminar una retina "normal" de otra patológica.

Para la detección de los vasos retinianos y del disco óptico, se ha usado morfología matemática además de otros operadores. Se ha demostrado que los métodos propuestos para este fin funcionan adecuadamente en bases de datos con un alto grado de variabilidad. No sólo se han segmentado las principales estructuras retinianas, sino 
que, además, se han extraído sus características más significativas para determinar el riesgo hipertensivo. En este trabajo, también se han analizado las texturas presentes en el fondo de la retina por medio de la teoría de los patrones binarios locales con el objetivo de identificar la RD y la DMAE a la vez que se evita la necesidad de la segmentación de las lesiones específicas de cada enfermedad. Los resultados son prometedores, sobre todo, para la detección de la DMAE. 


\section{Resum}

L'Organització Mundial de la Salut estima que en 2010 havia 285 milions de persones amb alguna discapacitat visual en el món. Es calcula que el $80 \%$ d'aquests casos són evitables o tractables. A més, l'envelliment de la població i l'augment de les malalties cròniques són dos factors que fan preveure un número encara major de casos de ceguera en el futur. La hipertensió, la retinopatia diabètica (RD), la degeneració macular associada a l'edat (DMAE) i el glaucoma són les malalties més comuns que provoquen danys en la retina i, per tant, estan directament relacionades amb la ceguera $\mathrm{i}$ amb la pèrdua de visió. El diagnòstic d'aquestes malalties en estadis primerencs permet, per mitjà del tractament adequat, reduir els costos que generen en estats ja avançats i que en la majoria dels casos acaben convertintse en cròniques, la qual cosa justifica la realització de campanyes de garbellament. No obstant això, una campanya de garbellament exigix una gran càrrega de treball de personal expert entrenat en l'anàlisi dels patrons anòmals propis de cada malaltia, que si es suma a l'augment de la població de risc, fa que aquestes campanyes siguen inviables econòmicament. Per tant, s'evidencia la necessitat del desenrotllament de sistemes de garbellament automàtics.

L'objectiu final del present treball és la implementació de mètodes nous d'anàlisi d'imatges de fons d'ull per a usar-los en un sistema de garbellament de quatre de les malalties més importants que afecten la població actual. En concret, l'objectiu principal de la tesi és el desenvolupament d'algoritmes per a la caracterització de les estructures i del fons retinià, els quals serviran d'ajuda per a discriminar una retina "normal" d'una altra patològica.

Per a la detecció dels vasos retinians i del disc òptic, s'ha usat morfologia matemàtica a més d'altres operadors. S'ha demostrat que els mètodes proposats per a aquest fi funcionen adequadament en bases de dades amb un alt grau de variabilitat. No sols s'han segmentat les principals estructures retinianes, sinó que, a més, s'han extret les seues 
característiques més significatives per a determinar el risc hipertensiu. En aquest treball, també s'han analitzat les textures presents en el fons de la retina per mitjà de la teoria dels patrons binaris locals amb l'objectiu d'identificar la RD i la DMAE al mateix temps que s'evita la necessitat de la segmentació de les lesions específiques de cada malaltia. Els resultats són prometedors, sobretot, per a la detecció de la DMAE. 


\section{Contents}

1 Introduction 1

1.1 Motivation .................. . . 3

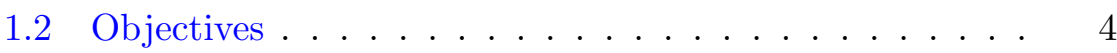

1.3 Main contributions . . . . . . . . . . . . 5

1.4 Thesis framework . . . . . . . . . . . . . 6

1.5 Outline ................... 7

2 The retina 11

2.1 Retina's anatomy . . . . . . . . . . . . . . 13

2.2 Pathologies related to the retina . . . . . . . . 15

2.2.1 Hypertensive retinopathy . . . . . . . . . . . . 15

2.2 .2 Diabetic retinopathy . . . . . . . . . . . . . 18

2.2.3 Age-related macular degeneration . . . . . . . . . 20

2.2 .4 Glaucoma . . . . . . . . . . . . . . . 21

2.3 Relevant groups . . . . . . . . . . . . . . . 23

3 Retinal structure characterization $\quad \mathbf{2 7}$

3.1 Introduction . . . . . . . . . . . . . . . 31

3.2 Theoretical background . . . . . . . . . . . . . 34

3.2.1 Morphological operators . . . . . . . . . . . 34

3.2.2 Stochastic watershed transformation . . . . . . 39

3.3 Vessel segmentation . . . . . . . . . . . . . . 43

3.3.1 Vessel extraction . . . . . . . . . . . . . . 45

3.3 .2 Centerline extraction . . . . . . . . . . . 50

3.4 Significant points . . . . . . . . . . . . . 56 
3.4.1 Terminal and bifurcation points . . . . . . . 58

3.4 .2 Crossing points . . . . . . . . . . . . . 60

3.5 Optic disk segmentation . . . . . . . . . . . . 63

3.5.1 Pre-processing . . . . . . . . . . . . 66

3.5.2 Processing . . . . . . . . . . . . . . 71

3.5 .3 Post-processing . . . . . . . . . . . . . 78

3.6 Application to pathology detection: Hypertensive risk . 79

3.6.1 Pre-processing . . . . . . . . . . . . . 81

3.6 .2 Processing . . . . . . . . . . . . . . . 82

3.6.3 Post-processing . . . . . . . . . . . . . . . 83

3.7 Results . . . . . . . . . . . . . . . 86

3.7 .1 Material . . . . . . . . . . . . 86

3.7.2 Retinal vessel segmentation . . . . . . . . . . 89

3.7.3 Retinal vessel centerline . . . . . . . . . . . . 94

3.7 .4 Optic disk . . . . . . . . . . . . . . 95

3.7.5 Computer-aided diagnosis software for hypertensive risk determination . . . . . . . . . . . . 112

3.8 Conclusions . . . . . . . . . . . . . . . . . . . 115

4 Retina background characterization $\quad 117$

4.1 Introduction . . . . . . . . . . . . . . . . . 119

4.2 Theoretical background . . . . . . . . . . . . . . 121

4.3 Application to pathology detection: Diabetic retinopathy and age-related macular degeneration . . . . . . . . 124

4.3.1 General description . . . . . . . . . . . . . . . 124

4.3 .2 Feature extraction . . . . . . . . . . . . . 124

4.3 .3 Classification . . . . . . . . . . . . . 125

4.4 Results. . . . . . . . . . . . . . . . . . 130

4.4 Material . . . . . . . . . . . . . 130

4.4 .2 Experiments . . . . . . . . . . . . . 134

4.5 Conclusions . . . . . . . . . . . . . . . . . 139

5 Conclusions $\quad 141$

$\begin{array}{ll}\text { Merits } & 145\end{array}$ 



\section{List of Figures}

1.1 Thesis framework flowchart. . . . . . . . . . . 9 9

2.1 Eyeball anatomy . . . . . . . . . . . . . . . . 13

2.2 Main parts of the retina . . . . . . . . . . 15

2.3 Effects of different pathologies on the vision . . . . . . 16

2.4 Some retinal vascular signs . . . . . . . . . . . 17

2.5 Features of diabetic retinopathy . . . . . . . . . . . 19

2.6 Grades of diabetic retinopathy . . . . . . . . . . . 19

2.7 Signs of age-related macular degeneration . . . . . . . 21

2.8 Glaucoma effects on the cup-to-disk ratio . . . . . . . 22

3.1 Basic morphological operators . . . . . . . . . . . 35

3.2 Basic morphological filters . . . . . . . . . . . . . . . . 36

3.3 Top-hat transformation . . . . . . . . . . . . . 37

3.4 Supremum of openings . . . . . . . . . . . . . . . 38

3.5 Geodesic reconstruction . . . . . . . . . . . . . . 39

3.6 Close-hole operator . . . . . . . . . . . . . . . . . . . 40

3.7 Over and under-segmentation problems of the watershed transformation ..................... 41

3.8 Probability density function . . . . . . . . . . . . . . 44

3.9 RGB to gray conversion . . . . . . . . . . . . . . 45

3.10 Image enhancement . . . . . . . . . . . . . . 46

3.11 Small opening . . . . . . . . . . . . . . . . 47

3.12 Principal curvature computation . . . . . . . . . . . 48

3.13 Final vessel segmentation steps . . . . . . . . . . . . 49

3.14 Flowchart for skeleton extraction. . . . . . . . . . . 51 
3.15 First steps of the skeleton extraction process . . . . . 52

3.16 Use of the stochastic watershed for vascular skeleton extraction . . . . . . . . . . . . . 5 53

3.17 Stochastic watershed on the crossing of two vessels . . . 54

3.18 Final steps of the skeleton extraction process . . . . . 54

3.19 Pruning process . . . . . . . . . . . . . 55

3.20 Example of a composite structuring element. . . . . . . 57

3.21 Structuring elements used for detecting terminal points. 58

3.22 Structuring elements used for detecting bifurcation points. 58

3.238 -connectivity to $m$-connectivity conversion . . . . . 59

3.24 Structuring elements used to convert an 8-connected skeleton to $m$-connectivity. . . . . . . . . . . 60

3.25 Structuring elements used for detecting simple crossing points. . . . . . . . . . . . . 6 61

3.26 Different types of crossing points . . . . . . . . . 61

3.27 Vessel intersection . . . . . . . . . . . . . . 62

3.28 Automatic detection of common intersections . . . . . 64

3.29 Optic disk segmentation diagram. . . . . . . . . . . . 66

3.30 Principal components . . . . . . . . . . . . . . 69

3.31 OD pre-processing . . . . . . . . . . . . . 72

3.32 Gray-image centroid on $z_{1} \ldots \ldots \ldots \ldots 74$

3.33 Gray-image centroid on the residue of close operator . . 74

3.34 Final markers . . . . . . . . . . . . . . 75

3.35 Probability density function . . . . . . . . . 76

3.36 Last marker-controlled watershed . . . . . . . . . . 77

3.37 Region discrimination . . . . . . . . . . . . . . 78

3.38 OD post-processing . . . . . . . . . . . . . . . 79

3.39 Block diagram of the presented tool. . . . . . . . . . 81

3.40 Bifurcation angle measurement . . . . . . . . . . 84

3.41 Bifurcation angles measured on two different image areas 84

3.42 Vessel caliber measurement . . . . . . . . . . . . 85

3.43 Image processing performed by the developed software . 85

3.44 Some vessel segmentation results on DRIVE database . 91

3.45 Vessel segmentation comparison . . . . . . . . . . . 92

3.46 Effect of the input image in the segmentation method . 93 
3.47 Skeleton results of the method proposed in Subsection 3.3.294

3.48 Comparison between different methods on a DRIVE image 96

3.49 Comparison between different methods on a STARE image 97

3.50 Sub and over-segmentation case . . . . . . . . . . 99

3.51 Some OD segmentation results on DRIONS database . . 101

3.52 Some OD segmentation results on DIARETDB1 database 102

3.53 Some OD segmentation results on DRIVE database . . 103

3.54 Some OD segmentation results on MESSIDOR database 104

3.55 Some OD segmentation results on ONHSD database . . 105

3.56 Advantages of PCA . . . . . . . . . . . . 110

3.57 Under-segmentation problem produced in other markercontrolled-watershed method . . . . . . . . . . . . 111

3.58 Robustness of the gray-image centroid in presence of exudates . . . . . . . . . . . . . 112

4.1 Fundus images . . . . . . . . . . . . . . . . . . . . 119

4.2 LBP neighborhoods . . . . . . . . . . . . . . 122

4.3 LBP computation . . . . . . . . . . . . . . . 123

4.4 Feature extraction using $P=8$ and $R=5 \ldots \ldots 126$

4.5 Feature extraction flowchart . . . . . . . . . . 127

4.6 External cross validation flowchart . . . . . . . . . . 129

4.7 Final validation flowchart. . . . . . . . . . . . . 130

4.8 Fundus images belonging to the different used databases 132

4.9 Excluded images . . . . . . . . . . . . . . . 133 



\section{List of Tables}

3.1 Comparison between the results of the method proposed in Subsection 3.3.1 and the $2^{\text {nd }}$ observer of DRIVE database 90

3.2 True positives/negatives and false positives/negatives . 90

3.3 Accuracy of several methods on DRIVE database . . . . 92

3.4 Results obtained by the method proposed in Subsection $3.5 \ldots \ldots \ldots \ldots \ldots \ldots$

3.5 True positives/negatives and false positives/negatives . 99

3.6 Success rate in the optic disk location . . . . . . . . 100

3.7 Comparison of the parameters of Table 3.4 achieved by the method proposed in Section 3.5, by the $2^{\text {nd }}$ observer and by other marker-controlled-watershed algorithm . . 106

3.8 Comparison of the method proposed in Section 3.5 with the work presented in (Welfer et al., 2010) . . . . . . . 107

3.9 Subjective classification of performance on ONHSD dataset. . . . . . . . . . . . . . . 109

3.10 Comparison between the use of PCA versus the use of the red component . . . . . . . . . . . . 110

4.1 Content of each database. . . . . . . . . . . . . . . 134

4.2 Content of the model and validation sets. . . . . . . . . 134

4.3 Results of the different experiments on the model set (external CV). . . . . . . . . . . . 136

4.4 Results of the different experiments on the validation set. 137

4.5 AMD-Normal comparison on different databases. . . . . 138

4.6 DR-Normal comparison on different databases. . . . . . 138 



\section{List of Algorithms}

3.1 Vessel segmentation . . . . . . . . . . . . . . 50

3.2 Vessel centerline extraction . . . . . . . . . . . . . 56

3.3 Significant point detection . . . . . . . . . . . . . 65

4.1 Feature extraction . . . . . . . . . . . . . 128 



\section{Chapter 1}

\section{Introduction}

This chapter presents the motivations behind this thesis, its objectives and its main contributions. In addition, it also introduces the thesis framework and the thesis outline.

\section{Contents}

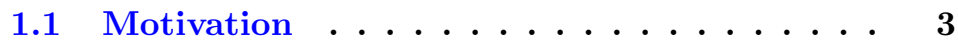

1.2 Objectives .............. 4

1.3 Main contributions . . . . . . . . 5

1.4 Thesis framework . . . . . . . . 6

1.5 Outline .............. 7 



\subsection{Motivation}

Retinal imaging is nowadays a mainstay of the clinical care and management of patients with retinal as well as systemic diseases. Many important diseases manifest themselves in the retina and originate either in the eye, the brain or the cardiovascular system. In particular, the most prevalent diseases that can be studied via eye imaging and image analysis are: diabetes, diabetic retinopathy, age-related macular degeneration, glaucoma and cardiovascular disease (Abràmoff et al., 2010).

The World Health Organization (WHO) estimates that in 2010 there were 285 million people visually impaired around the world (World Health Organization, 2013). In spite of the fact that the number of blindness cases has been significantly reduced in recent years, it is estimated that $80 \%$ of the cases of visual impairment are preventable or treatable (World Health Organization, 2013).

Screening campaigns are usually considered effective if the disease is identified at an early, preferably preclinical, stage and if it is amenable to treatment (Cree and Jelinek, 2011). The early diagnosis allows, through appropriate treatment, to reduce costs generated when the diseases are in advanced states and may become chronic. In the main, the best approach for the assessment of the population at-risk seems to be a direct, regular and complete ophthalmologic examination (Herbert et al., 2003). However, a screening campaign requires a large workload of trained experts in the analysis of anomalous patterns of each disease, which in addition to population growth, ageing, physical inactivity and rising levels of obesity make these campaigns economically infeasible because the number of ophthalmologists for the assessment by direct examination of the population at-risk is high (Verma et al., 2002). Moreover, it must be stressed that this type of retinal disease identification that is based on manual observation, is highly subjective (Anitha et al., 2011). So, for those reasons, the need for automatic screening systems is highlighted and they have 
become increasingly important. Thanks to high resolution of the current retinal cameras, the fundus images can be automatically processed by a screening software providing invaluable help to clinicians in diagnosis and disease prevention. Automatic screening systems are able to relieve physicians of repetitive work, increase efficiency and provide remarkable cost savings in addition to be accessible in rural and remote areas where there is a lack of eye specialists (American Diabetes Association, 2008).

\subsection{Objectives}

The final goal of this thesis is the development of new methods that allow the analysis and processing of fundus images for using this information in the implementation of an automatic screening of four of the most important diseases that affect world population: hypertension, diabetic retinopathy, age-related macular degeneration and glaucoma. In particular, the main objective of the thesis is to build up algorithms for the characterization of the retinal structures and the retina background in order to assist in the discrimination between a "normal" and pathological retina. For that, several methods and methodologies have been carried out, which will be further explained. From the main objective, several secondary objectives arise:

- Determination of the characteristic patterns of each disease under study.

- Development and implementation of methods for the location of the main structures of the retina on fundus images which can be related to several pathologies.

- Development and implementation of methods for the extraction of determinant features of the fundus images for diagnosis.

- Selection of a model for the classification of the fundus images as belonging to healthy subject or suspicious of some of the pathologies under study. 


\subsection{Main contributions}

This thesis provides novel methods to characterize fundus images by means of advanced image processing techniques. One of its main contributions is to perform an in-depth study about the use of mathematical morphology in order to detect the most significant retinal structures: vascular network and optic disk. The methods presented in the Chapter 3 are defined by combining different morphological operators, basic ones and other more complex, and by making use of a variant of the watershed transformation, the stochastic watershed, which avoids over and under-segmentation problems related to the classical watersheds.

The vascular network is characterized in two different ways, by means of the segmentation of the complete vasculature and through the vessel centerline extraction. Both approaches are based on mathematical morphology but also use principal curvature information. On the one hand, the main advantage of the method proposed for complete vasculature segmentation is that the optic disk border is not misclassified as vessel which would provoke vessel tracking problems (Morales et al., 2012). On the other hand, the benefit of directly extracting the vessel centerline through the presented algorithm, instead of after a skeletonization process, is that the dependence of previous stages is avoided at the same time that its robustness is kept (Morales et al., 2014a).

The method developed for optic disk segmentation is also based on mathematical morphology although includes a principal component analysis (PCA) in the pre-processing stage to deal with the problem of RGB to gray conversion. It is demonstrated that the use of PCA improves the results of the red component, which is the component most used in the state of the art for the optic disk detection. The extensive validation of this method must also be highlighted. Its results are compared with 5 public databases which shows the high reliability of the algorithm (Morales et al., 2013). 
Other contribution of the thesis is that not only are the main retinal structures (blood vessels and optic disk) segmented, but the vascular network is also characterized through the detection of its significant points and the computation of the vessel caliber and the bifurcation angles. These features are used in a computer-aided diagnosis software for hypertensive risk determination which increases the percentage of cases detected if it is compared with the diagnosis obtained from the visual inspection of the fundus (Morales et al., 2014b).

In addition to the hypertension risk determination, the thesis focuses also on the diagnosis of diabetic retinopathy and age-related macular degeneration. The last contribution of the thesis is related to the detection of these diseases. Instead of relying on the segmentation of the characteristic retinal lesions of each disease as most of state-of-theart works, the diagnosis is based on a texture analysis of the retina background followed by machine learning techniques which achieves promising results.

\subsection{Thesis framework}

This thesis stands within the framework of two research projects: "Study of retinal microvascular architecture through fundus image processing acquired by non-mydriatic retinal cameras" (IMIDTA/2010/47) and "Fundus image processing for automatic screening of ophthalmological diseases - ACRIMA" (TIN2013-46751-R). These projects were or are being funded by the Instituto de la Mediana y Pequeña Industria Valenciana (IMPIVA) and by the Ministerio de Economía y Competitividad of Spain, respectively.

The first one was carried out thanks to the collaboration among LabHuman (Univesitat Politècnica de València), the Department of Pediatrics of the Hospital General de Valencia and the Fundación Oftalmológica del Mediterráneo. The aim of this project was the study of retinal microvascular architecture by measuring the bifurcation angles and the caliber of the retinal arterioles in children with low 
birth weight, as a prognostic marker of hypertension and cardiovascular pathology. For that purpose, a computer-aided diagnosis software, able to segment blood vessels and perform angle and caliber measurements, was developed. This project supposed the beginning of a research line in LabHuman about retinal image processing.

The scope of ACRIMA is wider than the previous project. Its objective is to develop an automatic screening system for the three most significant diseases related to visual impairment: glaucoma, diabetic retinopathy and age-related macular degeneration. At present, there is no screening system as the proposed in the project, which combines the screening of these three diseases. The members of the Fundación Oftalmológica del Mediterráneo, renamed nowadays as FISABIO-Oftalmología Médica, continue collaborating with LabHuman in this project.

Both projects have a common denominator. The resulting systems are designed to be used by general practitioners (GP) as computeraided diagnosis tools. When a patient goes to a medical consultation, the GP will determine the need for medical tests. If they are needed, a fundus image of the patient will be captured and processed by the developed tools. If the tool detects that there is pathology risk, the patient will be sent to the ophthalmologist who will decide if some specific tests are needed and will determine the final diagnosis. This process is summarized in Figure 1.1.

\subsection{Outline}

This thesis is divided in 5 chapters. This chapter has presented the motivations behind the research involved in the thesis, the main and secondary objectives and its main contributions. Finally, the thesis framework is also explained.

Chapter 2 introduces the anatomical aspects of the retina, the most important retinal diseases and the most relevant groups working on the 
development of screening systems for the detection of some of these diseases.

Chapter 3 presents new methods to detect the retinal vessels and the optic disk, two of the most important anatomical structures of the fundus, and to determine some of their main characteristics. These elements will be key for pathology detection.

In Chapter 4, the texture of the retina background is analyzed by means of local binary patterns to differentiate between pathological and healthy images. With this analysis, it is intended to determine if a patient is suspicious of pathology but avoiding the need for segmentation of different types of lesions.

General and final conclusions in addition to future prospects are presented in Chapter 5. Next, the scientific publications derived from this thesis are cited. 


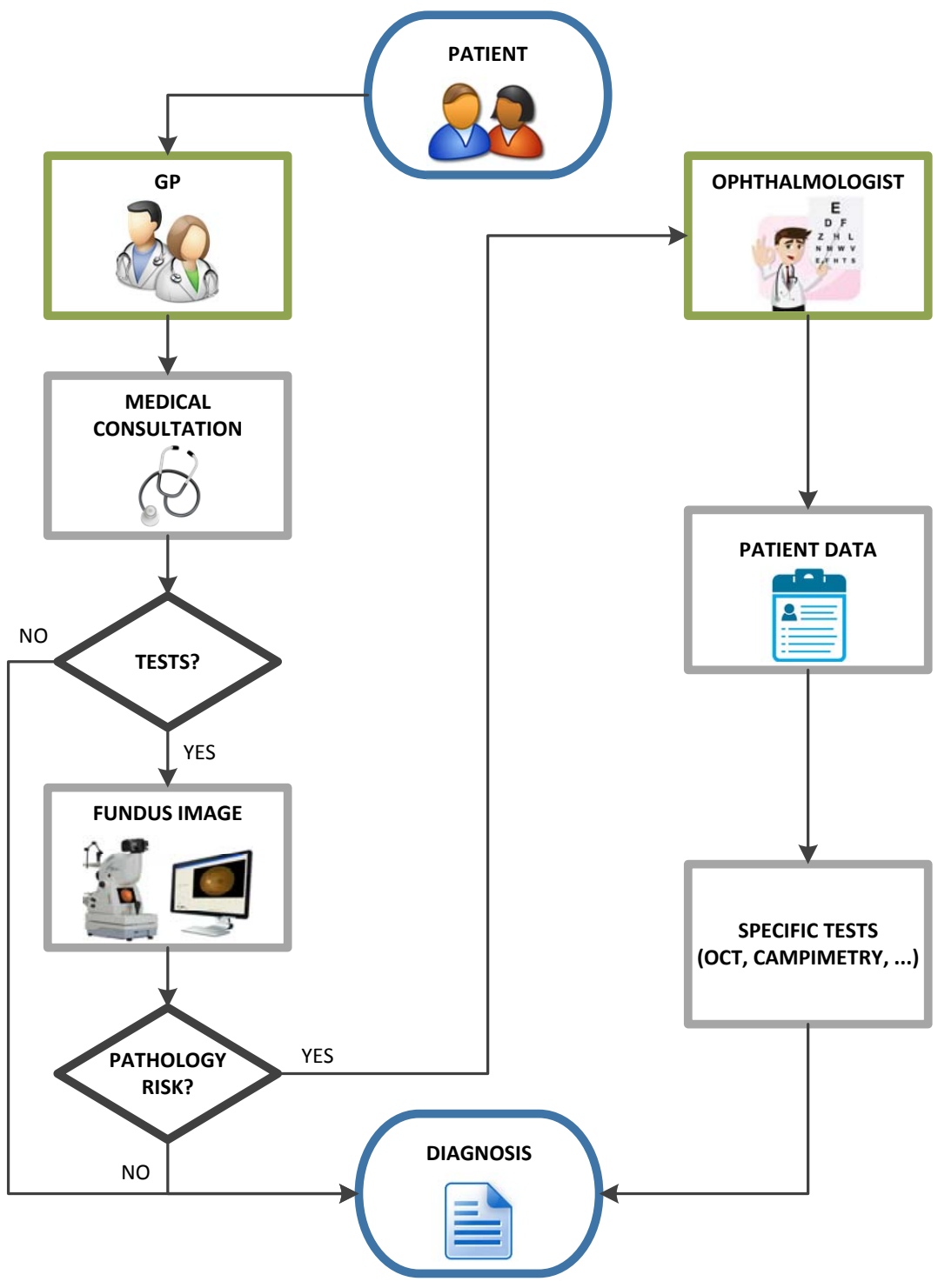

Figure 1.1: Thesis framework flowchart. 



\section{Chapter 2}

\section{The retina}

This chapter presents a brief explanation about anatomical aspects of the retina, the most important retinal diseases and the most relevant groups working on the development of screening systems for the detection of some of these diseases.

\section{Contents}

2.1 Retina's anatomy ........... 13

2.2 Pathologies related to the retina . . . . 15

2.2.1 Hypertensive retinopathy . . . . . . . . . 15

2.2.2 Diabetic retinopathy . . . . . . . . . . 18

2.2.3 Age-related macular degeneration . . . . . . 20

2.2.4 Glaucoma . . . . . . . . . . . . . 21

2.3 Relevant groups ............ 23 



\subsection{Retina's anatomy}

The eye is the sense organ of sight. It is often compared to a camera which captures and transmits light through a lens to create an image.

The eyeball consists of three coats: the sclera, with its anterior part, the cornea; the uvea that includes the choroid, ciliary body and iris; and the retina (Figure 2.1). The retina is the innermost layer and the focus of this thesis. The purpose of the retina is to transform light into a nerve impulse.

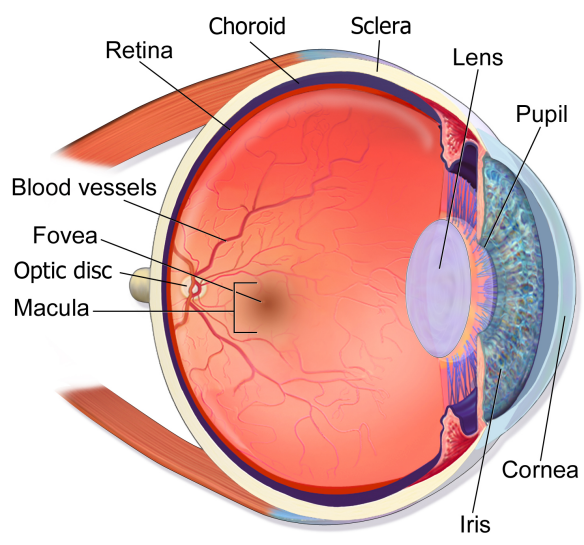

Figure 2.1: Eyeball anatomy. ${ }^{1}$

Approximately 1.2 million axons of ganglion cells converge to form the optic nerve. The optic nerve is directly connected to the retina. Its function is to send the nerve impulses produced by the retina to the brain to be interpreted and transformed into a visual perception.

The retina is made up two kinds of photoreceptors (cones and rods). The cones are responsible for bright light and colors and the rods for peripheral and night vision. There are 5 million cones and 125 million rods in an average retina.

\footnotetext{
${ }^{1}$ Image adapted from Blausen gallery 2014 - Wikiversity Journal of Medicine (http://en.wikiversity.org/wiki/Blausen_gallery_2014).
} 
Next, the most important structures of the retina will be detailed. They can be observed in Figure 2.2.

- Blood vessels.

Blood vessels supply the retina. They enter and exit to and from the retina via the optic disk. The retinal vascular network is composed by arteries and veins. The main branches of the central retinal artery and vein form four vascular arcades.

- Optic disk.

The optic disk is a circular to oval area where the optic nerve is connected to the retina. It measures about $2 \times 1.5 \mathrm{~mm}$ across and can be seen as a bright yellow disk. It is also called blind spot because there are not receptors in this part of the retina.

In the center of the optic disk there is a white depression with cup-like shape called optic cup. The optic cup is not occupied by nerve fibers, i.e., devoid of neuroretinal tissue. The neuroretinal rim is the area the optic disk which contains the neural elements and is located between the edge of the disk and the optic cup. The cup-to-disk ratio is important clinically because its increase indicates the death of ganglion cells.

- Macula.

The macula is a pigmented area near the center of the retina that is responsible for finest central vision. It is about $1.5 \mathrm{~mm}$ in diameter and it is located two disk diameter temporal to optic disk. The normal macula appears darker than the rest of the retina.

- Fovea.

The fovea is a small pit (approximately $500 \mu \mathrm{m}$ in diameter) involved with high acuity vision. It is located in the center of the macula and contains a high density of cones. Rods are present everywhere in the retina except at the fovea. 


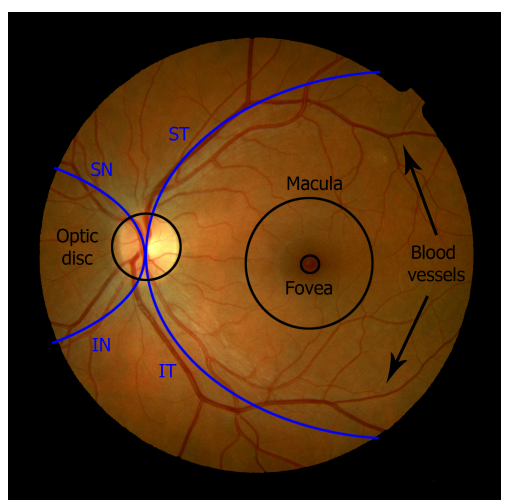

Figure 2.2: Main parts of the retina: blood vessels, optic disk, macula and fovea. The vascular arcades formed by the blood vessels are marked in blue: superotemporal (ST), inferotemporal (IT), superonasal (SN) and inferonasal (IN).

\subsection{Pathologies related to the retina}

Due to the retina's vital role in vision, the damage of the retina can cause visual impairment and, even, permanent blindness. Hypertension, diabetic retinopathy, age-related macular degeneration and glaucoma are some of the most common diseases in the current society that provoke retinal damage and are detectable through fundus inspection (World Health Organization, 2010a; Abràmoff et al., 2010). Next, these pathologies will be described, emphasizing the characteristic patterns of the disease and summarizing the works found in the literature for their detection. By way of introduction, in Figure 2.3 the effects of some diseases on the vision are shown.

\subsubsection{Hypertensive retinopathy}

Hypertension is a chronic disease characterized by a continuous increase in the blood pressure in the arteries. It is one of the major cardiovascular risk factors. Globally, the overall prevalence of high blood pressure in adults aged 25 and above was around $40 \%$ in 2008 


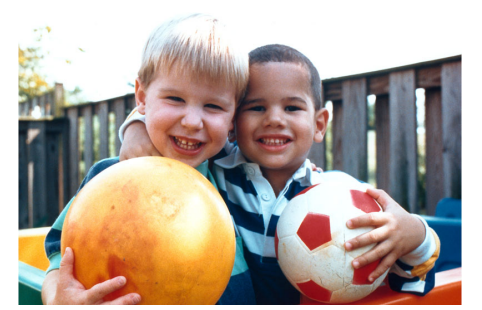

(a)

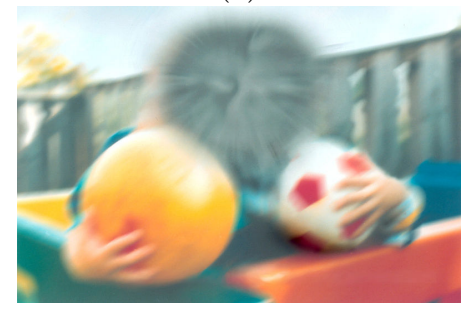

(c)

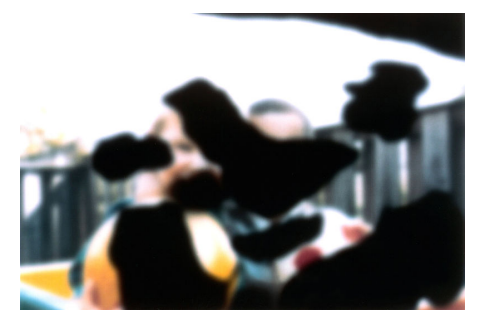

(b)

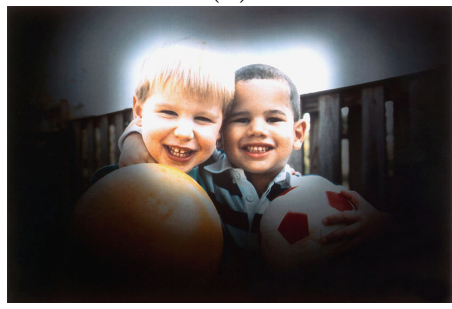

(d)

Figure 2.3: Effects of different pathologies on the vision: (a) Normal vision, (b) With diabetic retinopathy, (c) With age-related macular degeneration and (d) With glaucoma. ${ }^{2}$

(World Health Organization, 2010b). This data is directly related to hypertension prevalence.

Hypertension results from a complex interaction of genes and environmental factors as overweight and salt and alcohol intake. Moreover, as blood pressure rises with aging, the risk of becoming hypertensive increases in later life (Vasan et al., 2002).

Although hypertension is rarely accompanied by visual symptoms, it can be suspected on the basis of the presence of hypertensive retinopathy since changes in the arterial hypertension of the retina are indicator of the state of the blood vessels in other organs (Wong and Mitchell, 2007). In particular, vascular changes produced in

\footnotetext{
${ }^{2}$ Images provided by the National Eye Institute (www.nei.nih.gov).
} 
systemic diseases usually induce specific modifications in the retinal vessels, such as: generalized retinal-arteriolar narrowing which implies a decrease in the ratio between the diameter of retinal arteries and veins, arteriovenous nicking, focal arteriolar narrowing, alteration in the arteriolar light reflex, arteriolar tortuosity, an increase in the angle of arteriolar branching, central or branch retinal artery and vein occlusions, etc. (Wong et al., 2001; Liew and Wang, 2011). Some of these signs can be observed in Figure 2.4.

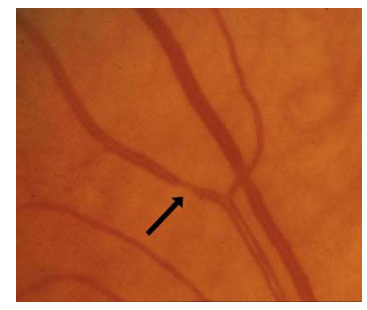

(a)

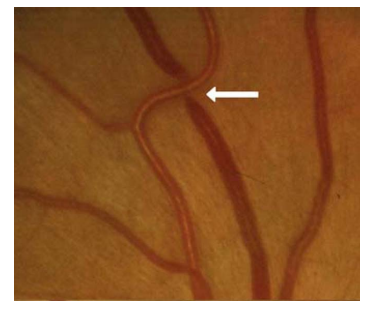

(b)

Figure 2.4: Some retinal vascular signs: (a) Focal arteriolar narrowing and (b) Arteriovenous nicking. Images extracted from (Liew and Wang, 2011).

In general, state-of-the-art software applications that compute measurements of retinal vessels for hypertensive risk determination are focused only on caliber determination and no other measures are performed. At the beginning, software applications were not used to detect retinal vessels or measure geometrical properties automatically but the diameters of all arterioles and venules were measured by trained graders using different tools provided by the systems to make this task simpler (Wong et al., 2004; Knudtson et al., 2003; Hubbard et al., 1999). Nowadays, there are some automatic or semiautomatic approaches to perform these measures (Ruggeri et al., 2007; Muramatsu et al., 2010; Tramontan et al., 2008; Niemeijer et al., 2011; Ortega et al., 2010). 


\subsubsection{Diabetic retinopathy}

Diabetes is a metabolic disease due to insufficient production of the hormone insulin, or a resistance to its action which prevents the glucose uptake of the blood by the cells of the organism giving place to high blood sugar levels. Diabetic retinopathy (DR) is a common symptom of diabetes and one of the leading causes of blindness worldwide. In fact, this disease occurs in $80 \%$ of patients who have had diabetes for more than 10 years (Wang et al., 2000). To ensure that DR treatment is received on time, fundus images from diabetic patients must be examined at least once a year (Fong et al., 2003).

Diabetic retinopathy is a silent disease because only affects vision when retina changes have progressed to a level where treatment is complicated and expensive and the maintenance of vision is uncertain. The most effective treatment of the disease can only be administered in the early stages and, thus, early detection through regular screening is of paramount importance. For automatic screening of DR, most systems are based on the detection of the following features (Yun et al., 2008):

- Microaneurysms. Microaneurysms are the first identifiable characteristic of the disease. They are small saccular dilatations of capillaries that appear as round spots of dark red color.

- Exudates. Exudates are accumulations of lipids and proteins in the retina. They are typically bright lesions of white or cream color.

- Hemorrhages. Hemorrhages become visible when the DR degree progresses. They indicate an ischemia increase (lack of oxygen) of the retina.

DR can be broadly classified as nonproliferative DR (NPDR) and proliferative DR (PDR) depending on the presence and amount of the mentioned features (Early Treatment DR Study Research Group, 1991). 
Figure 2.5 identifies the different types of DR features and Figure 2.6 shows two fundus images with NPDR and PDR, respectively.
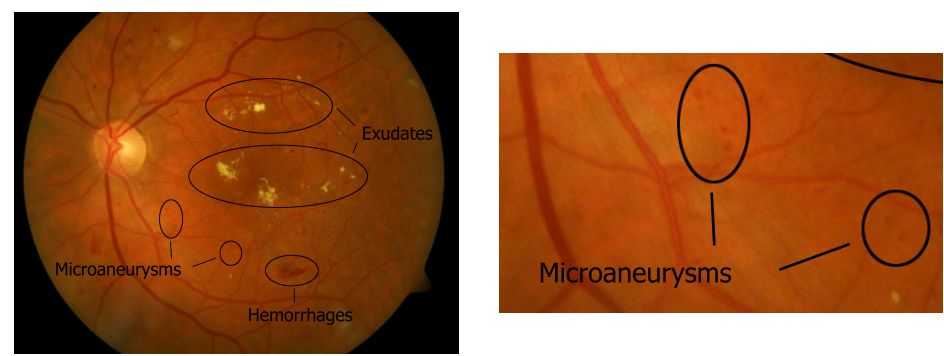

Figure 2.5: Features of diabetic retinopathy (zoom in the area with microaneurysms).

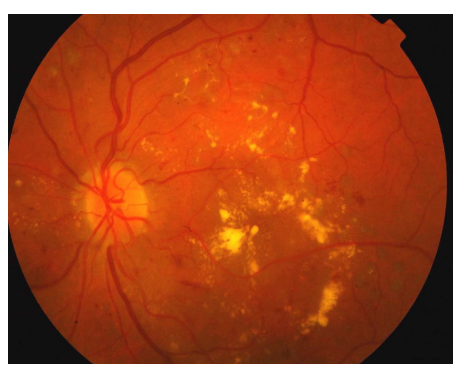

(a)

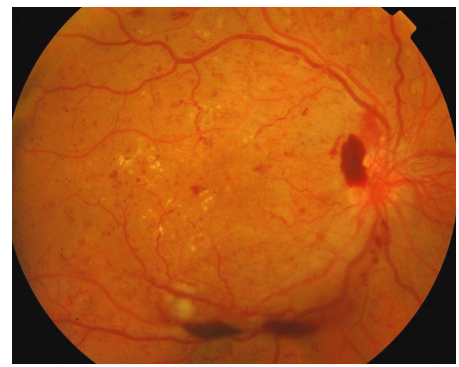

(b)

Figure 2.6: Grades of diabetic retinopathy: (a) Nonproliferative DR (also known as background $D R$ ) and (b) Proliferative DR.

$\mathrm{DR}$ is the disease which more research groups are working on. So, DR and normal retina have been classified for multitude of methods with different results up to now. Some of them will be enumerated. Multilayer perceptron neural network is used in (Sinthanayothin et al., 2002 ) achieving a sensitivity of $80.21 \%$ and a specificity of $70.66 \%$. The identification of exudates, hemorrhages and microaneurysms is needed to carry out the screening in (Singalavanija et al., 2006), which gets a sensitivity of $74.8 \%$ and a specificity of $82.7 \%$. Microaneurysms 
are detected by Bayes optimality criteria and early detection of DR is performed by a decision support system in (Kahai et al., 2006) obtaining a sensibility of $100 \%$ but a specificity of $67 \%$. An automated grading for DR detection with $90.5 \%$ sensitivity and $67.4 \%$ specificity is used in (Philip et al., 2007). The classification is based on fovea, blood vessels, optic disk and bright and dark lesion segmentations in (Estabridis and de Figueiredo, 2007) obtaining 90\% of accuracy. A sensitivity of $84 \%$ and a specificity of $64 \%$ was achieved by the system proposed in (Abràmoff et al., 2008). Note that these works are validated on different databases therefore their direct comparison is difficult. A wider review about DR detection systems can be found in (Faust et al., 2012).

\subsubsection{Age-related macular degeneration}

Age-related macular degeneration (AMD) is a degenerative condition which degrades progressively the macula. The proper functioning of the macula lets appreciate details, reading, driving, face recognizing, etc. AMD is a common eye disease that affects adults and it has become the leading cause of blindness over 60 years (Jager et al., 2008). Its progression is so slow that patients only notice small changes in their vision although it can provoke a total loss of central vision. In spite of the fact that AMD has currently no definitive cure, early detection allows to provide treatments that retard and mitigate the effects of the disease (Jager et al., 2008).

Drusen, yellow deposits in the macula, are one of the early signs more common of the AMD and the basis on which ophthalmologists pay attention to detect the disease. Drusen usually are of two types: hard and soft. Hard drusen are small, round, with sharp edges. These drusen are generally not harmful if they present in small quantity. Soft drusen are larger than the hard drusen and may have indistinct borders. As the disease progresses, the number and size of the drusen increases and other signs can also appear such as geographic atrophy of the retinal pigment epithelium, choroidal neovascularization, disciform scar, etc. 
(Miller, 2013). In Figure 2.7, different signs of AMD can be observed.

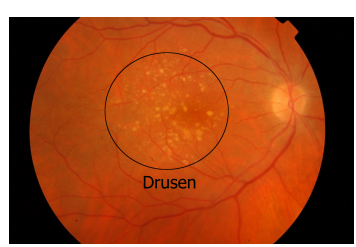

(a)

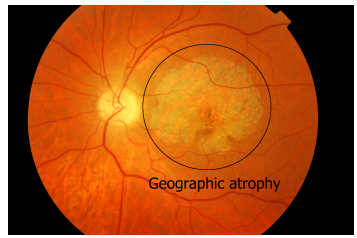

(b)

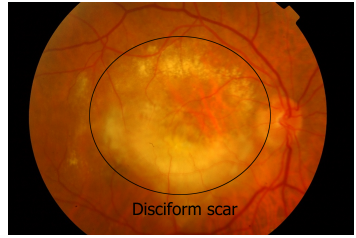

(c)

Figure 2.7: Signs of age-related macular degeneration: (a) Drusen, (b) Geographic atrophy of the retinal pigment epithelium and (c) Disciform scar.

Most works of the state of the art related to AMD is based on drusen segmentation but not in AMD diagnosis (Hijazi et al., 2012) although some reports can be found (Agurto et al., 2011a; Garnier et al., 2014). Agurto et al. use an Amplitude Modulation - Frequency Modulation (AM-FM) technique to define the features followed by two-step Partial Least Squares (PLS) classifier achieving a sensitivity of $94 \%$ and a specificity of $50 \%$. Garnier et al. do not perform drusen segmentation but they analyze texture information through wavelet decomposition and local binary patterns. A sensitivity of $91 \%$ and a specificity of $95 \%$ are obtained using a LDA classifier. These methods are validated on different private databases.

\subsubsection{Glaucoma}

Glaucoma is a progressive disease of the optic nerve caused by high intraocular pressure due to a bad drainage of the ocular fluid. Clinically, it causes a progressive and irreversible loss of the visual field that progresses to the complete loss of vision. It is currently the second leading cause of blindness worldwide and affects one in every hundred people under 50 and one in ten over 80 years (Kwon et al., 2009). In most cases, glaucoma is belatedly detected when visual field loss is irreversible. Currently, there is no cure for glaucoma damage and, 
therefore, early detection and prevention is the only way to prevent progression to total vision loss.

According to the literature, the diagnosis of glaucoma is mainly based on three studies: the measurement of intraocular pressure by tonometry, the examination of the visual field by campimetry and the computing of the ratio between the optic cup and optic disk area from fundus images, also known as cup-to-disk ratio (CDR) (Nath and Dandapat, 2012). Since this thesis focuses on the detection of glaucoma by means of retinal image analysis, it is the last of the studies, the measure of the cup-disk ratio, which is of our interest. The optic nerve damage is manifested in a change in the appearance of the optic cup, so that its size (and therefore the CDR) is related to the presence or absence of glaucoma. For healthy patients, CDR falls in the range of 0.3 to 0.5 and for glaucoma it is greater than 0.5. The sight of vision is completely lost at the CDR value of 0.8 (Nath and Dandapat, 2012). In Figure 2.8 the existing difference between a fundus with and without glaucoma can be appreciated.

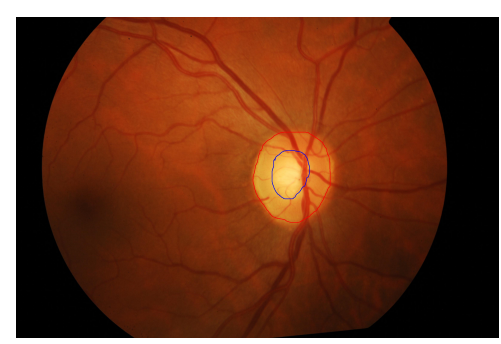

(a)

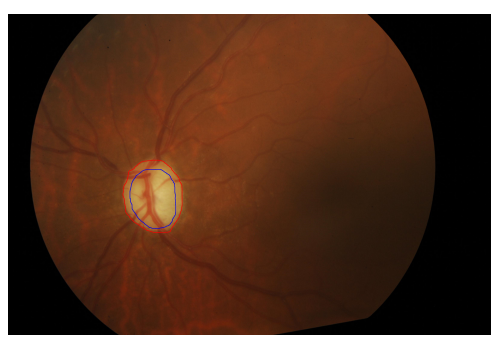

(b)

Figure 2.8: Glaucoma effects on the cup-to-disk ratio: (a) Healthy fundus and (b) Glaucomatous fundus. Optic disk is marked in red and optic cup in blue.

Among the different works that deal with glaucoma detection the following can be stood out. The ARGALI system performs an automatic cup-to-disk ratio measurement using level-sets and achieves a RMS error 
of 0.05 with a risk assessment accuracy of $95 \%$ (Liu et al., 2009). Nayak et al. not only use the CDR as feature but also take into account other anatomical ratios (the ratio of the distance between optic disk center and optic nerve head to diameter of the optic disk and the ratio of blood vessels area in inferior-superior side to area of blood vessels in the nasaltemporal side). All these features are incorporated in a neural network classifier which obtains a sensitivity and specificity of $100 \%$ and $80 \%$ respectively (Nayak et al., 2009). Hatanaka et al. carried out a different approach and measure the cup-to-disk ratio through the vertical profile of the optic disk getting a sensitivity of $80 \%$ and a specificity of $85 \%$ (Hatanaka et al., 2010). These statistics were computed on different databases. More techniques of glaucoma detection can be found in (Nath and Dandapat, 2012).

\subsection{Relevant groups}

The most relevant groups working on retinal image processing, which have developed some prototype of detection systems of one or more pathologies under study in this thesis, are:

\section{International groups}

The Retinal Image Computing \& Understanding group ${ }^{3}$ at the University of Lincoln (United Kingdom) works on segmentation and measurement of the retinal vessels (Lowell et al., 2004; Al-Diri et al., 2009; Al-Diri and Hunter, 2009) and optic disk segmentation (Lowell et al., 2004). Their works are mainly related to hypertension although some publications are referred to diabetic retinopathy (Massey et al., 2009). Nowadays, they are the coordinators of a European project, named "REVAMMAD - REtinal Vascular Modelling, Measurement and Diagnosis" 4 , included in the Seventh Framework Programme.

\footnotetext{
${ }^{3}$ www.aldiri.info/Default.aspx

${ }^{4}$ http://revammad.blogs.lincoln.ac.uk/revammad/
} 
VisionQuest Biomedical ${ }^{5}$ (Albuquerque) focuses on diabetic retinopathy screening although they also work on the detection of other pathologies such as age-related macular degeneration (Agurto et al., 2011a; Agurto et al., 2011b). Their system EYESTAR ${ }^{6}$ is now in a clinical trial.

The Institute for Infocomm Research ${ }^{7}$ (Singapore). They have mainly worked on glaucoma detection through a system for automatic measurement of the cup-to-disk ratio called ARGALI (Liu et al., 2009). They have also worked, along with the National University of Singapore, on the development of a platform for vascular disease risk prediction (Hsu et al., 2011).

The Computer Vision and Image Processing (CVIP) group 8 at the University of Dundee (Scotland) has worked on the implementation of VAMPIRE ${ }^{9}$, a vessel assessment and measurement platform (PerezRovira et al., 2011).

The University of Aberdeen (Scotland) has worked on an automated grading of diabetic retinopathy in primary care screening (Philip et al., 2007; Goatman et al., 2011). ${ }^{10}$ Although only for a disease, it is the only existing CE-accredited commercial system in terms of analysis for automatic fundus screening. ${ }^{11}$

\footnotetext{
${ }^{5}$ www.visionquest-bio.com

${ }^{6}$ www.visionquest-bio.com/eyestar-tm.html

${ }^{7}$ www.i2r.a-star.edu.sg/industries/medical-and-healthcare

${ }^{8}$ http://cvip. computing. dundee.ac.uk/

${ }^{9}$ http://vampire.computing.dundee.ac.uk/

${ }^{10}$ www.abdn.ac.uk/heru/research/assessment-of-technologies/projects/t he-role-of-automated-grading-of-diabetic-retinopa/

${ }^{11}$ ww. shil.co.uk/Products/igradingtm-platform-diabetic-retinopathy-s creening-software.html
} 


\section{National groups}

The VARPA group ${ }^{12}$ at University of A Coruña works mainly on hypertension detection by means of automatic computation of the arteriolar-to-venular ratio. They developed a web application for this purpose known as SIRIUS (Ortega et al., 2010).

The University of Huelva works on an expert system for early automated detection of diabetic retinopathy. ${ }^{13}$ At this moment, there are not results of this system but some algorithms for retinal structure segmentation have been published (Aquino et al., 2010; Marín et al., 2011).

In spite of the amount of groups working on retinal image processing, there is no system that detects at the same time the most common diseases that cause retinal damage. Moreover, the screening systems developed up to now are characterized by achieving high sensitivity (correct detection of pathological retinas) but not specificity enough (correct detection of healthy subjects) to be effective for screening purposes. All these facts stand out the need to continue working on this topic.

\footnotetext{
12 www . varpa.es

13 www. uhu.es/retinopathy/index.php
} 



\section{Chapter 3}

\section{Retinal structure characterization}

The retinal vessels and optic disk are two of the most important anatomical structures of the fundus. This chapter presents new methods to detect them and to determine some of their main characteristics. The detection and analysis of these elements are used for hypertensive risk determination. Finally, results and conclusions of the chapter are detailed.

In this chapter, works previously published, although adapted for this thesis, are included:

- Subsection 3.3.1 - Vessel extraction.

Morales, S., Naranjo, V., Angulo, J., Fuertes, J. J., Alcañiz, M. (2012). Segmentation and Analysis of Retinal Vascular Tree from Fundus Images Processing. In International Conference on Bioinspired Systems and Signal Processing (BIOSIGNALS 2012), pages 321-324. 
- Subsection 3.3.2 - Centerline extraction.

Morales, S., Naranjo, V., Angulo, J., López-Mir, F., Alcañiz, M. (2014). Determination of Retinal Network Skeleton through Mathematical Morphology. In 22nd European Signal Processing Conference (EUSIPCO 2014), pages 1691-1695.

- Section 3.4 - Significant points.

Morales, S., Naranjo, V., Colomer, A., Alcañiz, M. (2015). Significant point characterization in fundus images. In 5th International Conference on Image Processing Theory, Tools and Applications (IPTA 2015) (Accepted for publication).

- Section 3.5 - Optic disk segmentation.

Morales, S., Naranjo, V., Angulo, J., Alcañiz, M. (2013). Automatic detection of optic disc based on PCA and mathematical morphology. IEEE Transactions on Medical Imaging, 32(4): 786796 .

- Section 3.6 - Application to pathology detection: Hypertensive risk.

Morales, S., Naranjo, V., Navea, A., Alcañiz, M. (2014). Computer-Aided Diagnosis Software for Hypertensive Risk Determination Through Fundus Image Processing. IEEE Journal of Biomedical and Health Informatics, 18(6):1757-1763. 


\section{Contents}

3.1 Introduction $\ldots \ldots \ldots \ldots \ldots \ldots \ldots$

3.2 Theoretical background . . . . . . . . . 34

3.2 .1 Morphological operators . . . . . . . . . . . 34

3.2.2 Stochastic watershed transformation . . . . . 39

3.3 Vessel segmentation . . . . . . . . . . . . . 43

3.3.1 Vessel extraction . . . . . . . . . . . . . . . . . . 45

3.3 .2 Centerline extraction . . . . . . . . . . . 50

3.4 Significant points . . . . . . . . . . 56

3.4.1 Terminal and bifurcation points . . . . . . . 58

3.4.2 Crossing points . . . . . . . . . . . . . . . . 60

3.5 Optic disk segmentation $\ldots \ldots \ldots \ldots$

3.5.1 Pre-processing . . . . . . . . . . . . . . 66

3.5 .2 Processing. . . . . . . . . . . . . 71

3.5.3 Post-processing . . . . . . . . . . 78

3.6 Application to pathology detection: Hypertensive risk ............. 79

3.6 .1 Pre-processing . . . . . . . . . . . . . . . . . . . . 81

3.6 .2 Processing . . . . . . . . . . . . . . . . . . 82

3.6 .3 Post-processing . . . . . . . . . . . . 83

3.7 Results . . . . . . . . . . . 86

3.7 .1 Material . . . . . . . . . . . . . . . 86

3.7 .2 Retinal vessel segmentation . . . . . . . . . . 89

3.7.3 Retinal vessel centerline . . . . . . . . . . . . 94

3.7.4 Optic disk . . . . . . . . . . . . . . . . 95

3.7.5 Computer-aided diagnosis software for hypertensive risk determination . . . . . . . . . 112

3.8 Conclusions . . . . . . . . . . . . . 115 



\subsection{Introduction}

Retinal structure segmentation is a fundamental component of most automatic retinal disease screening systems (Fraz et al., 2012). It is usually a prerequisite before systems carrying out more complex tasks as identifying different pathologies. In general, several anatomical structures are segmented through fundus image processing and then some features are extracted from them to characterize each pathology. The most important anatomical structures of the fundus are the vascular network that is the retinal blood vessels, and the optic disk (OD). Both of them can be used as reference to detect other anatomical ocular structures or several retinal lesions and to identify some fundus features. For example, among their multiples uses, the following can be stressed. Morphological attributes of retinal blood vessels, such as length, width, tortuosity and/or branching pattern and angles are utilized for diagnosis, screening, treatment, and evaluation of various cardiovascular and ophthalmologic diseases (Kanski and Bowling, 2011). The relation between the size of the optic disk and the cup (cup-to-disk ratio) is widely used for glaucoma diagnosis (Hatanaka et al., 2010). The relatively constant distance between the optic disk and the fovea is useful for estimating the location of the macula, area of the retina related to fine vision (Niemeijer et al., 2009). Their detection may also be used for not taking them into account in the analysis of the retina background with the aim of pathology diagnosis, as discussed in Chapter 4. Due to their importance in screening systems of ophthalmologic diseases, this chapter presents new algorithms related to detection and characterization of the retinal vessels and the optic disk. Their performance and their place with respect to previous studies will be detailed along the chapter.

Numerous segmentation methods of retinal structures have been reported in the literature. Nevertheless, only a review of the state of the art of the methods regarding the description of the vascular tree and the optic disk will be performed here. 
Referring to vessel extraction techniques, they can be mainly divided into four categories: edge detectors, matched filters, pattern recognition techniques and morphological approaches. A more extensive classification can be found in (Fraz et al., 2012). Most edge detection algorithms assess changes between pixels values by calculating image gradient magnitude and then it is thresholded to create a binary edge image (Martinez-Perez et al., 2007; Jiang and Mojon, 2003). Matched filters are filters rotated in different directions in order to identify the cross section of blood vessels (Hoover et al., 2000; AlRawi et al., 2007). Pattern recognition techniques can be divided into supervised and unsupervised approaches. Supervised methods, such as artificial neural networks (Sinthanayothin et al., 1999) or support vector machines (Ricci and Perfetti, 2007; Marín et al., 2011), exploit some prior labelling information to decide whether a pixel belongs to a vessel or not, while unsupervised algorithms perform the vessel segmentation without any prior labelling knowledge (Kande et al., 2010). Morphological processing is based on vessels characteristics known a priori (line connected segments) and combines morphological operators to achieve the segmentation (Zana and Klein, 2001; Mendonça and Campilho, 2006; Sun et al., 2011). Although most of the state-ofthe-art methods look for detecting all vessel pixels of the vascular tree, there are also some attempts based on finding the vessel skeleton or, in other words, the vessel centerline. The work of Chen et al. is based on shortest path connection (Chen et al., 2011), Sofka and Stewart on the use of matched filters (Sofka and Stewart, 2006), Wu and Derwent on ridge descriptors (Wu et al., 2009) and Walter and Klein and Bessaid et $a l$. on the application of watershed transformation (Walter and Klein, 2002; Bessaid et al., 2009).

Once the vessels are segmented, the identification of the vascular bifurcations and crossovers is helpful for predicting cardiovascular diseases and can be used as biometric features and for image registration (Grisan et al., 2004; Bevilacqua et al., 2005; Bhuiyan et al., 2007; Calvo et al., 2011; Azzopardi and Petkov, 2011). After identifying the vascular bifurcations, the angles generated in these points are usually measured 
by fitting the vessels that form the bifurcation by straight lines and then estimating the angle between them (Gao et al., 2000; Martinez-Perez et al., 2002; MacGillivray et al., 2012).

Regarding the OD-boundary detectors, the presented techniques can mainly be grouped into template-based methods, deformable models, and morphological algorithms. Different approaches have been proposed according to template-based methods: edge detection techniques followed by the Circular Hough Transform (Park et al., 2006; Aquino et al., 2010); pyramidal decomposition and Hausdorffbased template matching (Lalonde et al., 2001); color decorralated templates (Kauppi and Kälviäinen, 2008); or a kNN-regressor along with a circular template (Niemeijer et al., 2009). Concerning deformable models, GVF-snake (Osareh et al., 2002), ASM (Li and Chutatape, 2004) and modified active contours, which exploit specific features of the optic disk anatomy (Lowell et al., 2004) or incorporate knowledgebased clustering and updating (Xu et al., 2007), have also been used to this purpose. As for algorithms based on mathematical morphology, most of them detect the OD by means of watershed transformation, generally through marker-controlled watershed (Walter et al., 2002; Eswaran et al., 2008; Welfer et al., 2010), although each author chooses different markers. The centroid of the largest and brightest object of the image is considered as an approximation for the locus of the OD and it is used as internal marker (Walter et al., 2002). The extended minima transformation (Gonzalez et al., 2004) is applied to select the internal markers and external markers are calculated as an effectively partition of the image into regions, so that each region contains single internal marker and part of the background (Eswaran et al., 2008). A list of pixels belonging to the main vessels arcade in the vicinity of an internal OD point previously detected are also used (Welfer et al., 2010). On the other hand, some authors propose combining various algorithms to get a better approximation of the OD-boundary (Hajer et al., 2008; Qureshi et al., 2012).

The rest of the chapter is organized as follows: Section 3.2 
describes the theoretical background. Sections 3.3, 3.4 and 3.5 present the algorithms proposed for detection and characterization of the main retinal structures. Section 3.6 shows how these methods can be applied for hypertensive risk determination. Section 3.7 details the experimental results of the methods explained above and their comparison with other state-of-the-art algorithms. Finally, Section 3.8 provides conclusions.

\subsection{Theoretical background}

This section focuses on introducing the main concepts that will be used in the chapter in order to facilitate its comprehension to the reader. The main morphological operators and the stochastic watershed transformation will be described below to be able to understand the methods developed. More complete readings about mathematical morphology applied to digital image processing can be found in (Serra, 1982; Soille, 2003).

\subsubsection{Morphological operators}

Mathematical morphology is a non-linear image processing methodology based on minimum and maximum operations whose aim is to filter or extract relevant structures of an image (Serra, 1982). Next, different morphological filters will be defined and an explanation about geodesic transformations will also be included. The different operators will be illustrated with figures along the section.

\section{Morphological filters}

Let $f$ be a gray-scale image which is defined as $f(\mathbf{x}): E \rightarrow T$ where $\mathbf{x}$ is the pixel position. In the case of discrete-valued images, $T=\left\{t_{\min }, t_{\min }+1, \ldots, t_{\max }\right\}$ is an ordered set of gray-levels. Typically, in digital 8-bit images $t_{\min }=0$ and $t_{\max }=255$. Furthermore, let $B(\mathbf{x})$ 
be a sub-set of $Z^{2}$ called structuring element (SE) centered at point $\mathbf{x}$, whose shape is usually chosen according to some a priori knowledge about the geometry of the relevant and irrelevant image structures. The two basic morphological operators are:

$$
\begin{aligned}
& \text { Dilation: }\left[\delta_{B}(f)\right](\mathbf{x})=\max _{b \in B(\mathbf{x})} f(\mathbf{x}+\mathbf{b}) \\
& \text { Erosion: }\left[\varepsilon_{B}(f)\right](\mathbf{x})=\min _{b \in B(\mathbf{x})} f(\mathbf{x}+\mathbf{b}) .
\end{aligned}
$$

Their purpose is to expand light or dark regions, respectively, according to the size and shape of the structuring element. See Figure 3.1.

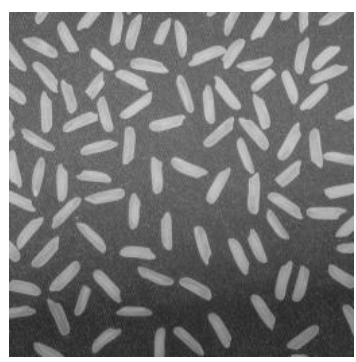

(a)

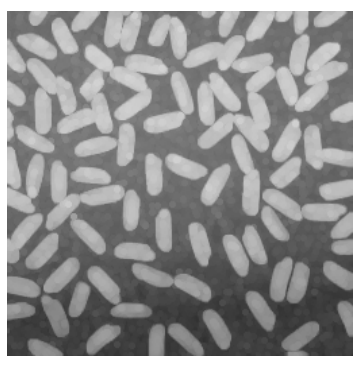

(b)

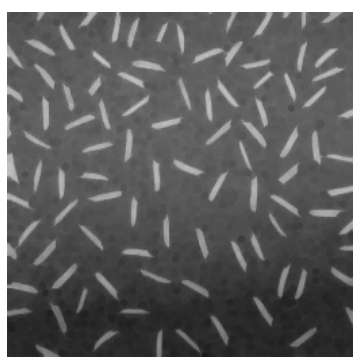

(c)

Figure 3.1: Basic morphological operators: (a) Original image, (b) Dilation and (c) Erosion. Both operators were performed using a disk-shaped structuring element of radius 2.

In mathematical morphology, the gradient $\varrho(f)(\mathbf{x})$ of an image $f(\mathbf{x})$ can be obtained as the pointwise difference between a unitary dilation and a unitary erosion, i.e.,

$$
\varrho(f)(\mathbf{x})=\delta_{B}(f)(\mathbf{x})-\varepsilon_{B}(f)(\mathbf{x}) .
$$

The goal of the gradient transformation is to highlight image contours. 
Dilation and erosion can be combined successively to obtain a new set of operators or basic filters given by:

$$
\begin{aligned}
& \text { Opening: } \gamma_{B}(f)=\delta_{B}\left(\varepsilon_{B}(f)\right) \\
& \text { Closing: } \varphi_{B}(f)=\varepsilon_{B}\left(\delta_{B}(f)\right) .
\end{aligned}
$$

Light or dark structures are respectively filtered out from the image by these operators regarding the structuring element chosen (Figure 3.2).

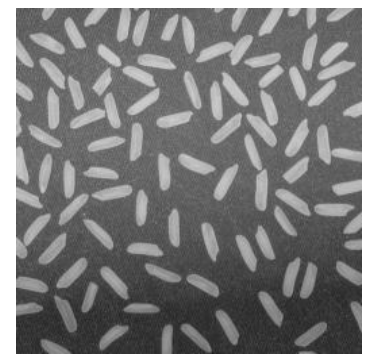

(a)

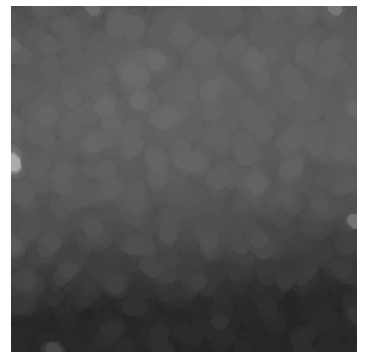

(b)

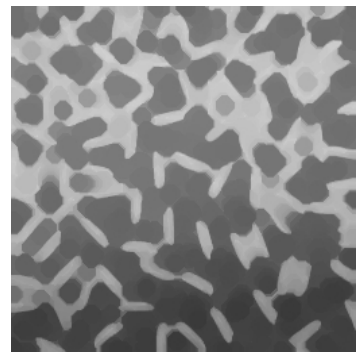

(c)

Figure 3.2: Basic morphological filters: (a) Original image, (b) Opening and (c) Closing. Both filters were performed using a disk-shaped structuring element of radius 5 .

Openings and closings act as non-linear low-pass filters simplifying the details of an image. However, the objects of interest may also be those details and, not always, only one operator is enough to obtain all the desired objects. To solve these problems there exist other morphological operators derived from the previous ones: top-hats and the supremum of openings or the infimum of closings.

The top-hat transformation aims to suppress slow trends, therefore to enhance the contrast of some features in images, according to size or shape criteria. The top-hat (or positive top-hat) is the residue between the original image and its opening and the dual top-hat (or negative top-hat) can be defined as the residue between a closing and its original 
image, i.e.,

$$
\begin{gathered}
\text { Top-hat: } \rho_{B}^{+}(f)=f-\gamma_{B}(f) \\
\text { Dual top-hat: } \rho_{B}^{-}(f)=\varphi_{B}(f)-f
\end{gathered}
$$

The top-hat transformation is used to extract contrasted components with respect to the background (Figure 3.3(c)). The basic top-hat extracts positive components and the dual top-hat the negative ones.

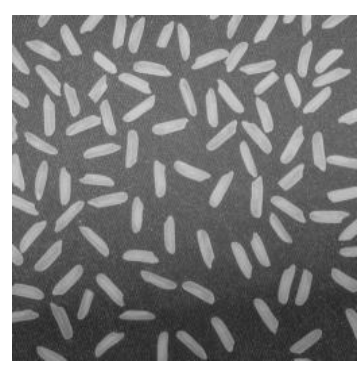

(a)

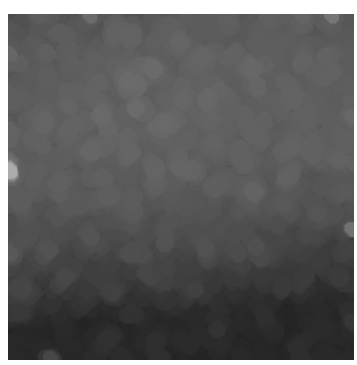

(b)

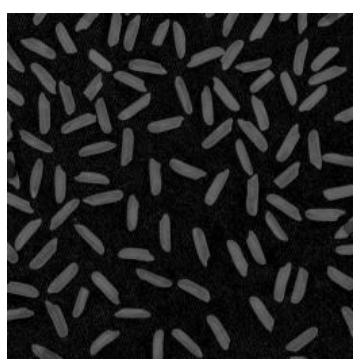

(c)

Figure 3.3: Top-hat transformation: (a) Original image, (b) Opening with a circular SE of radius 5 and (c) Top-hat.

For a collection $\gamma_{i}$ of openings, the supremum of openings, $\gamma^{\text {sup }}=$ $\bigvee_{i} \gamma_{i}$ can be considered as the union of all openings which is also an opening. Figure 3.4 shows an example where the supremum of two openings performed with linear SE of different orientations was performed. This is useful when the object to be extracted has not a unique orientation, as in the case of the figure. On the other hand, if $\varphi_{i}$ is a collection of closings, then the infimum of closings, $\varphi^{i n f}=\bigwedge_{i} \varphi_{i}$ is the intersection of all closings which is a closing as well. 


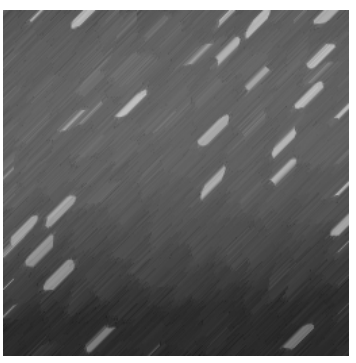

(a)

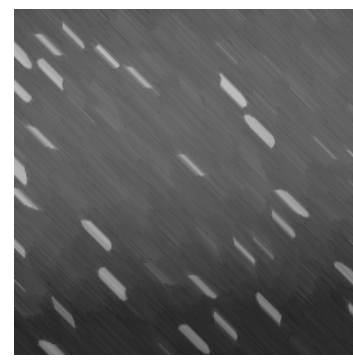

(b)

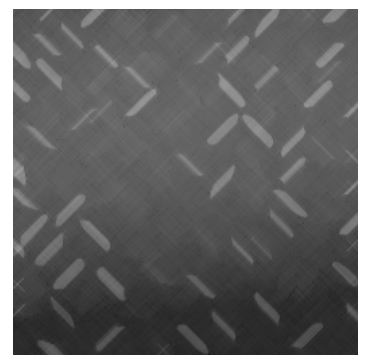

(c)

Figure 3.4: Supremum of openings: (a) Opening with a linear $S E\left(+45^{\circ}\right)$, (b) Opening with a linear $S E\left(-45^{\circ}\right)$ and (c) Supremum of linear openings. The original image is that shown in Figure 3.3(a)

\section{Geodesic operators}

Other operators used in this thesis are: reconstruction by dilation and close-holes. First, elementary geodesic transformations will be introduced to be able to explain these operators. The geodesic transformations involve two images: a marker image $f$ and a reference image $g$. By definition, the reference image must be greater than or equal to the marker image $(f \leq g)$.

The geodesic dilation is the iterative unitary dilation of the marker image $f$ with respect to the reference image $g$,

$$
\delta_{g}^{(n)}(f)=\delta_{g}^{(1)} \delta_{g}^{(n-1)}(f) \text {, being } \delta_{g}^{(1)}(f)=\delta_{B}(f) \wedge g
$$

The reconstruction by dilation is the successive geodesic dilation of $f$ regarding $g$ up to idempotence,

$$
R_{g}^{\delta}(f)=\delta_{g}^{(i)}(f), \text { so that } \delta_{g}^{(i)}(f)=\delta_{g}^{(i+1)}(f)
$$

The reconstruction by erosion can be obtained as its dual operator, 
$R_{g}^{\varepsilon}(f)=\left[R_{g^{c}}^{\delta}\left(f^{c}\right)\right]^{c}$, being $f^{c}$ the complement image (i.e., the negative). The reconstruction by dilation removes from the reference image $g$ the light objects unconnected with the marker image $f$ and the reconstruction by erosion the dark ones (Figure 3.5).

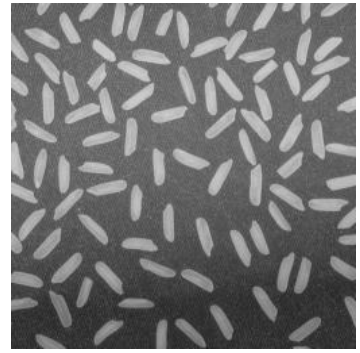

(a)

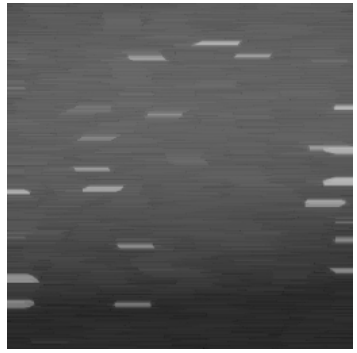

(b)

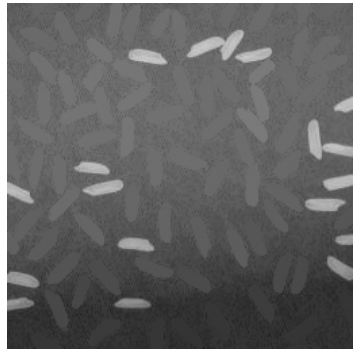

(c)

Figure 3.5: Geodesic reconstruction: (a) Reference image, (b) Marker image and (c) Reconstruction by dilation.

Using the reconstruction by dilation, the close-hole operator can also be defined. For a gray-scale image, it is considered a hole any set of connected points surrounded by connected components of value strictly greater than the hole values. This operator fills all holes in an image $f$ that do not touch the image boundary $f_{\partial}$ that is used as marker (Figure $3.6)$ :

$$
\psi^{c h}(f)=\left[R_{f^{c}}^{\delta}\left(f_{\partial}\right)\right]^{c}
$$

\subsubsection{Stochastic watershed transformation}

Watershed transformation is a segmentation technique for gray-scale images (Beucher and Meyer, 1992). This algorithm is a powerful segmentation tool whenever the minima of the image represent the objects of interest and the maxima are the separation boundaries between objects. Due to this fact, the input image of this method 


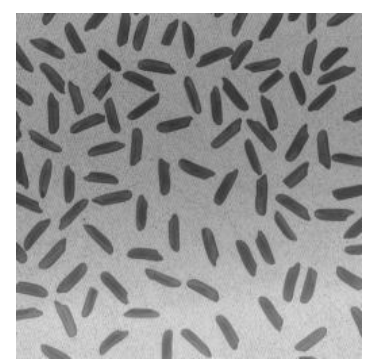

(a)

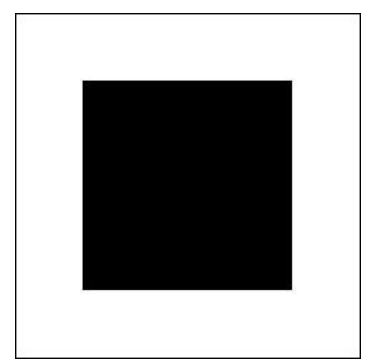

(b)

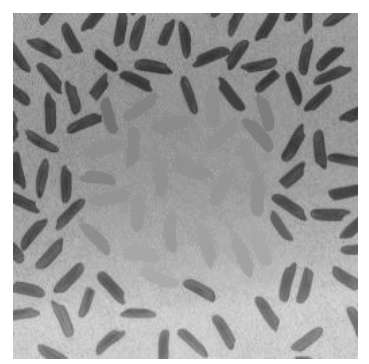

(c)

Figure 3.6: Close-hole operator: (a) Reference image, (b) Marker image and (c) Close-hole.

is usually a gradient image $\varrho(f)(\mathbf{x})$. The watershed transformation produces a segmentation which can be viewed as: a set of closed contours of segmented regions which will be noted by $W S(\varrho(f))$, or a partition of the space $\mathrm{E}$ in a set of classes named $\Pi(W S(\varrho(f)))$.

One problem of this technique is the over-segmentation, which is caused by the existence of numerous local minima in the image normally due to the presence of noise. One solution to this problem is using marker-controlled watershed, $W S(\varrho)_{f_{m r k}}$, in which the markers $f_{m r k}$ artificially indicate the minima of the image. Nevertheless the controversial issue consists in determining $f_{m r k}$ for each region of interest,

$$
f_{m r k}(\mathbf{x})= \begin{cases}0 & \text { if } \mathbf{x} \in \text { marker } \\ 255 & \text { Otherwise }\end{cases}
$$

Note that the number of markers determines the number of regions obtained. The use of few markers along with the existence of borders within the structure to be segmented can also cause that some parts of it are not detected (under-segmentation). So that, the choice of the correct markers is crucial for the effectiveness and robustness of the 
algorithm. In marker definition not only markers contained inside the objects of interest (internal markers) are needed but also a marker that determines the image background (external marker). For example, in the case that only a single region was desired, two markers should be used, one internal and other external. Figure 3.50 shows the over and under-segmentation problems related to the watershed transformation when attempting to segment the optic disk.

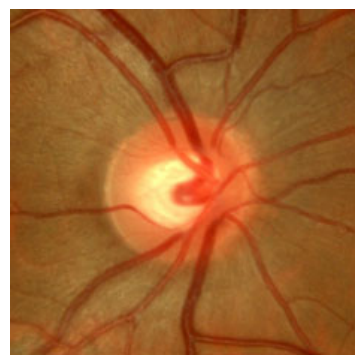

(a)

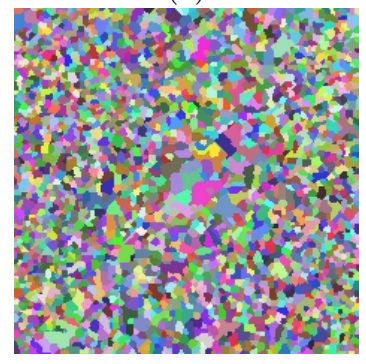

(d)

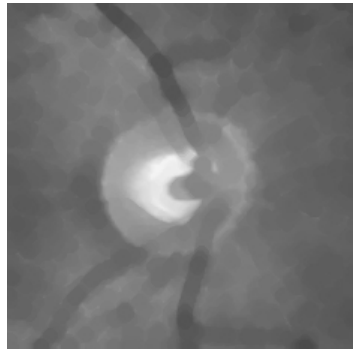

(b)

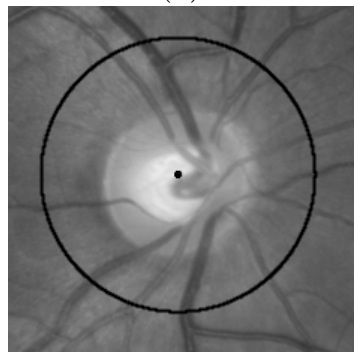

(e)

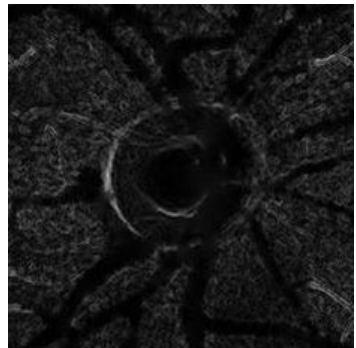

(c)

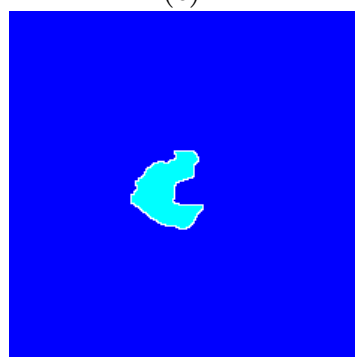

(f)

Figure 3.7: $\quad$ Over and under-segmentation problems of the watershed transformation: (a) Original image, (b) Gray image after closing operator for vessel removal, (c) Gradient image, (d) Classical watershed (oversegmentation), (e) Artificial markers and (f) Marker-controlled watershed using the artificial markers (under-segmentation).

There exist different strategies to choose the markers to be used in the marker-controlled watershed. The following ones can be stood out: 
- Markers based on a seed. This seed can be chosen manually or selected automatically according to the significance of its intensity level, for instance the minima having a local dynamics larger than a given value (Beucher and Meyer, 1992). Another example can be the pixel with the highest/lowest intensity value (Walter et al., 2002).

- Stratified markers. Stratified markers are uniform markers generated within an area. The generation of stratified markers consists of dividing the region to be segmented into a uniform grid, and only the centroid of each region is considered as marker (Chilès and Delfiner, 2012; Bernander et al., 2013).

- Uniform random markers. Random markers are uniformly distributed in the image domain to be segmented (Angulo and Jeulin, 2007).

- Regionalized random markers. Regionalized random markers are non-uniform random markers whose distribution is restricted to areas that accomplished a specific condition, for example they can only be located in high-intensity areas (Angulo and Jeulin, 2007; Angulo and Velasco-Forero, 2010).

In this thesis, stratified and uniform random markers are used. It can be observed in Subsection 3.3.2 and Section 3.5.

Making use of random markers, a watershed transformation variant is used to solve over and under-segmentation problems related to the classical watershed, the stochastic watershed (Angulo and Jeulin, 2007). In this transformation, a given number $M$ of marker-controlledwatershed realizations are performed selecting $N$ random markers in order to estimate a probability density function $(p d f)$ of image contours and filter out non-significant fluctuations. Let $\left\{f_{m r k i}\right\}_{i=1}^{M}$ be $M$ sets of $N$ uniform random markers and $W S_{i}=W S(\varrho)_{f_{m r k} i}$ the $i$ th output image of the marker-controlled watershed imposed by $f_{m r k i}$. The $p d f$ 
of image contours is computed by Parzen window method (Duda and Hart, 1973) as follows

$$
p d f(\mathbf{x})=\frac{1}{M} \sum_{i=1}^{M}\left(W S_{i}(\mathbf{x}) * G(\mathbf{x} ; s)\right)
$$

where $G(\mathbf{x} ; s)$ represents a Gaussian kernel of variance $\sigma^{2}$ and mean $\mu$ $(\mu=0)$

$$
G(\mathbf{x} ; s)=\frac{1}{2 \pi \sigma^{2}} e^{-\left(\frac{\|\mathbf{x}\|^{2}}{2 \sigma^{2}}\right)} .
$$

Figure 3.8 illustrates how the $p d f$ is generated. Afterwards, it is necessary to perform a last marker-controlled watershed on the $p d f$ obtained.

This type of watershed works better than other marker-based watershed transformations used previously in the literature. In the next sections, two different applications of the stochastic watershed will be explained in detail, one for vessel centerline extraction and other for optic disk segmentation.

\subsection{Vessel segmentation}

Retinal vasculature is able to indicate the status of other vessels of the human body. Indeed, the retina is the only location where blood vessels can be directly visualized non-invasively in vivo (Patton et al., 2006). That is the reason why its study is usually included in the standard screening of any patient with diseases in which the vessels may be altered. In many instances, preclinical signs are not easily recognized and often appear as signs or symptoms that are not specific for a particular disease, but the retina and its blood vessel characteristics 


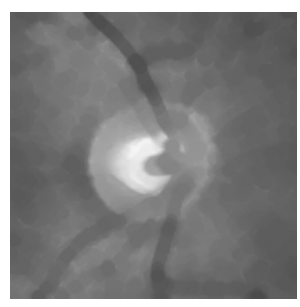

(a)

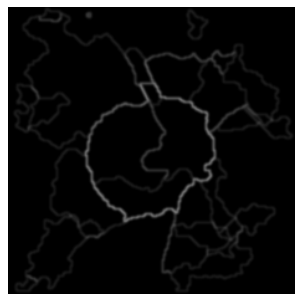

(b)

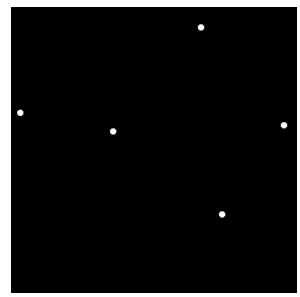

(c)

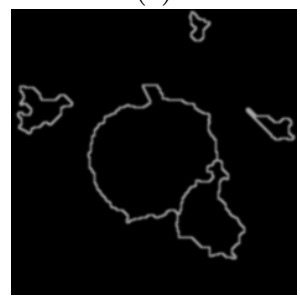

(f)

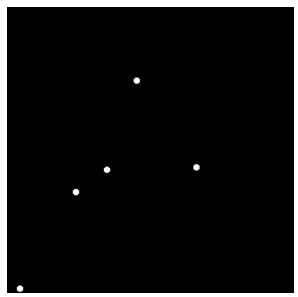

(d)

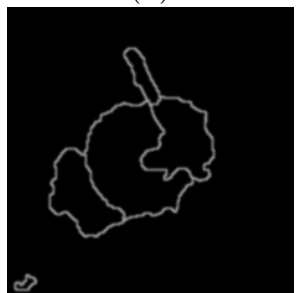

(g)

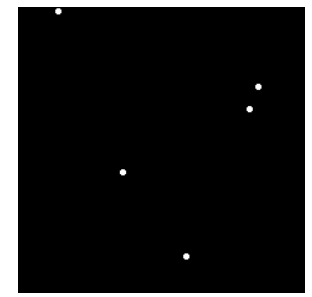

(e)

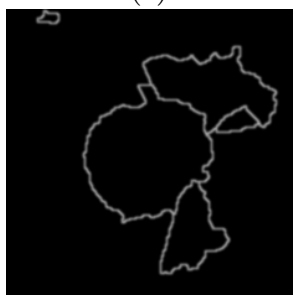

(h)

Figure 3.8: Probability density function: (a) Original image f, (b) Probability density function of contours computed by Parzen window method for $N=5$ and $M=5$, (c-e) Random markers $f_{m r k_{i}}$ and $(f-h)$ Corresponding markercontrolled-watershed realizations $W S_{i}$. The pdf is generated by combining the different $W S_{i}$.

have shown to be a window into several disease processes (Cree and Jelinek, 2011).

In this section, algorithms for vascular network determination will be presented, both for extraction of the complete retinal vasculature and the vessel centerline. 


\subsubsection{Vessel extraction}

The proposed segmentation method is based on mathematical morphology and curvature evaluation for the detection of retinal vascular tree. Although fundus images are RGB images, only monochrome images obtained from the green band are drawn on because they provide an improved visibility of retinal blood vessels as shown in Figure 3.9 .

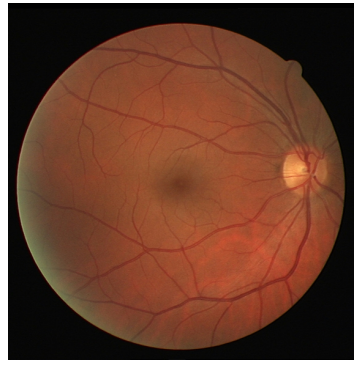

(a)

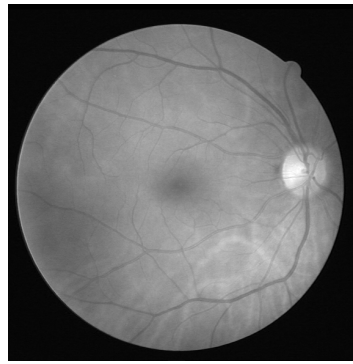

(b)

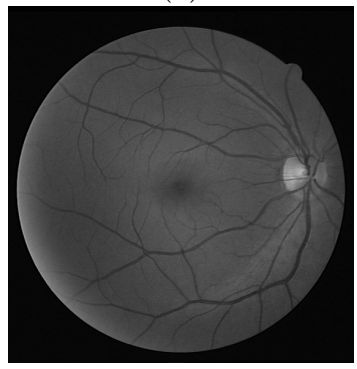

(c)

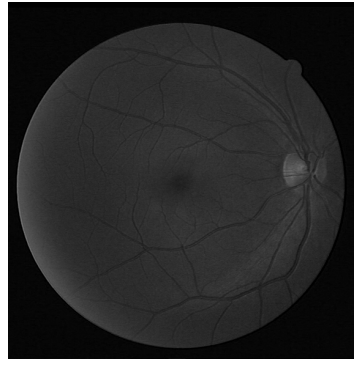

(d)

Figure 3.9: $R G B$ to gray conversion: (a) Original RGB image, (b) Red component, (c) Green component and (d) Blue component.

Non-uniform illumination and low contrast are typical and inherent problems to the image capture technique. Moreover, in the case of retinal images, both problems are especially pronounced due to the fact that the retina is a spherical structure and it is necessary the use of a spotlight to capture the image. So, they should be corrected previously 
to the image processing. In this work, next local transformation for shade correction (Walter and Klein, 2002) was used:

$$
\Gamma(f)(t)= \begin{cases}\frac{\frac{1}{2}\left(u_{\max }-u_{\min }\right)}{\left(\mu_{f}-t_{\min }\right)^{r}}\left(t-t_{\min }\right)^{r}+u_{\min } & \text { if } t \leq \mu_{f} \\ \frac{-\frac{1}{2}\left(u_{\max }-u_{\min }\right)}{\left(\mu_{f}-t_{\max }\right)^{r}}\left(t-t_{\max }\right)^{r}+u_{\max } & \text { if } t>\mu_{f},\end{cases}
$$

where $t_{\min }$ and $t_{\max }$ are the minimum and maximum gray level of the image respectively, $u_{\min }$ and $u_{\max }$ are the target levels (typically 0 and 255 respectively), $\mu_{f}$ is the mean value of the image for all pixels within a window centered at the current pixel $\mathbf{x}$ of the fixed size $L$, and the parameter $r$ is used to control the contrast increasing (experimentally $L=51$ and $r=3$ for fundus images). The effect of this transformation can be observed in Figure 3.10. Note that all size parameters used in the vessel extraction method are optimized for the resolution of the images belong to DRIVE database (Staal et al., 2004), which will be described in the material subsection (Subsection 3.7.1).

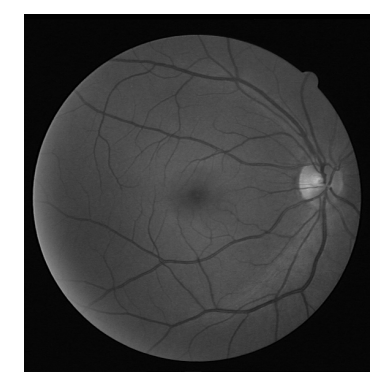

(a)

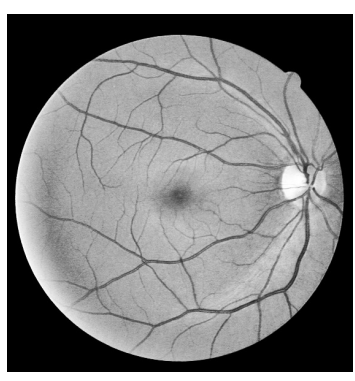

(b)

Figure 3.10: Image enhancement: (a) Green component and (b) Local shade correction.

After image pre-processing, the following stages are implemented for vessel segmentation: 
First, a small opening, using a disk of radius $1\left(B_{1}\right)$ as structuring element (SE), is performed on the enhanced green component image to fill in any gaps of the vessels which could induce errors in segmentation. See Figure 3.11.

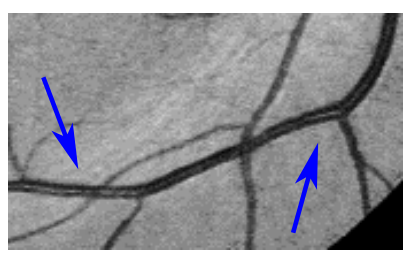

(a)

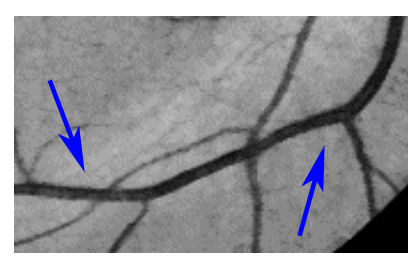

(b)

Figure 3.11: Small opening: (a) Enhanced green component and (b) Opening using $B_{1}$ as $S E$.

Then, principal curvature is calculated as the maximum eigenvalue $\left(\lambda_{\max }\right)$ of the Hessian matrix (Martinez-Perez et al., 2007)

$$
H=\left(\begin{array}{cc}
\partial_{x x} f & \partial_{x y} f \\
\partial_{y x} f & \partial_{y y} f
\end{array}\right)
$$

where $\partial_{i j} f$ represents the second directional derivatives of an image $f(x, y)$. The Hessian matrix is calculated at different scales $(s=$ $\{0,2,8,14\})$ by convolving the original image $f(x, y)$ with a Gaussian kernel $G$ of variance $s^{2}$,

$$
f_{s}(x, y ; s)=f(x, y) \otimes G(x, y ; s)=f(x, y) \otimes \frac{1}{2 \pi s^{2}} e^{-\frac{x^{2}+y^{2}}{2 s^{2}}}
$$

Finally, the principal curvature $f_{\kappa}$ is obtained by normalizing each $\lambda_{\max }$ 
by $2 s$ and computing the local maxima over scales:

$$
f_{\kappa}=\max _{s}\left(\frac{\lambda_{\max }(s)}{2 s}\right) .
$$

If the principal curvature is directly calculated on the enhanced image, all structures with high curvature are highlighted, not only the vessels. The optic disk border has also high curvature but it should not be detected. This is a typical problem that occurs in most edge detection methods for vessel segmentation. To solve this problem, a dual top-hat, with a circular SE larger than the biggest vessel $\left(B_{2}\right)$, is applied with the goal of extracting all of them and eliminating structures with high curvature that are not vessels, as the optic disk. Figure 3.12 illustrates the difference between calculating the principal curvature directly on the enhanced image and on the result of the dual top-hat.

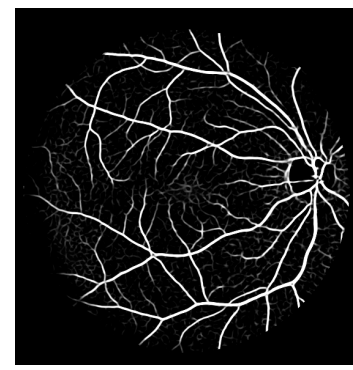

(a)

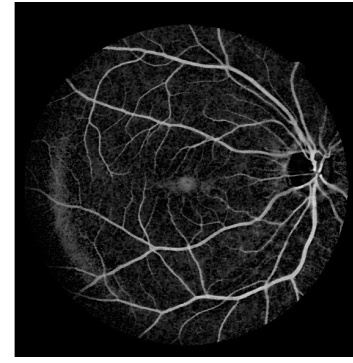

(b)

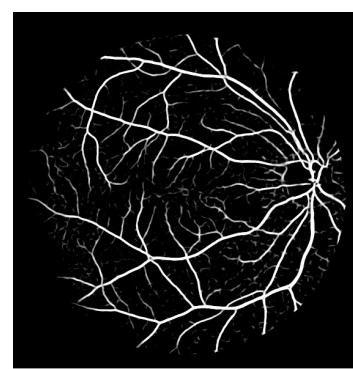

(c)

Figure 3.12: Principal curvature computation: (a) Principal curvature of the enhanced image, (b) Dual top-hat on the enhanced image and (b) Principal curvature of the dual top-hat.

After that, a reconstruction by dilation is applied to reconstruct the principal curvature from a supremum of openings $\left(\gamma_{B}^{\text {sup }}(f)=\right.$ $\left.\bigvee_{i}\left\{\gamma_{B_{i}}(f)\right\}\right)$. In particular, the openings were calculated using a line of size 31 as SE every $15^{\circ}\left(\mathbf{B}_{\mathbf{3}}=\left\{B_{3_{1}}, B_{3_{2}}, \ldots, B_{3_{N}}\right\}\right.$, being $N$ the total number of openings). This operation removes any structure smaller 
than this SE in any orientation obtaining a cleaner background. See Figure 3.13(a) and 3.13(b).

Finally, to binarize the resulting image a k-means clustering (MacQueen, 1967) is used with a $k$ value equals to 3 , giving rise to $\mathbf{f}_{\mathbf{k m}}=\left(f_{1}, f_{2}, f_{3}\right)$. Afterwards, a modification of the k-means output is carried out; two of the three obtained clusters are defined as vessel ( $f_{\text {out }}=f_{1} \cup f_{2}$ ), considering that $f_{3}$ corresponds to the background. Three classes are required because thick and thin vessels can be very different. Figure 3.13(c) depicts the resulting image.

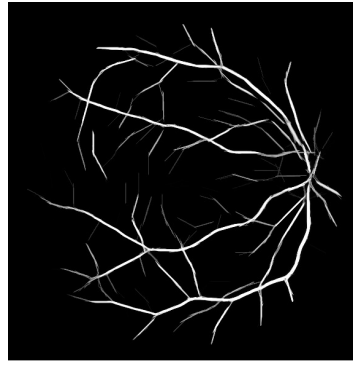

(a)

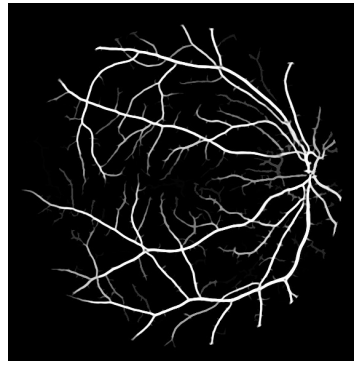

(b)

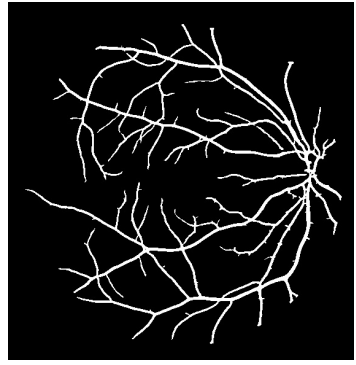

(c)

Figure 3.13: Final vessel segmentation steps: (a) Supremum of openings, (b) reconstruction by dilation and (c) K-means clustering (segmented image).

Algorithm 3.1 summarizes the main steps of the segmentation method.

The method presented in this Subsection as the same way as the method of Zana and Klein et al. (Zana and Klein, 2001) is based on mathematical morphology and curvature evaluation for the detection of the vascular retinal tree. However, the operations used for this purpose are different. Zana and Klein performed a geodesic reconstruction of a supremum of openings to remove noise from the original image while preserving most of the capillaries. Then, a sum of top hats is calculated to improve the contrast of the linear parts. Afterwards, the curvature is computed by means of the Laplacian operator and the final result 


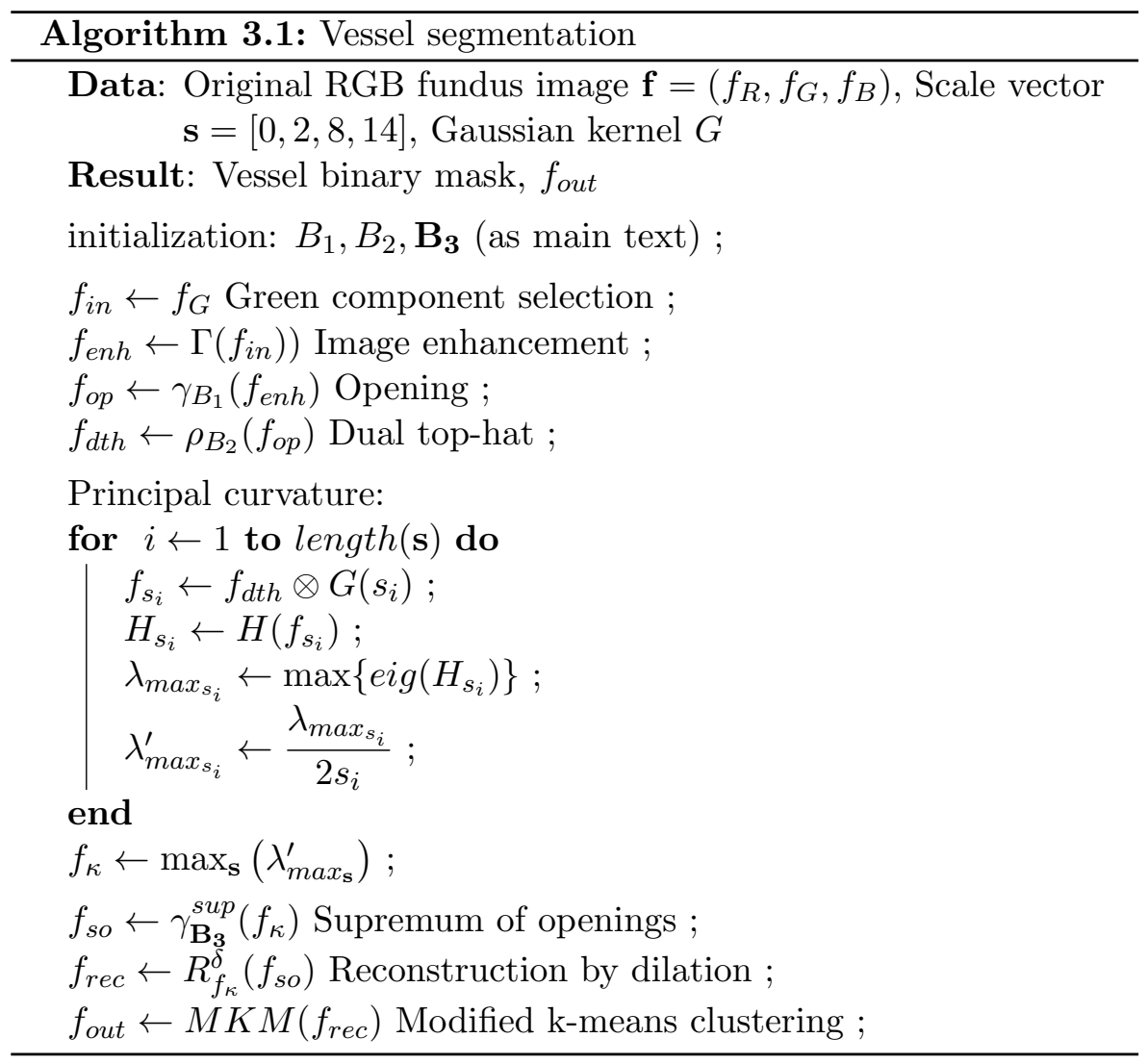

is obtained with an alternating filter. The proposed method calculates the curvature of a dual top-hat and then a reconstruction by dilation is applied to remove noise. The final result is achieved through a k-means clustering.

\subsubsection{Centerline extraction}

In general, the detection of retinal vascular network is necessary before analyzing vessel features. The most common approach in the literature is a first stage of vessel segmentation, then the skeletonization 
of the detected vessels and finally the analysis of different features on the vascular skeleton as vessel caliber or bifurcation angles. The major drawback of this approach is the dependence of the different stages with the previous ones in addition to computational cost. Based on these facts, this subsection is focused on obtaining the retinal skeleton in a direct way avoiding the segmentation stage. Its goal is to reduce the number of necessary steps in the fundus image processing. As a consequence, this would also reduce the dependency of previous stages. Specifically, the method proposed for this purpose is mainly based on mathematical morphology along with curvature evaluation. Two main steps are involved: in the first step, the principal curvature is calculated on the retinal image. In the second step, the stochastic watershed transformation, presented in Subsection 3.2.2, is applied to extract the vascular skeleton. The main stages are included in the flowchart shown in Figure 3.14.

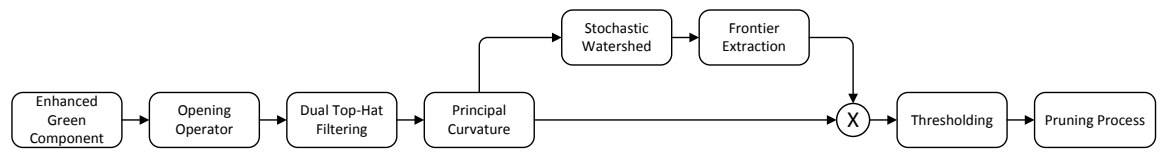

Figure 3.14: Flowchart for skeleton extraction.

The first steps of the method are the same that those included in the method for vessel segmentation presented in Subsection 3.3.1. Although fundus images are RGB format (Figure 3.15(a)), only the green component is used (Figure 3.15(b)). Moreover, this gray image is enhanced by means of the Equation 3.11. Then, a small opening, using a disk of radius 1 as $\mathrm{SE}\left(B_{1}\right)$, is performed on the enhanced green component image to fill in any gaps in vessels that could provoke subsequent errors, for example due to brighter zone within arteries. Next, a dual top-hat, with a SE larger than the widest vessel $\left(B_{2}\right)$, is applied with the goal of extracting all of them and eliminating structures with high gradient that are not vessels, as occurs with the optic disk border (Figure $3.15(\mathrm{c})$ ). Afterwards, with the aim of highlighting the 
vessels on the background, principal curvature, $f_{\kappa}$, is calculated as the maximum eigenvalue of the Hessian matrix (Martinez-Perez et al., 2007) resulting the image shown in Figure 3.15(d).

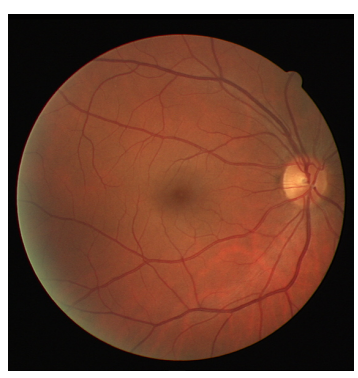

(a)

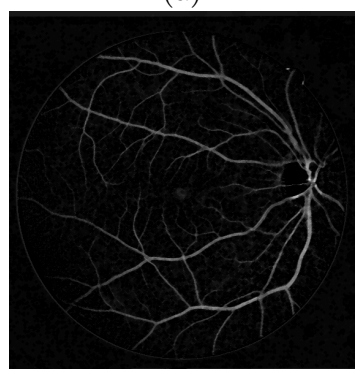

(c)

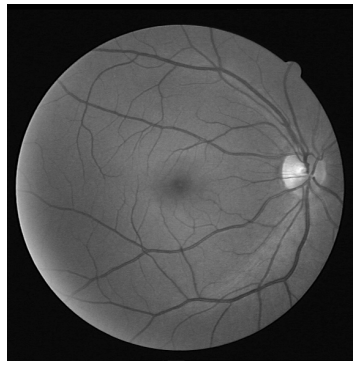

(b)

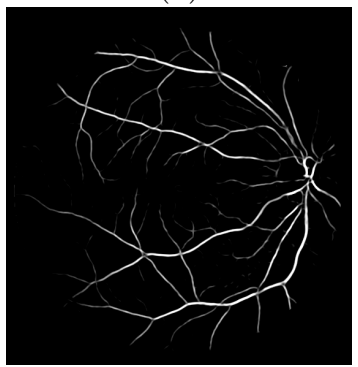

(d)

Figure 3.15: First steps of the skeleton extraction process: (a) Original fundus image, (b) Green component, (c) Dual top-hat filtering and (d) Principal curvature.

However, the following steps are different from those of the previous segmentation method and the stochastic watershed is applied to the curvature image. This transformation uses random markers to build a probability density function ( $p d f$ ) of contours (Figure 3.16(a)), which is then segmented by a last marker-controlled watershed. The vascular skeleton is part of the frontiers of the resultant regions as can be observed in Figure 3.16(b).

In that case, both for $p d f$ obtaining and for last marker-controlled 


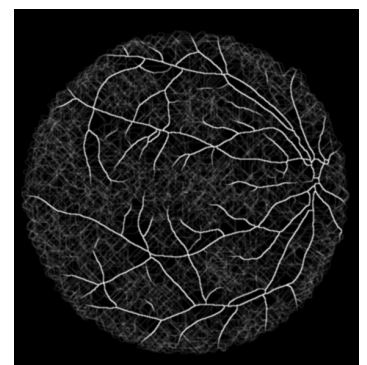

(a)

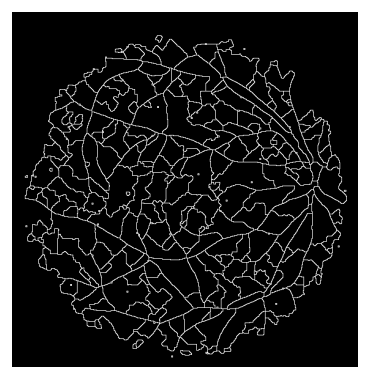

(b)

Figure 3.16: Use of the stochastic watershed for vascular skeleton extraction: (a) Probability density function ( $p d f$ ) of contours obtained with 10 simulations and 300 random markers and (b) Watershed frontiers.

watershed, random markers are combined with some controlled markers. This is due to the morphology of the vascular network which contains multitude of vessel crossings. In addition to the random markers, it is forced that there is one marker at least in the area delimited by the crossing of two vessels (controlled markers). This methodology avoids that the vessels close to some crossing are not detected by the watershed transformation. The crossing areas are determined by means of the residue of the close-hole operator on $f_{\kappa}$ and then one or more markers are chosen randomly within these areas giving place to the controlled markers. This problem is illustrated in Figure 3.17, where only a region of interest is shown for better visualization.

In order to discriminate which watershed frontiers are significant and which ones are not and should be filtered out, the frontiers are multiplied by $f_{\kappa}$ and then are thresholded (Figure 3.18(a)) using a fixed threshold, experimentally $t=0.05$. Once the skeleton is obtained, a pruning process is applied to remove possible spurs giving rise to the final result of the method (Figure 3.18(b) and 3.18(c)).

The implemented pruning process is characterized by removing spur branches but without altering the main branches. Only the branches 


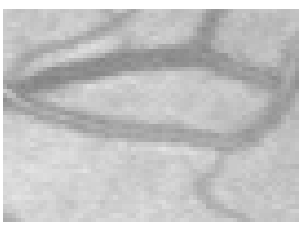

(a)

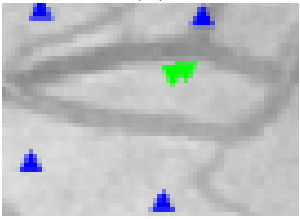

(d)

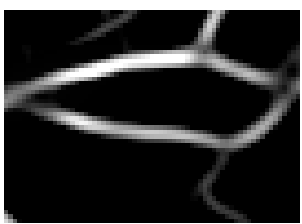

(b)

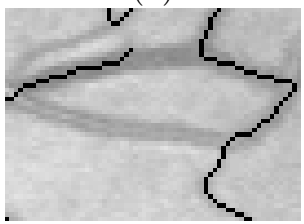

(e)

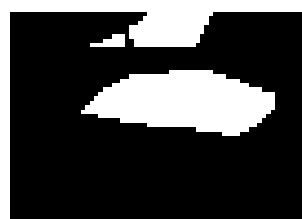

(c)

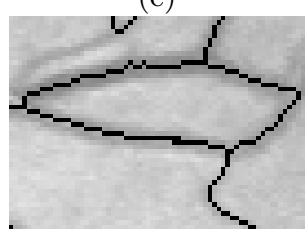

(f)

Figure 3.17: Stochastic watershed on the crossing of two vessels: (a) Enhanced green component, (b) Principal curvature $\left(f_{\kappa}\right),(c)$ Residue of closehole operator, (d) Random (blue $\mathbf{\Delta}$ ) and two controlled (green $\mathbf{\nabla})$ markers, (e) Result of the stochastic watershed using only the random markers shown in blue and $(f)$ Result of the stochastic watershed combining random and controlled markers (blue and green).

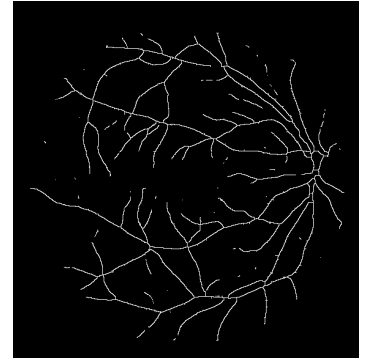

(a)

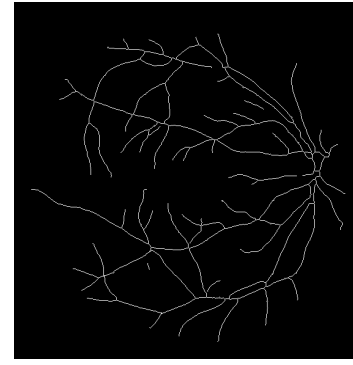

(b)

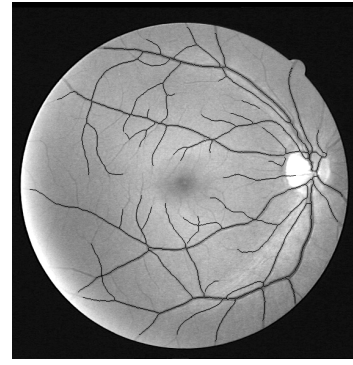

(c)

Figure 3.18: Final steps of the skeleton extraction process: (a) Product between the principal curvature and the watershed frontiers after thresholding, (b) Pruning and (c) Final result.

whose size is less than a threshold $\left(n_{\max }=15\right)$ are removed while the other are kept intact (Walter, 2003). The pruning method is based on 
defining a function $\Upsilon(S)$ which assigns to each point from the skeleton $S$ the number $n$ of thinning sequences needed to remove it from $S$. The value of the function $\Upsilon(S)$ is $n_{\max }+1$ for the points $\mathbf{x} \in S$ which are not removed after $n_{\max }$ thinning processes. Then, making use of the function $\Upsilon(S)$, it is possible differentiate between the secondary and the main skeleton branches. A branch is considered as secondary if $\Upsilon\left(S\left(\mathbf{x}_{1}\right)-\Upsilon\left(S\left(\mathbf{x}_{2}\right)\right)>1\right.$, being $\mathbf{x}_{1}$ and $\mathbf{x}_{2}$ two adjacent points of the skeleton branch. Afterwards, the secondary branches are disconnected from the main branches and a reconstruction by dilation is applied using this image as reference and being the marker image that defined by Equation 3.15. This operation manages to reconstruct the original skeleton but without spur branches. Figure 3.19 shows an example of the main pruning steps.

$$
m r k= \begin{cases}1 & \text { if } \Upsilon(S(\mathbf{x})))=n_{\max }+1 \\ 0 & \text { otherwise }\end{cases}
$$

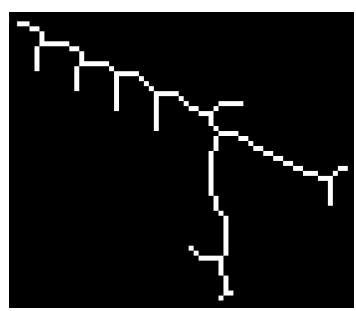

(a)

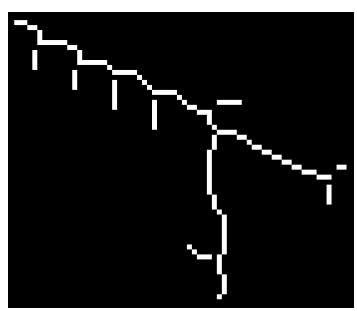

(b)

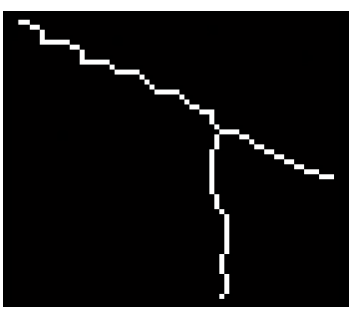

(c)

Figure 3.19: Pruning process: (a) Original skeleton, (b) Skeleton with the secondary branches disconnected and (c) Pruned skeleton.

Algorithm 3.2 summarizes the steps of the vessel centerline extraction method. 


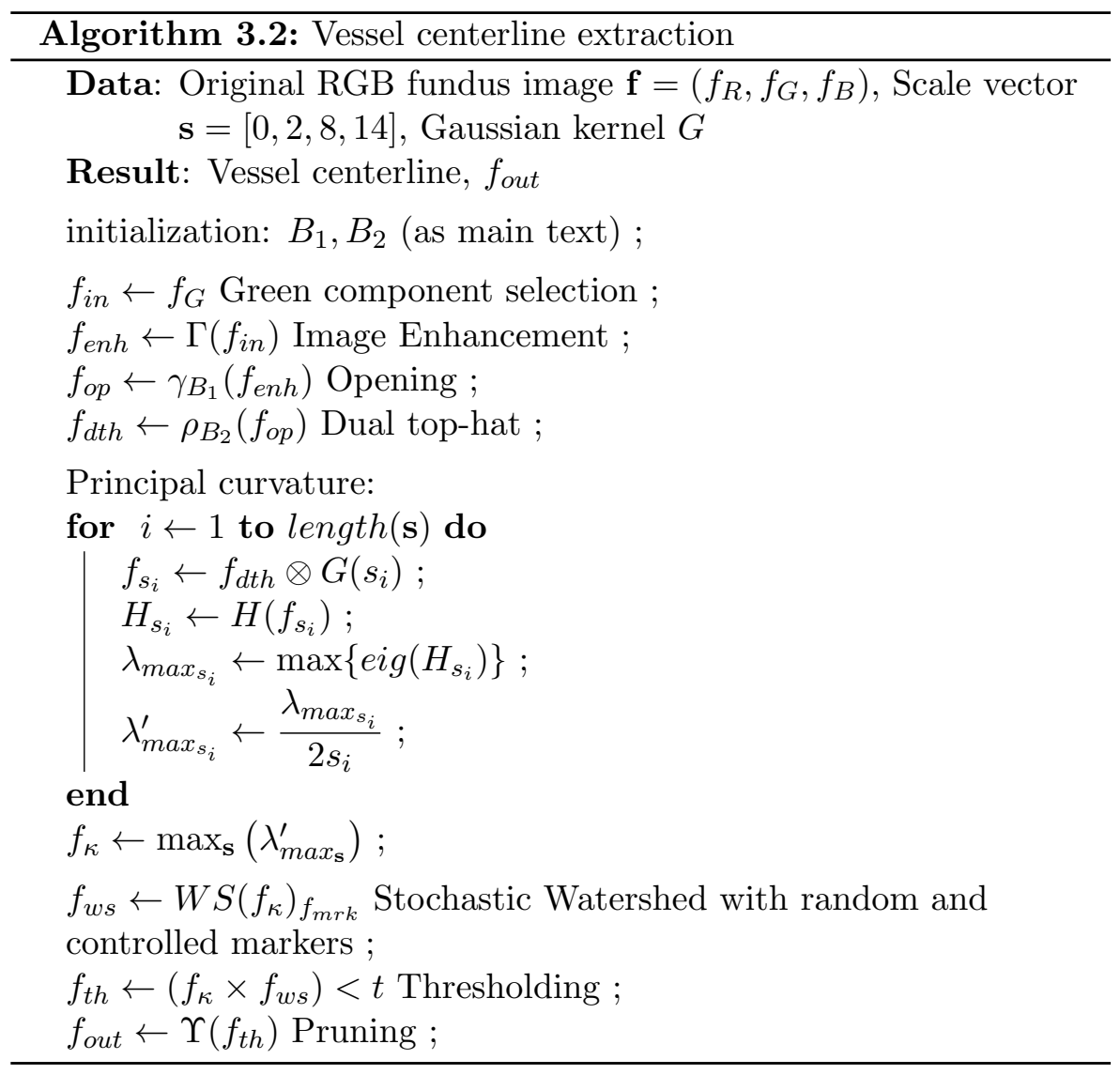

\subsection{Significant points}

This section presents how to detect one of the main features that define and characterize the retinal vascular network: its significant points. The detection of significant points in the retinal vascular tree will increase the information about the vascular structure allowing its use for medical diagnosis (Calvo et al., 2011).

In general, the significant points of the vascular network are detected on vessel centerline. As mentioned in the previous section, the centerline 
can be obtained after a skeletonization process of the vessels previously segmented or through some method by which the skeleton is directly obtained as that presented in Subsection 3.3.2.

In the vascular skeleton there are three types of significant points: terminal, bifurcation and crossing points. All of them must be detected due to their interest to characterize the relations between the different branches of the skeleton, i.e., relations between the parent and daughter branches.

Hit-or-miss transformation (HMT) is a binary morphological operation which is used for detecting specific patterns in an image (Gonzalez et al., 2004). Therefore, it can be applied to detect the significant points on a binary skeleton image. The structuring element (SE) employed in this operation is called composite structuring element since it contains two SE. The first, denoted by $B_{F G}$, defines the set of pixels that should match the foreground (positive pixel values) while the second, denoted by $B_{B G}$, defines the set of pixels that should match the background (zero pixel values). By definition, $B_{F G}$ and $B_{B G}$ share the same origin and are disjoint sets, i.e., $B_{F G} \cap B_{B G}=0$. Depending on whether the origin belongs to $B_{F G}$ or $B_{B G}$ the HMT extracts foreground or background pixels (Soille, 2003). Figure 3.20 depicts a composite structuring element $\mathbf{B}=\left(B_{F G}, B_{B G}\right)$ where $B_{F G}$ is denoted by 1 's, $B_{B G}$ by 0 's and the rest of values are ignored. In this example, the HMT would detect the pixels with a neighbor on the left but that up, down and to the right did not have any. The value of the left diagonals does not matter, it could be 0 or 1 .

\begin{tabular}{|l|l|l|}
\hline & 0 & 0 \\
\hline 1 & 1 & 0 \\
\hline & 0 & 0 \\
\hline \multicolumn{3}{|c}{ B }
\end{tabular}
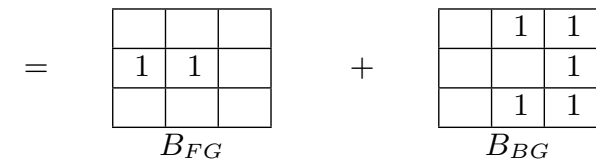

Figure 3.20: Example of a composite structuring element.

The HMT of a set $X$ by a composite structuring element $\mathbf{B}=$ 
$\left(B_{F G}, B_{B G}\right)$ can be written in terms of an intersection of two erosions (Soille, 2003):

$$
\operatorname{HTM}_{B}(X)=\varepsilon_{B_{F G}}(X) \cap \varepsilon_{B_{B G}}\left(X^{c}\right)
$$

where $X^{c}$ is the complement set (i.e., the negative).

\subsubsection{Terminal and bifurcation points}

The hit-or-miss transformation can be directly applied to the vascular skeleton to locate terminal and bifurcation points using the different SE shown in Figure 3.21 and 3.22. It must be remembered that $B_{F G}$ is denoted by 1 's and $B_{B G}$ by 0 's. Note that these SE must be used in all their orientations, one each $90^{\circ}$, so four hit-or-miss iterations are required for each one.

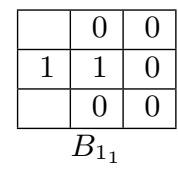

\begin{tabular}{|l|l|l|}
\hline 1 & 0 & 0 \\
\hline 0 & 1 & 0 \\
\hline 0 & 0 & 0 \\
\hline \multicolumn{4}{|c}{$B_{1_{2}}$}
\end{tabular}

Figure 3.21: Structuring elements used for detecting terminal points.
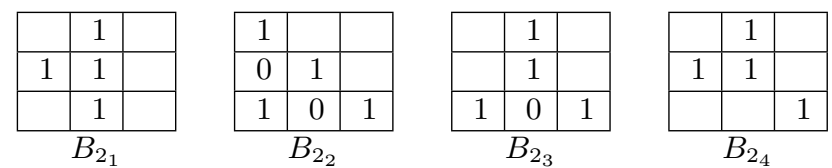

Figure 3.22: Structuring elements used for detecting bifurcation points.

The choice of the SE shape is related to the topology of the point in question. For example, on the one hand, any terminal point will have only one neighbor and it will be round by background pixels and, on the other hand, a bifurcation point will have three neighbors located in 
particular positions. This can be appreciated clearly if the SE defined in Figure 3.22 are observed.

Retinal skeleton is an one-pixel-thick structure fully 8-connected. However, when the significant points belonging to the skeleton are being looked for, it is wanted to avoid the multiple paths that are inherent in this type of connectivity. Therefore, previously to point detection, it is necessary to convert the skeleton from 8 -connectivity to $m$-connectivity so that the multiple paths are removed.

A pixel $p$ has four horizontal and vertical neighbors $N_{4}(p)$ and four diagonal neighbors $N_{D}(p)$. All these neighbors are called the 8neighbors of $p$, denoted by $N_{8}(p)$. Two binary pixels $p$ and $q$ are 8connected if $q$ is in the set $N_{8}(p)$ but they are m-connected if

1. $q$ is in $N_{4}(p)$, or

2. $q$ is in $N_{D}(p)$ and $N_{4}(p) \cap N_{4}(q)=0$.

The difference between 8-connectivity and $m$-connectivity can be appreciated in Figure 3.23. This conversion is necessary because the central pixel makes that there is not only a possible path in the skeleton therefore the detection of significant points and any tracking process performed later could be erroneous.

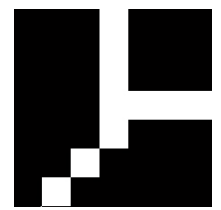

(a)

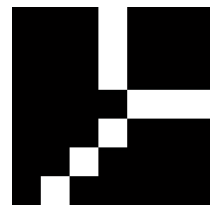

(b)

Figure 3.23: 8-connectivity to m-connectivity conversion (a) 8-connected skeleton and (b) m-connected skeleton.

In the case of the 8-connectivity, the multiple paths are manifested in 
four basic patterns which are shown in Figure 3.24. It can be observed that the case shown in Figure 3.23 corresponds to the patterns $B_{3_{2}}$ and $B_{3_{3}}$.
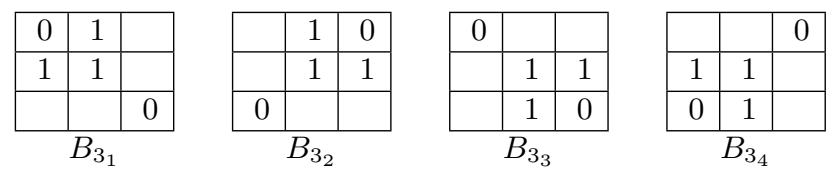

Figure 3.24: Structuring elements used to convert an 8-connected skeleton to m-connectivity.

The HMT allows to detect all these patterns. Then, the central pixels must be changed to 0 for eliminating the multiple paths. The conversion from 8 to m-connectivity can be performed through a basic sequence of morphological steps:

$$
\begin{aligned}
& \Theta_{1}\left(X, B_{3_{1}}\right)=X-H M T_{B_{3_{1}}}(X)=X \cap\left[H M T_{B_{3_{1}}}(X)\right]^{c} \\
& \Theta_{2}\left(\Theta_{1}, B_{3_{2}}\right)=\Theta_{1}-H M T_{B_{3_{2}}}\left(Y_{1}\right)=Y_{1} \cap\left[H M T_{B_{3_{2}}}\left(Y_{1}\right)\right]^{c} \\
& \Theta_{3}\left(\Theta_{2}, B_{3_{3}}\right)=\Theta_{2}-H M T_{B_{3_{3}}}\left(Y_{2}\right)=Y_{2} \cap\left[H M T_{B_{3_{3}}}\left(Y_{2}\right)\right]^{c} \\
& \Theta_{4}\left(\Theta_{3}, B_{3_{4}}\right)=\Theta_{3}-H M T_{B_{3_{4}}}\left(Y_{3}\right)=Y_{3} \cap\left[H M T_{B_{3_{4}}}\left(Y_{3}\right)\right]^{c} \\
& \Theta\left(X, \mathbf{B}_{3}\right)=\Theta_{4},
\end{aligned}
$$

where $X$ is the input image that contains the 8-connected skeleton and $\Theta$ the output image with the corresponding skeleton with $m$ connectivity.

\subsubsection{Crossing points}

Due to the fact that the intersections between different branches of the vascular tree are formed, usually, by a set of pixels, most crossing points cannot be detected by pattern recognition on skeleton, i.e., 
through the hit-or-miss transformation. It can only be applied in simple crossing point detection using the SE shown in Figure 3.25.

$$
\begin{array}{|l|l|l|}
\hline 0 & 1 & 0 \\
\hline 1 & 1 & 1 \\
\hline 0 & 1 & 0 \\
\hline \multicolumn{4}{|c}{B_{4_{1}}}
\end{array}
$$

\begin{tabular}{|l|l|l|}
\hline 1 & 0 & 1 \\
\hline 0 & 1 & 0 \\
\hline 1 & 0 & 1 \\
\hline \multicolumn{4}{|c|}{$B_{4_{2}}$}
\end{tabular}

Figure 3.25: Structuring elements used for detecting simple crossing points.

However, practically most, if not all crossing points are not simple, or in other words, the branches do not intersect in only one pixel but several points can belong to the same intersection. This provokes that the extremes of the intersection are considered as bifurcation because both of them have three neighbors and accomplishes some of the characteristic pattern of the bifurcation points. Figure 3.26 represents the different types of crossing points. The light gray branch intersects with other two branches drawn in dark gray giving rise to a simple $(\mathrm{X})$ and a complex $(\mathrm{O})$ crossing point marked in black.

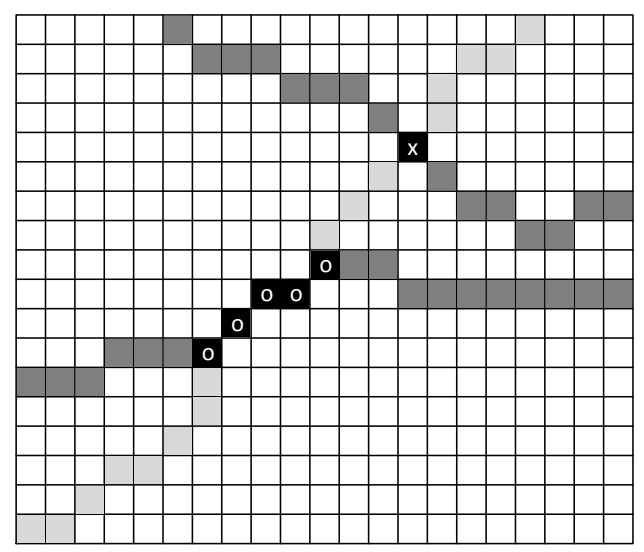

Figure 3.26: Different types of crossing points: simple $(X)$ and complex $(O)$.

Most works of the state of the art consider that the vessel crossing 
points are two bifurcation points very close to each other. So, a fixedsize circular window is centered on the candidate bifurcations and if there exist four intersections between the window and the skeleton, the point is marked as crossing. The problem of this approach is that the crossing point detection depends on a large degree on the window size. If the size is too small, the crossings are not detected and if the size is too big other vessels not belonging to this crossing can intersect with the window. Moreover, it must be taken into account that the size of the intersections vary from one case to another.

Retinal vessels have their origin in the optic disk head. From this center, the vessels bifurcate and constitute the retinal vascular tree. It is common that arteries and veins intersect in some occasion and generate the crossing points under consideration (Figure 3.27(a)). This means that when they intersect, as the vessels have a common origin, generate a sort of close loop which will be useful to differentiate if one point is a crossing point or not (Figure 3.27(b)). Based on this idea, a new algorithm is proposed to analyze all points detected initially as bifurcation in order to discriminate those that are really crossing points.

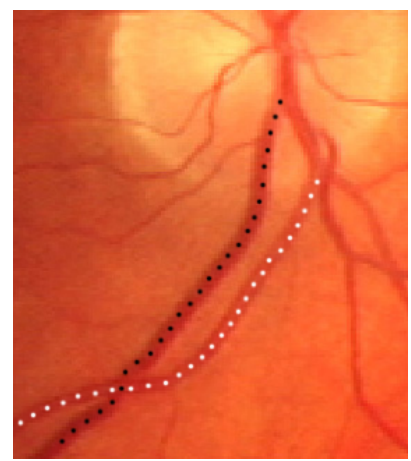

(a)

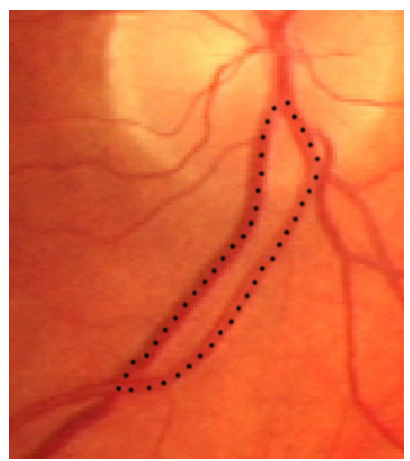

(b)

Figure 3.27: Vessel intersection: (a) Intersection between two vessels and (b) Close loop formed by an intersection.

First, a bifurcation point is considered as candidate to crossing 
point if when a circular window is centered on this point, there are four or more intersections between the skeleton and this window $(W)$. The window radius will be three times the median of the diameter of the vessels in all points detected initially as bifurcation. This size was established empirically. Then, if the candidate point is part of a close loop generated by the skeleton branches, the closest candidate to crossing point is looked for, and if it is directly connected with the previous point and is not part of the same loop, both of them are established as crossing points. Figure 3.28 represents this process. Terminal and bifurcation points detected by the HMT are marked in red and green, respectively (Figure 3.28(a)). In Figure 3.28(b) the candidates to crossing points are highlighted in yellow. The close loops that contain some candidate point are drawn in Figure 3.28(c). Figure $3.28(\mathrm{~d})$ shows the final result with the crossing points detected in white.

With this type of analysis, the more common intersection extremes are completely identified. In addition, it should be taken into account that the pixels between these points are also part of the same intersection.

Algorithm 3.3 summarizes the complete process of the detection of the significant points on the retinal vascular skeleton. $\mathbf{B}_{\mathbf{1}}, \mathbf{B}_{\mathbf{2}}, \mathbf{B}_{\mathbf{3}}, \mathbf{B}_{\mathbf{4}}$ are the composite structuring elements defined in Figures 3.21, 3.22, 3.24 and 3.25 .

\subsection{Optic disk segmentation}

Optic disk (OD) segmentation is a key process in many algorithms designed for the automatic extraction of anatomical ocular structures, the detection of retinal lesions, and the identification of other fundus features. The following examples can be stood out. The OD location helps to avoid false positives in the detection of exudates associated with diabetic retinopathy, since both of them are spots with similar intensity (Walter et al., 2002). The OD margin can be used for establishing standard and concentric areas in which retinal 


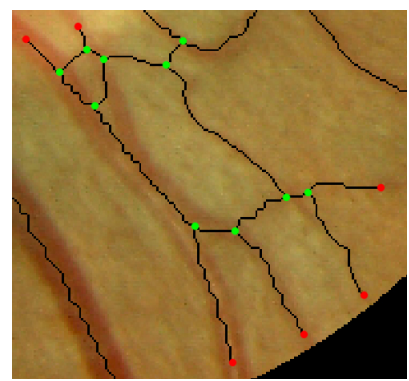

(a)

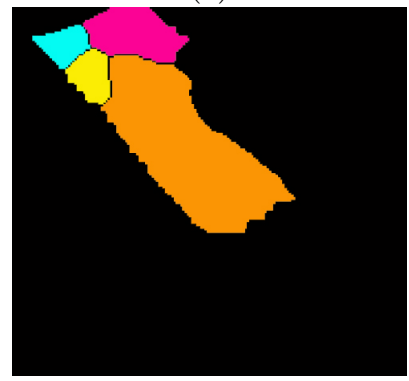

(c)

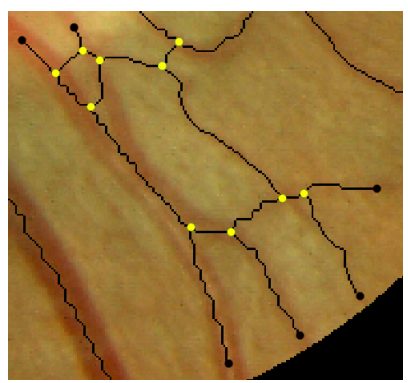

(b)

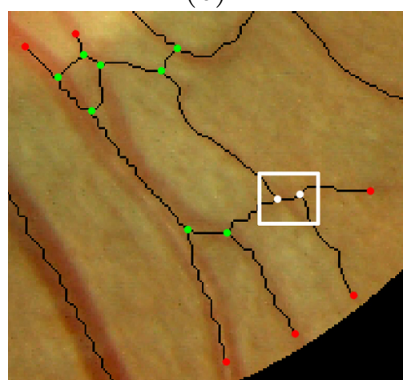

(d)

Figure 3.28: Automatic detection of common intersections: (a) Significant points detected by means of HMT (terminal points in red and bifurcation points in green), (b) Crossing point candidates, (c) Close loops formed by branches that contain some candidate point and (d) Crossing points automatically detected (white).

vessel diameter measurements are performed by calculating some important diagnostic indexes for hypertensive retinopathy, such as central retinal artery equivalent (CRAE) and central vein equivalent (CRVE) (Hubbard et al., 1999; Knudtson et al., 2003). The relation between the area of the optic disk and the optic cup is used for glaucoma diagnosis (Liu et al., 2009; Hatanaka et al., 2010). The relatively constant distance between the OD and the fovea is useful for estimating the location of the macula (Niemeijer et al., 2009). The center, or even the border, of the OD also serves as initial point for vessel tracking algorithms due to the fact that all retinal vessels are originated from 


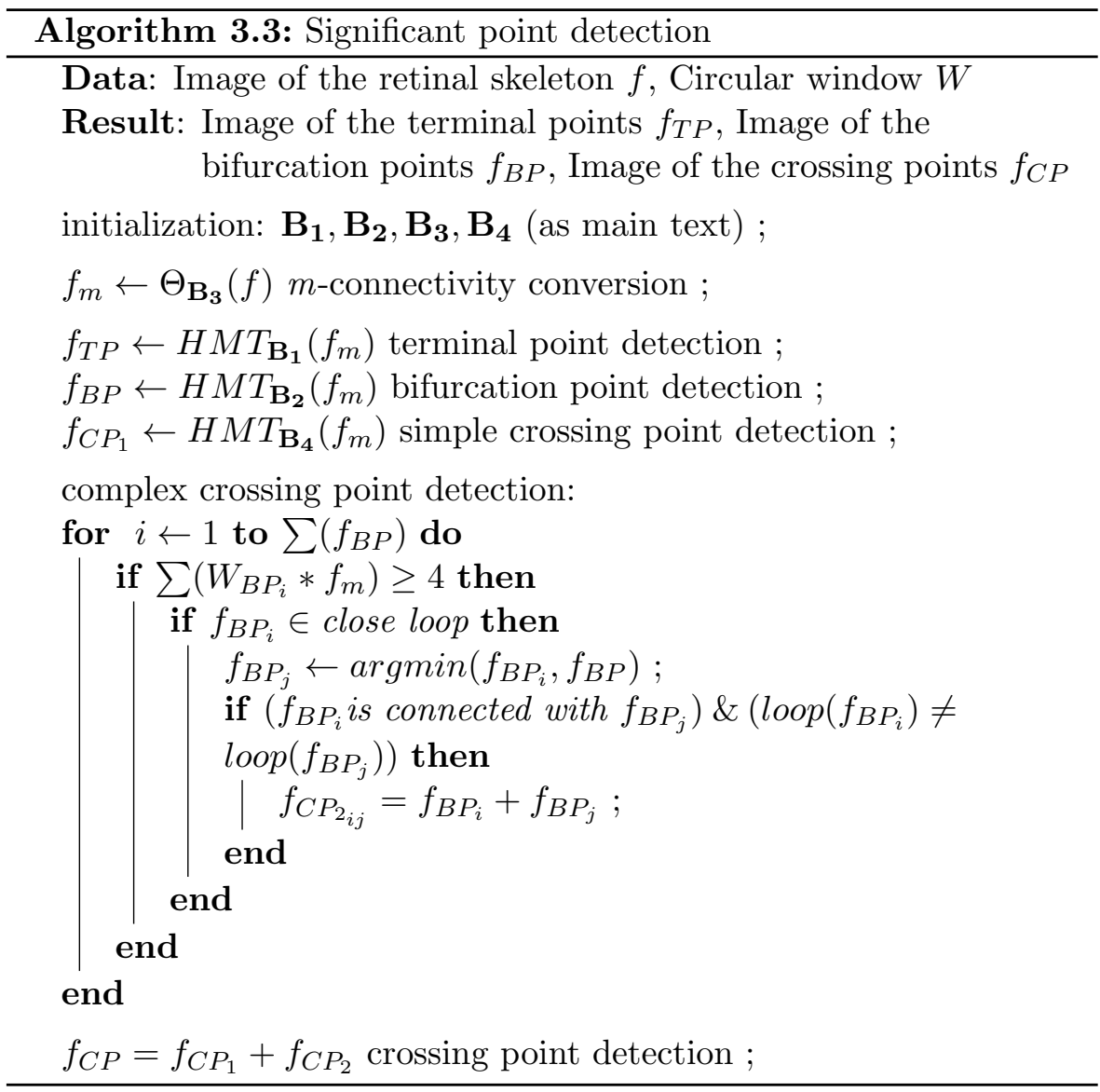

there (Gagnon et al., 2001).

The automatic method proposed for optic disk segmentation is focused on the stochastic watershed using different operations based on mathematical morphology on a fundus image to obtain the ODcontour. Previously, a pre-processing of the original RGB image is required. The first step of the pre-processing consists of applying PCA to transform the input image to gray scale. This technique combines the most significant information of the three components RGB in a 
single image so that it is a more appropriate input to the segmentation method. After segmentation, a post-processing is also performed to fit the final region contour by a circumference. In Figure 3.39, the block diagram of the complete segmentation process is depicted.

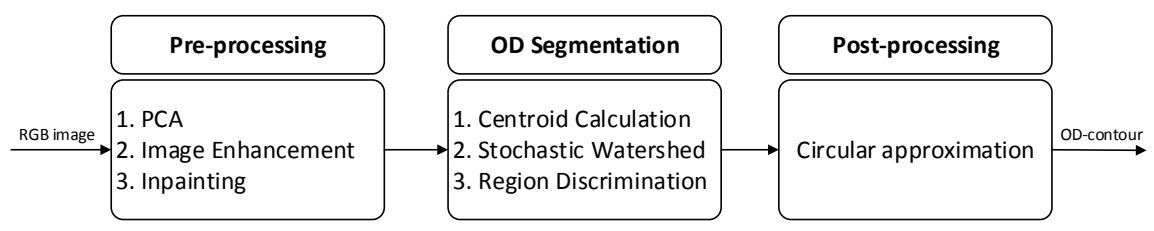

Figure 3.29: Optic disk segmentation diagram.

\subsubsection{Pre-processing}

\section{PCA}

Generally, an initial gray-scale image is necessary to carry out most of the segmentation algorithms of the literature. However, in the case of the OD segmentation, each author considers appropriate a different intensity image, such as a band of the original RGB image (Niemeijer et al., 2009; Walter et al., 2002) or a component of other color spaces (Lowell et al., 2004; Osareh et al., 2002). In this work, the use of a new gray-scale image is proposed. Specifically, it is calculated by means of principal component analysis (PCA) because this type of analysis maximizes the separation of the different objects that compose an image so that the structures of the retina are better appreciated. In addition, it is much less sensitive to the existing variability in a fundus image regarding color, intensity, etc.

The central idea of PCA is to reduce the dimensionality of a data set consisting of a number of interrelated variables, while retaining as much as possible of the variation present in the data set. This is achieved by transforming to a new set of variables, the principal components (PCs), 
which are uncorrelated, and ordered so that the first few retain most of the variations present in all of the original variables (Jolliffe, 2002).

Suppose that $\mathbf{v}$ is a vector of $\mathrm{p}$ random variables with a mean equal to 0 , the $k$ th PC, $z_{k}$, will be a linear function $\boldsymbol{\alpha}_{k}{ }^{\prime} \mathbf{v}$ of the elements of $\mathbf{v}$ where $\boldsymbol{\alpha}_{k}$ is a vector of $p$ constants $\alpha_{k_{1}}, \alpha_{k_{2}}, \ldots, \alpha_{k_{p}}$ and 'denotes transpose

$$
z_{k}=\boldsymbol{\alpha}_{k}{ }^{\prime} \mathbf{v}=\alpha_{k_{1}} v_{1}+\alpha_{k_{2}} v_{2}+\ldots+\alpha_{k_{p}} v_{p}=\sum_{j=0}^{p} \alpha_{k_{j}} v_{j}
$$

$\boldsymbol{\alpha}_{1}{ }^{\prime} \mathbf{v}$ will have maximum variance, $\boldsymbol{\alpha}_{2}{ }^{\prime} \mathbf{v}$ will be uncorrelated with $\boldsymbol{\alpha}_{1}{ }^{\prime} \mathbf{v}$ and will have maximum variance, and so on, so that at the $k$ th stage $\boldsymbol{\alpha}_{k}{ }^{\prime} \mathbf{v}$ will have maximum variance subject to being uncorrelated with $\boldsymbol{\alpha}_{1}^{\prime} \mathbf{v}, \boldsymbol{\alpha}_{2}^{\prime} \mathbf{v}, \ldots, \boldsymbol{\alpha}_{k-1}^{\prime} \mathbf{v}$.

For a three-channel image transforming to a principal component space creates three new channels in which the first (the most significant) contains the most structural contrast and information. The rank for each axis in the principal set represents the significance of that axis as defined by the variance in the data along that axis. Thus, the first principal axis is the one with the greatest amount of scatter in the data and consequently the greatest amount of contrast and information, while the last principal axis represents the least amount of information such as noise and image artefacts (Russ, 2007). In this case, the PCs are given by

$$
z_{k}=\boldsymbol{\alpha}_{k}{ }^{\prime} \mathbf{f}=\alpha_{k_{R}}\left(f_{R}-\bar{f}_{R}\right)+\alpha_{k_{G}}\left(f_{G}-\bar{f}_{G}\right)+\alpha_{k_{B}}\left(f_{B}-\bar{f}_{B}\right),
$$

where $\mathbf{f}(\mathbf{x})=\left(f_{R}(\mathbf{x}), f_{G}(\mathbf{x}), f_{B}(\mathbf{x})\right)$ represents an RGB image, $\bar{f}_{i}$ is the mean of each RGB component and $k \in\{1,2,3\}$. 
Specifically, principal-component axes $\left(\boldsymbol{\alpha}_{k}\right)$ will be the eigenvectors of the covariance matrix $\boldsymbol{\Sigma}$. This is the matrix whose $(i, j)$ th element is the covariance between the $i$ th and $j$ th elements of $\mathbf{f}$ when $i \neq j$, and the variance of the $j$ th element of $\mathbf{f}$ when $i=j$ according to

$$
\boldsymbol{\Sigma}=\left(\begin{array}{ccc}
\sigma_{R R} & \sigma_{R G} & \sigma_{R B} \\
\sigma_{G B} & \sigma_{G G} & \sigma_{G B} \\
\sigma_{R B} & \sigma_{G B} & \sigma_{B B}
\end{array}\right)
$$

Being:

$$
\sigma_{m n}=\frac{1}{N} \sum_{i=1}^{N}\left(f_{m i}-\bar{f}_{m}\right)\left(f_{n i}-\bar{f}_{n}\right)
$$

where $N$ is the number of pixels of the image, $(m, n)$ represent the possible combinations between the RGB components and $\bar{f}_{m}$ and $\bar{f}_{n}$ determine the mean of the corresponding component, respectively.

Therefore, $z_{1}, z_{2}$, and $z_{3}$ are the PCs of the image $\mathbf{f}$ so that $\boldsymbol{\alpha}_{1}$ is the eigenvector corresponding to the largest eigenvalue of $\boldsymbol{\Sigma}, \boldsymbol{\alpha}_{2}$ corresponding to the second largest eigenvalue, and $\boldsymbol{\alpha}_{3}$ corresponding to the smallest one. Figure 3.30 shows the PCs of a RGB fundus image where can be appreciated that the first PC contains the most structural contrast and information.

The first $\mathrm{PC}$ is the most significant so, $z_{1}$ is chosen as the input image of the method. It must be stressed that to ensure that $z_{1}$ contains the most structural contrast and information of the original RGB channels, it should be verified that the largest eigenvalue represents at least a $90 \%$ of the total sum of eigenvalues. In spite of this situation is not often in the consulted databases, if the largest eigenvalue represents less than $90 \%$, the components whose sum of eigenvalues get the $90 \%$ must be processed in parallel. Afterwards, the input of the watershed transformation will be the pixel-wise maximum of the gradient images. 


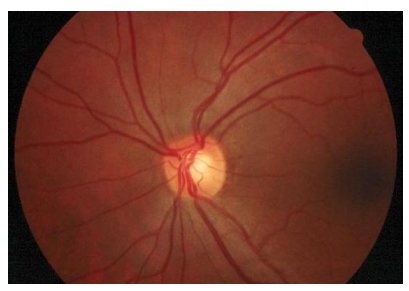

(a)

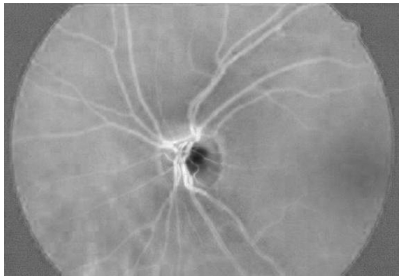

(c)

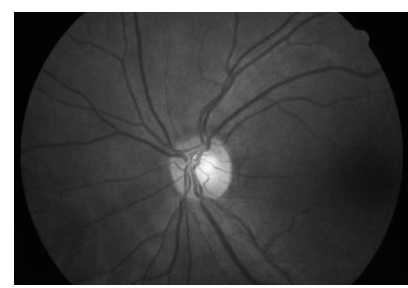

(b)

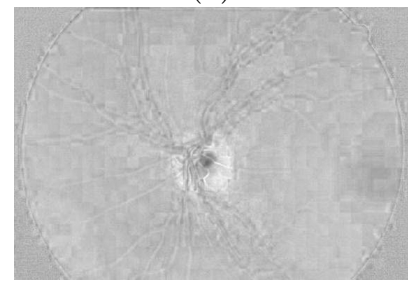

(d)

Figure 3.30: Principal components (PCs): (a) Original fundus image, (b) First $P C z_{1}$, (c) Second $P C z_{2}$ and (d) Third $P C z_{3}$.

The non-uniform illumination of this gray image is corrected by means of the local transformation $(\Gamma)$ of the Equation 3.11. $\Gamma$ is applied on $z_{1}$ resulting in an enhanced image

$$
z_{1}^{\prime}=\Gamma\left(z_{1}\right)
$$

\section{Inpainting}

Retinal vessels are originated from the OD therefore numerous vessels cross its border which makes its discrimination difficult. So, vessel removal of the enhanced image is implemented by an inpainting technique.

Inpainting algorithms are used in diverse applications, from the restoration of damaged photographs to the removal/replacement of selected objects (Guillemot and Le Meur, 2014). These algorithms 
usually try to fill selected parts of an image by propagating external information so that structure continuity is preserved.

Although there exist numerous inpainting techniques (Liang et al., 2001; Levin et al., 2003; Telea, 2004; Mairal et al., 2008), a simple diffusion-based algorithm was implemented in this work. Let a binary image $\Omega(\mathbf{x})$ stand for the region to be inpainted and $\partial \Omega$ for its boundary. For each $\partial \Omega$-pixel, $\mathbf{x}$, the inpainted-pixel value is computed as

$$
P(\mathbf{x})=\frac{\sum_{k=1}^{n} \frac{P_{k}(\mathbf{x})}{l_{k}}}{\sum_{k=1}^{n} \frac{1}{l_{k}}},
$$

where $P_{k}$ denotes the pixel values in a $5 \times 5$ neighborhood of the pixel under consideration, $n$ is the number of neighboring pixels, and $l_{k}$ is the distance between the pixel $\mathbf{x}$ and each neighboring pixel. So that the inpainted image $\Psi(f, \Omega)(\mathbf{x})$ of a gray image $f(\mathbf{x})$ is

$$
\Psi(f, \Omega)(\mathbf{x})= \begin{cases}f(\mathbf{x}) & \text { if } \partial \Omega(\mathbf{x})=0 \\ P(\mathbf{x}) & \text { if } \partial \Omega(\mathbf{x})=255 .\end{cases}
$$

After filling $\partial \Omega$ with the computed values, the $\partial \Omega$-pixels are removing from $\Omega$ and $\partial \Omega$ is recalculated. The process is repeated until the mask is empty and all pixels are filled.

In particular, the region to be inpainted $\Omega$ is a binary mask of the retinal vessels, which must have been segmented previously. The method used for vessel segmentation is that proposed in Subsection 3.3.1. Afterwards, a unitary morphological dilation of the segmented vessels yields the final vessel mask. The purpose of this operation is to make sure that the vessels will be contained in the mask. 
The aim of applying the inpainting is to extract the OD-boundary more precisely and to reduce the existing borders within the OD which increase the risk of under-segmentation. This operation gives place to

$$
z_{1}^{\prime \prime}=\Psi\left(z_{1}^{\prime}, \Omega\right)
$$

being $z_{1}^{\prime}$ the enhanced image (Equation 3.22), $\Omega$ the binary image with the segmented vessels and $z_{1}^{\prime \prime}$ the inpainted image.

In Figure 3.31 the pre-processing of the original image, shown in Figure 3.30(a), can be observed, both the obtaining of the first PC and its enhancement by means of non-linear transformation $\Gamma$ and also the vessel removal. Note that the whole image is processed although only a region of interest is shown for better visualization.

\subsubsection{Processing}

\section{Marker selection}

As mentioned above, the segmentation method makes use of the stochastic watershed. This transformation uses random markers to build a probability density function $(p d f)$ of contours, according to Equation 3.9, which is then segmented by volumic watershed to define the most significant regions. However, in the marker definition not only internal markers (that specify what is the object of interest) are needed, but also an external marker which limits the area to be segmented.

On the one hand, the chosen external marker, $f_{\text {ext }}$, will be a circle of constant diameter centered on the centroid of the optic disk. The size of the circle is related to the image size, so that it is approximated by a $15 \%$ of the size of the fundus image (with this size we made sure that the optic disk is included). The challenge is to be able to find the OD centroid prior to being segmented. For this purpose, we work with the centroid of a gray-level image. 


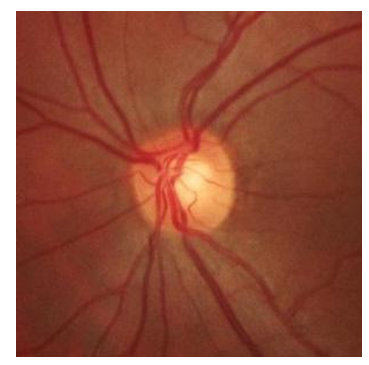

(a)

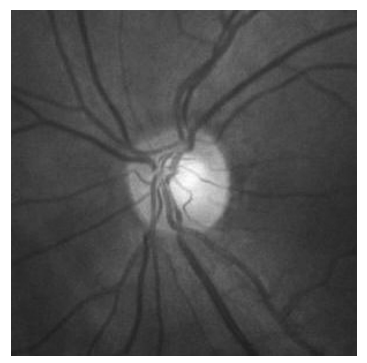

(b)

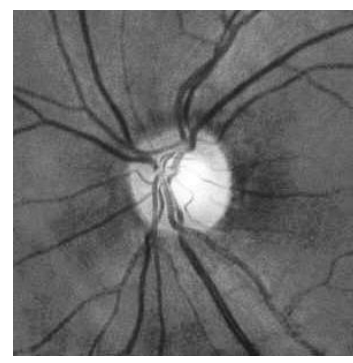

(c)

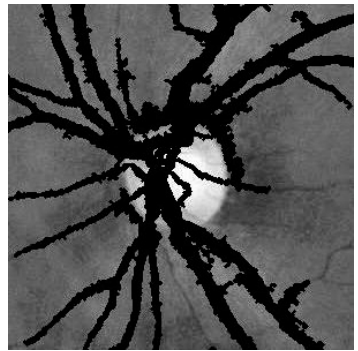

(d)

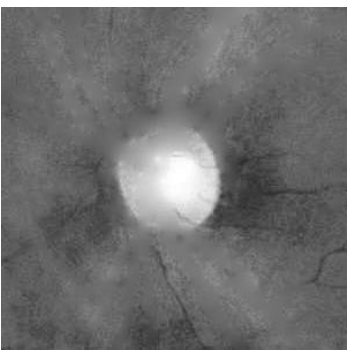

(e)

Figure 3.31: OD pre-processing: (a) Original image, (b) First $P C z_{1}$, (c) Enhanced image $z_{1}^{\prime}$, (d) Vessel mask to be inpainted $\Omega$, and (e) Inpainted image $z_{1}^{\prime \prime}$.

The centroid of a gray-level image can be calculated based on the generalized distance function (GDF) (Vincent, 1998). This algorithm is focused on modifying the classic two-pass sequential distance function (Rosenfeld and Pfaltz, 1968) so that: (1) edge cost is taken into account; (2) raster and anti-raster scans of the image are iterated until stability. Let $N^{+}(p)$ (resp., $N^{-}(p)$ ) be the 8-connected neighborhood of pixel $p$ scanned before $p$ (resp., after $p$ ) in a raster scan, and $C_{f}(p, q)=f(p)+f(q)$ the associated cost to two neighboring pixels $p$ and $q$, the algorithm of GDF to set $X$ in an image $f$ proceeds as follows:

- Initialize result image $d_{f}: d_{f}(p)=0$ if $p \in X$ and $d_{f}(p)=+\infty$ 
otherwise.

- Iterate until stability, for each pixel $p$ :

- Scan image in raster order: $d_{f}(p) \leftarrow \min \left\{d_{f}(p), \min \left\{d_{f}(q)+C_{f}(p, q), q \in N^{+}(p)\right\}\right\}$.

- Scan image in anti-raster order: $d_{f}(p) \leftarrow \min \left\{d_{f}(p), \min \left\{d_{f}(q)+C_{f}(p, q), q \in N^{-}(p)\right\}\right\}$.

More specifically, the gray-level centroid of an image $f$ can be obtained as the maximum of the GDF to the image border set $B$ as follows:

- Compute GDF to set $B$ in image $f, d_{f}(B)$.

- Find the maximal value of $d_{f}(B), u_{\max }$.

- Threshold $d_{f}(B)$ with the $u_{\max }$ calculated to define set $C$ as image centroid.

- If $C$ has more than one pixel, compute the centroid of set $C$ as the maximum edge distance.

This way for calculating the gray-image centroid combines the centrality of the image with respect to edge distance (i.e. purely geometric) but penalizing this distance in relation to the intensities. Thus, note that it cannot be defined as the center of mass of the intensities or as the center of the brighter and larger zone, since the two effects are combined. In Figure 3.32 the result of applying the algorithm on the first principal component of the original image after inpainting technique can be appreciated.

If Figure 3.32(c) is observed, we can see that the image centroid does not match with the OD centroid. To obtain the desired result, the optic disk should be a brighter zone than the background (sufficiently 


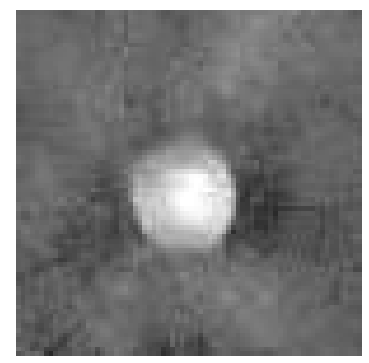

(a)

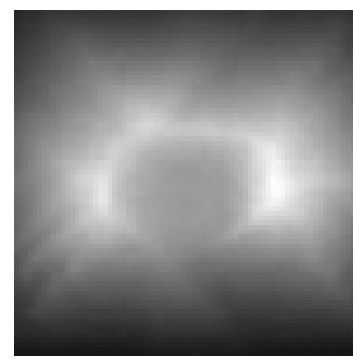

(b)

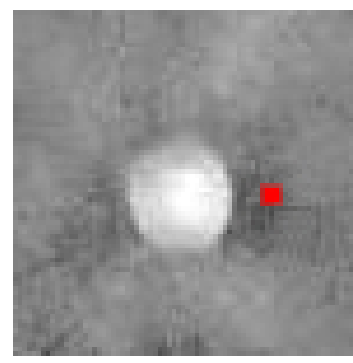

(c)

Figure 3.32: Gray-image centroid on $z_{1}$ : (a) First $P C\left(z_{1}\right)$ after inpainting technique, (b) GDF of (a), and (c) Image centroid.

contrasted) and should have a significant size with respect to the image size. So, to accomplish this condition, the algorithm should be computed on the residue of a close holes of the original gray image as shown Figure 3.33.

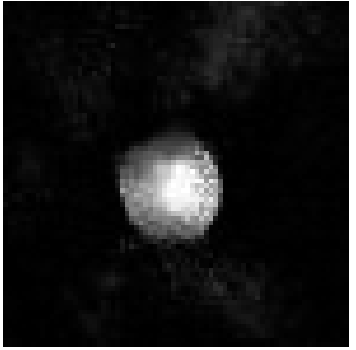

(a)

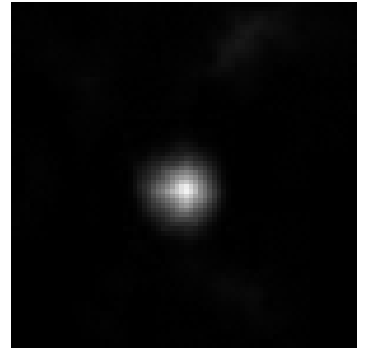

(b)

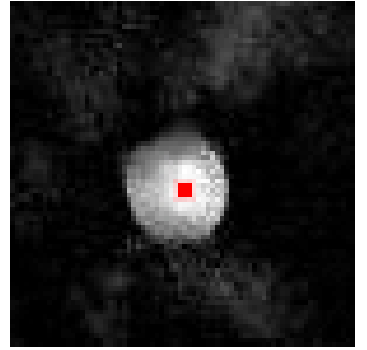

(c)

Figure 3.33: Gray-image centroid on the residue of close operator: (a) Residue of close operator of Figure 3.32(a), (b) GDF of (b), and (c) Image centroid.

On the other hand, the internal markers, $f_{\text {int }}$, will be uniform random markers generated within the area limited by $f_{\text {ext }}$. See Figure 3.34. Hence, the final set of markers $f_{m}$ (external and internal) is the 
logical OR of both of them,

$$
f_{m}=f_{\text {int }} \vee f_{\text {ext }}
$$

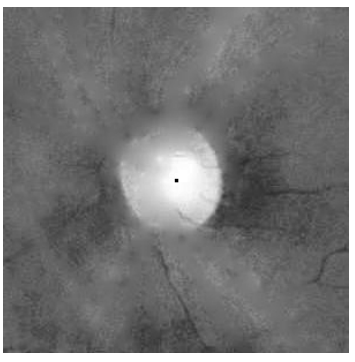

(a)

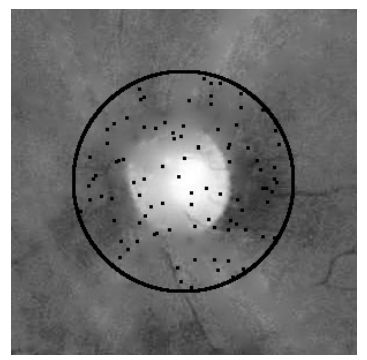

(b)

Figure 3.34: Final markers: (a) Centroid image and (b) Uniform random markers limited by an external circle centered on the image centroid.

\section{Stochastic watershed transformation}

In particular, the $p d f$ is built from 15 marker-controlled-watershed realizations, as showed in Subsection 3.2.2, using as input the gradient of the inpainted image $\varrho\left(z_{1}^{\prime \prime}\right)$ (Figure 3.35(a)). Therefore, in that case, the $i$ th watershed output $W S_{i}=W S\left(\varrho\left(z_{1}^{\prime \prime}\right)\right)_{f_{m_{i}}}$, being $f_{m_{i}}$ the final set of markers used in the corresponding simulation. $f_{\text {int }}$ is generated for each simulation while $f_{\text {ext }}$ is the same in all of them. The number of internal markers used is $N=100$.

Obtaining a $p d f$ of the contours of the watershed regions (Figure 3.35(b)) facilitates the final segmentation, providing robustness and reliability since the arbitrariness in choosing the markers is avoided. Afterwards, the $p d f$ can be combined with the initial gradient in order to reinforce the gradient contours which have a high probability resulting 
a probabilistic gradient (Angulo and Jeulin, 2007),

$$
\rho\left(z_{1}^{\prime \prime}\right)(\mathbf{x})=(1-\lambda) \varrho\left(z_{1}^{\prime \prime}\right)(\mathbf{x})+\lambda p d f(\mathbf{x})
$$

with $\lambda=0.5$ the results are in general satisfactory.

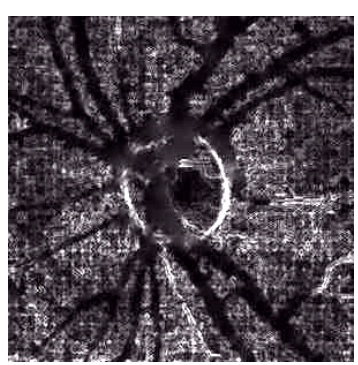

(a)

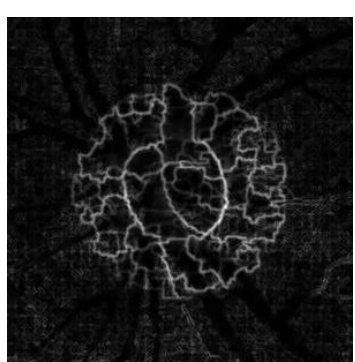

(b)

Figure 3.35: Probability density function: (a) Gradient image $\varrho\left(z_{1}^{\prime \prime}\right)$ and (b) Probability density function of contours using 15 simulations and 100 internal markers.

Finally, a last marker-controlled watershed is applied to $\rho\left(z_{1}^{\prime \prime}\right)(\mathbf{x})$ using a new set of markers $f_{m^{\prime}}$, giving rise to the image $\Pi\left(W S\left(\rho\left(z_{1}^{\prime \prime}\right) f_{m^{\prime}}\right)\right)$, which is shown in Figure 3.36(b), where the different obtained regions $\Pi$ can be appreciated. In this case, stratified markers are employed instead of random markers as shown Figure 3.36(a). The reason for using this type of markers is to make sure that every pixel within the area in question belongs to a watershed region. Due to the fact that there are markers located outside the OD, not all regions obtained by the watershed transformation are wanted. If each region of $\Pi\left(W S\left(\rho\left(z_{1}^{\prime \prime}\right) f_{m^{\prime}}\right)\right)$ is named as $\Pi_{i}$, the next stage of the proposed method is to discriminate which $\Pi_{i}$ are significant and which ones are not and should be filtered out. 


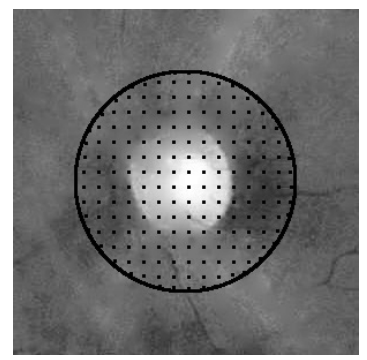

(a)

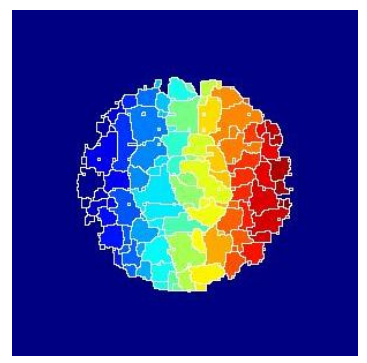

(b)

Figure 3.36: Last marker-controlled watershed: (a) Stratified markers, and (b) Watershed regions $\Pi\left(W S\left(\rho\left(z_{1}^{\prime \prime}\right)_{f_{m^{\prime}}}\right)\right)$.

\section{Region discrimination}

The discrimination between the significant and non-significant regions is based on the average intensity of the region. The value of each region will be equal to

$$
\mu_{\Pi_{i}}=\frac{1}{N_{i}} \sum_{\mathbf{x} \in \Pi_{i}} z_{1}^{\prime \prime}(\mathbf{x})
$$

being $N_{i}$ the number of pixels of the corresponding region $\Pi_{i}$ and $z_{1}^{\prime \prime}$ the inpainted image. Figure $3.37(\mathrm{a})$ represents the image $\mu_{\Pi}$ where the intensity of each region is equal to $\mu_{\Pi_{i}}$. The regions belonging to the optic disk will be light regions around darker regions therefore the residue of a close-hole operator is calculated on $\mu_{\Pi}$ to obtain the regions that accomplish this condition (Figure 3.37(c)). Afterwards, a threshold is applied on the resulting image to select the valid regions. This operation leads to the final OD segmentation (Figure 3.37(d)). The value of the threshold is $u=m-2 s$, being $m$ and $s$ the mean and the standard deviation of the residue of the close-hole operator. 


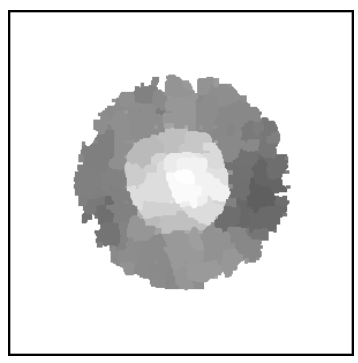

(a)

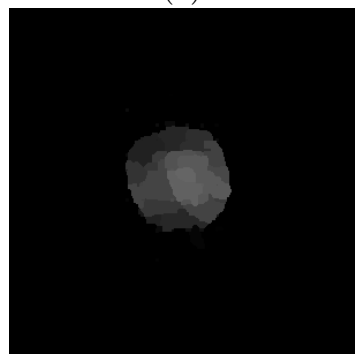

(c)

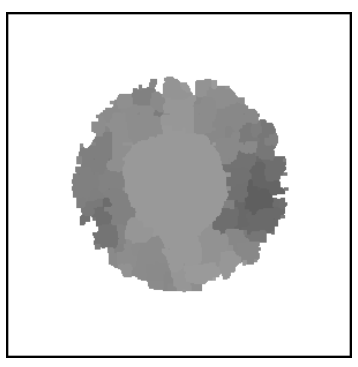

(b)

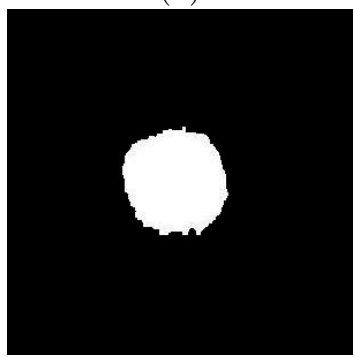

(d)

Figure 3.37: Region discrimination: (a) Average intensity of the watershed regions $\mu_{\Pi}$, (b) Close-hole operator, (c) Residue of close-hole operator, and (d) Thresholding.

\subsubsection{Post-processing}

Once the region of interest is obtained, the result must be fitted to eliminate false contours, which are detected due generally to the blood vessels that pass through the OD. The inpainting technique was performed to remove most of them, as previously mentioned, however some irregularities can still be appreciated in the final region contour (Figure 3.38(a)).

In this work, the OD-contour is estimated as a circle in the same way that in (Aquino et al., 2010; Niemeijer et al., 2009; Lalonde et al., 2001) although an elliptical shape could also be chosen. The main reason to fit the OD by a circle is because this algorithm will 
later be used to establish a zone of the retina concentric to the ODmargin according to a standard protocol with the aim to perform vessel diameter measurements (Wong et al., 2004). The fit is performed by means of Kasa's method (Corral and Lindquist, 1998) which lets calculate the center and the radius of the circle that better is adapted to a binary region through least squares. Figure 3.38 shows the contour of the final region obtained by the proposed method and its circular approximation.

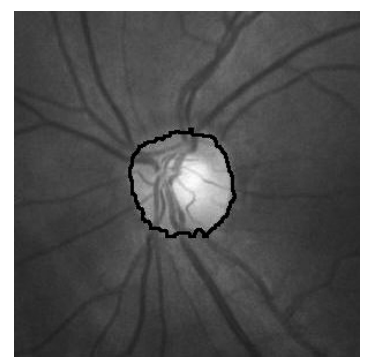

(a)

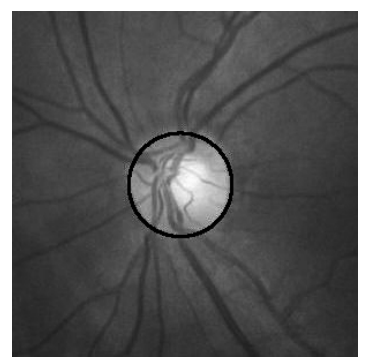

(b)

Figure 3.38: OD post-processing: (a) Contour of the obtained region, and (b) Circular approximation of the OD contour.

\subsection{Application to pathology detection: Hy- pertensive risk}

Most attempts to automate the process of interpretation of retinal vascular imaging are focused on a specific disease, diabetic retinopathy, a disease of high incidence and a significant risk of blindness that occupies a very important part of the medical-surgical activity of the ophthalmologic resources (Goatman et al., 2011; Abràmoff et al., 2010). In some of these studies, it was possible to relate the evolution of the disease and the positive or negative response to treatment with retinal vessel caliber (Nguyen et al., 2007; Rogers et al., 2008; Cheung and Wong, 2008). However, there is not too much experience in the use of 
these methods to evaluate other types of vascular pathology although fundus photography allows also the determination of cardiovascular risk factors (Abràmoff et al., 2010). Vascular changes produced in systemic diseases usually induce particular modifications in the vessels, such as changes in the angle of intersection between arteries and veins, and changes in the vessel calibers, but the existing solutions only focus on determining the caliber changes (Ruggeri et al., 2007; Muramatsu et al., 2010; Tramontan et al., 2008; Niemeijer et al., 2011; Ortega et al., 2010). Based on these facts, a system capable of detecting the retinal vessels and measuring several geometrical properties from a fundus image was developed. The goal of this software is to assist ophthalmologists in diagnosis and disease prevention, helping them to establish objective relations between the different vessels, to determine cardiovascular risk or other diseases where the vessels can be altered, as well as to monitor the pathology progression and response to different treatments. This tool was applied in a clinical study in order to evaluate sensitivity, specificity and reproducibility of the developed system to discriminate between a normal vascularization and cardiovascular pathology in contrast to the opinion of an expert ophthalmologist obtained through visual inspection of the fundus image.

The computer-aided diagnosis software presented in this section is based on digital fundus image processing for the determination of hypertensive risk. In general, systemic diseases produce vascular changes in our blood system and usually induce specific modifications in the retinal vessels; hence, the system is focused on vessel detection and the measurement of some of their features to characterize any retinal morphological change. So, the main stages involved in the system are: firstly, vessel segmentation process; secondly, retinal tree labelling to identify the pixels that correspond to each vessel and the existing relationship between the branches; and thirdly, performing characteristic measurements on the retinal tree, using the previous information, to quantify significant changes in the vascular network. These data will be used to classify an image as belonging to a healthy subject or to other with cardiovascular risk. The mentioned stages can 
be observed in the block diagram of the Figure 3.39 where the entire image processing carried out by the proposed system is illustrated.

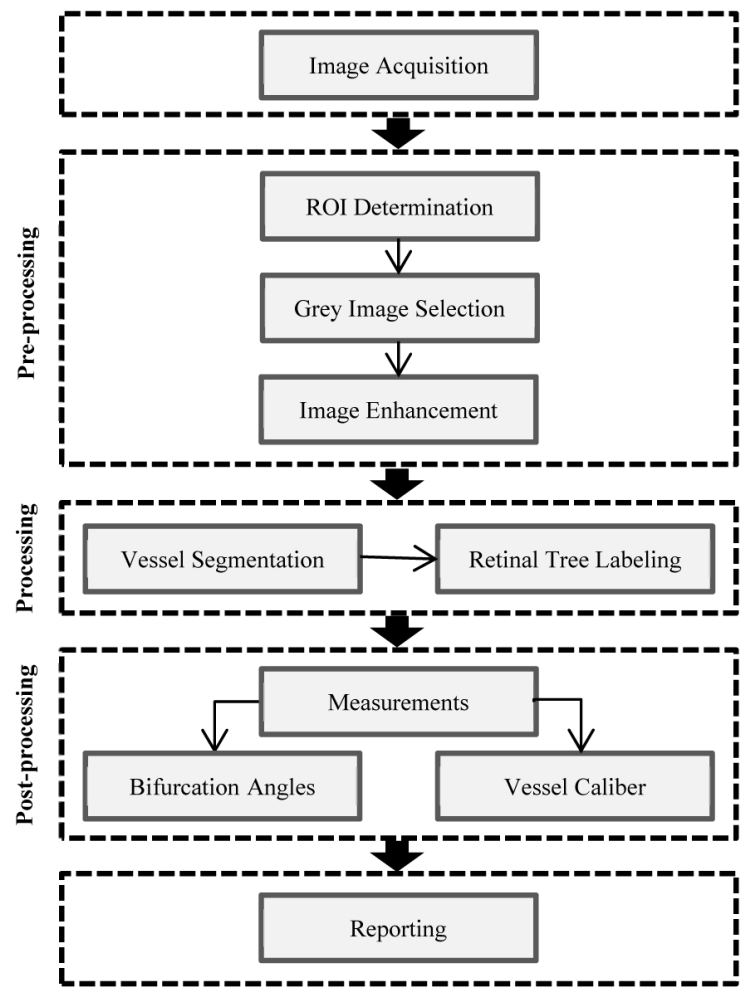

Figure 3.39: Block diagram of the presented tool.

\subsubsection{Pre-processing}

Generally, based on a standard protocol, the measurement of retinal vessel calibers is focused on a specific region of interest (ROI) of the fundus image (Wong et al., 2004; Knudtson et al., 2003; Hubbard et al., 1999). This area is concentric to the optic disk and it is related to its diameter. So, for that reason, in first place, the proposed system detects 
the optic disk (OD) in an automatic way in order to be able to determine the ROI where all measures will be performed. The method used for the extraction of the optic disk is explained in the previous section (Morales et al., 2013).

Once the ROI is established, although original fundus images are RGB images, the system is only drawn on the green band because this band provides an improved visibility of retinal blood vessels. Afterwards, an image enhancement (Walter and Klein, 2002) is applied to improve even more, if it is possible, their visibility (Equation 3.11).

\subsubsection{Processing}

The segmentation method used by the system is based on mathematical morphology, curvature evaluation and k-means clustering for the detection of a vascular tree (Morales et al., 2012) that is explained in Subsection 3.3.1.

After vessel detection, the vessels must be labelled. Retinal vascular tree labelling is focused on obtaining the skeleton of the vascular tree, detecting significant points and a tracking process (Morales et al., 2012). They are necessary steps to perform later the desired measures.

The skeleton of the vascular tree is obtained by a thinning process from the segmented binary image (Soille, 2003). Next, a pruning process is applied to eliminate possible spurs (Walter, 2003). Significant points (terminal, bifurcation and crossing points) are detected by the method proposed in Section 3.4.

The tracking purpose is to analyze the direction of every pixel of the skeleton and to identify which pixels belong to each branch. The method used is proposed in (Martinez-Perez et al., 2002). The algorithm is able to specify the direction of any skeleton point from a starting point and, therefore, it lets identify the parent and daughter branches.

Note that the method used for vessel segmentation and the later 
skeletonization could be replaced by the algorithm for vessel centerline extraction presented in Subsection 3.3.2.

\subsubsection{Post-processing}

Certain geometric measurements of blood vessels can help to establish whether they have undergone morphological changes over time and facilitate disease diagnosis. The remaining parameters were chosen due to the fact that they have particular interest for the early hypertension detection:

Bifurcation angle: Angle formed by the daughter branches for each bifurcation point. The branches are fitted for straight lines by leastsquares into a circular window centered on these points and then the angle $\alpha$ between them is calculated as

$$
\alpha=\arccos \left(\frac{\left|u_{1} \cdot v_{1}+u_{2} \cdot v_{2}\right|}{\sqrt{u_{1}^{2}+u_{2}^{2}} \cdot \sqrt{v_{1}^{2}+v_{2}^{2}}}\right),
$$

being $\mathbf{u}=\left(u_{1}, u_{2}\right)$ and $\mathbf{v}=\left(v_{1}, v_{2}\right)$ the direction vectors of these lines. See Figure 3.40 and 3.41.

Vessel caliber: It is estimated as two times the average of the distance transform value (Soille, 2003) on the skeleton points of the corresponding vessel. The distance transform is an operator applied to binary image. The result of the transform is a gray-level image whose intensity represents the distance from each pixel to the closest boundary. So that the caliber of a vessel $V_{i}$ is

$$
C_{V_{i}}=\frac{1}{N} \sum_{p \in S_{i}} d_{V_{i}}(p)
$$

being $d_{V_{i}}$ the distance transform of the vessel $V_{i}, S_{i}$ the skeleton of this 


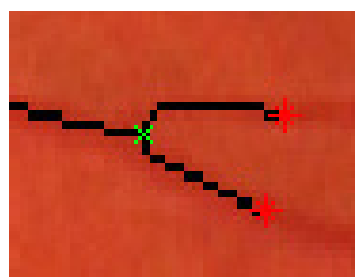

(a)

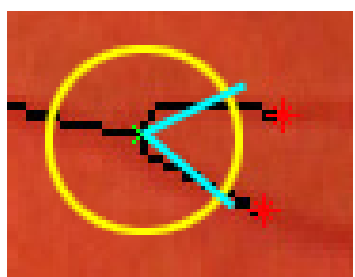

(b)

Figure 3.40: Bifurcation angle measurement: (a) Vessel bifurcation, and (b) Measurement process. A circular window (yellow) is centered on the bifurcation point (green cross) and the daughter branches are fitted for straight lines (cyan). The angle between these lines is directly calculated from their direction vectors.
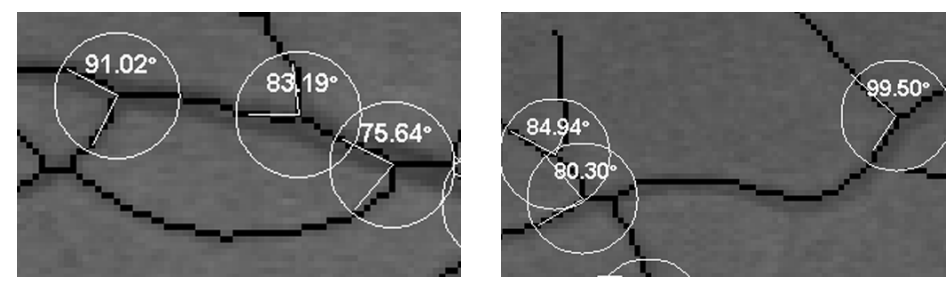

Figure 3.41: Bifurcation angles measured on two different image areas.

vessel and $N$ the number of pixels of $S_{i}$. Figure 3.42 shows a vessel binary mask and its corresponding distance transform.

As a summary, Figure 3.43 includes the main stages of the image processing performed by the developed software: ROI determination, vessel segmentation and angle and caliber measurement. 


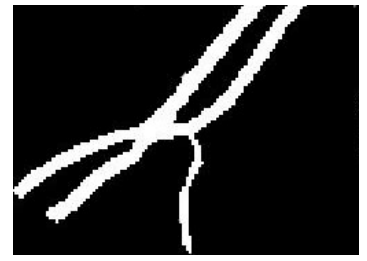

(a)

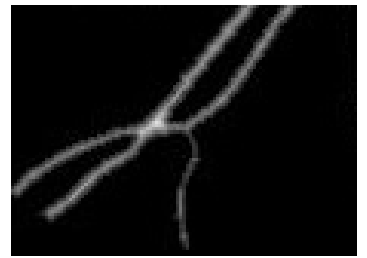

(b)

Figure 3.42: Vessel caliber measurement: (a) Vessel binary mask, and (b) Distance transform.

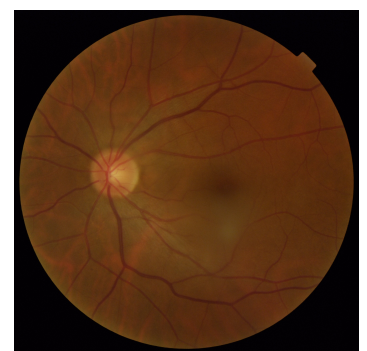

(a)

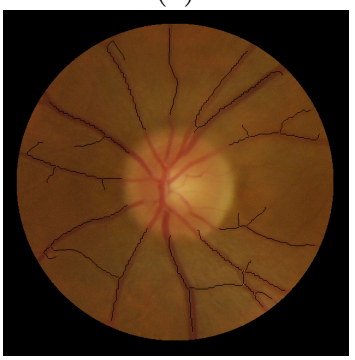

(c)

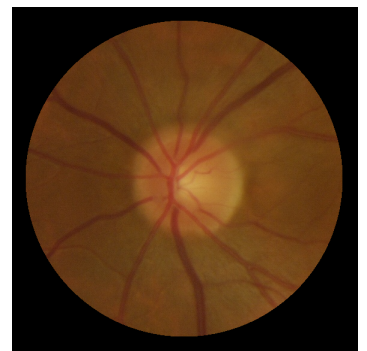

(b)

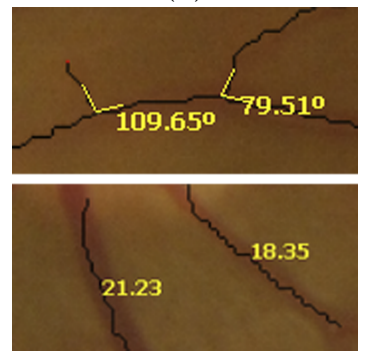

(d)

Figure 3.43: Image processing performed by the developed software: (a) Original fundus image, (b) ROI determination, (c) Vessel segmentation, (d) Bifurcation angles (up) and vessel calibers (down). 


\subsection{Results}

In this section, the performance of the methods presented in this chapter is detailed along with a description of the databases used for that purpose.

\subsubsection{Material}

Different public and private databases were used to validate the methods proposed in this chapter. Next, a description of each of them is included.

\section{DRIVE}

DRIVE (Digital Retinal Images for Vessel Extraction) (Staal et al., 2004) is a public database established to enable comparative studies on retinal vessel segmentation. ${ }^{1}$ The database is formed by 40 retinal images (768 x 584) belonging to diabetic subjects between 25-90 years of age. 33 do not show any sign of diabetic retinopathy and 7 show signs of mild early diabetic retinopathy.

For each image, a mask image that delineates the field of view is provided as well as manual segmentations of the blood vessels. The images are divided into a training and a test set, both of 20 images. For the training images, only a single manual segmentation is available. For the test cases, two manual segmentations are included.

\section{STARE}

STARE (STructured Analysis of the Retina) database (Hoover et al., 2000) is designed for blood vessel segmentation. ${ }^{2}$ This dataset consists

\footnotetext{
${ }^{1}$ www.isi.uu.nl/Research/Databases/DRIVE/

2 ww. ces.clemson. edu/ ahoover/stare/probing/index.html
} 
of 20 retinal images $(700 \times 605)$. For each image, vessel network is hand labelled by two experts.

\section{DRIONS}

DRIONS (Digital Retinal Images for Optic Nerve Segmentation) Database (Carmona et al., 2008) is a public database for benchmarking optic nerve head segmentation from retinal images. ${ }^{3}$ The database consists of 110 retinal images $(600 \times 400)$ whose optic disk is manually segmented by two specialists. The mean age of the patients was 53.0 years (S.D. 13.05), with $46.2 \%$ male and $53.8 \%$ female and all of them were Caucasian ethnicity. $23.1 \%$ patients had chronic simple glaucoma and $76.9 \%$ eye hypertension. Some of the 110 images contain visual characteristics related to potential problems that may distort the detection process of the contour of the optic disk: light artefacts (3 images), some of rim blurred or missing (5 images), moderate peripapillary atrophy (16 images), concentric peripapillary atrophy/artefacts (20 images), and strong pallor distractor (6 images).

\section{DIARETDB1}

DIARETDB1 database (Kauppi et al., 2007) is a public database for diabetic retinopathy detection. ${ }^{4}$ The database is formed by 89 color fundus images $(1500 \times 1152) .84$ of them contain at least mild nonproliferative signs (microaneurysms) of the diabetic retinopathy, and five are considered as normal which do not contain any signs of the diabetic retinopathy according to all experts who participated in the evaluation. Four medical experts marked the areas related to the microaneurysms, hemorrhages, and hard and soft exudates.

\footnotetext{
${ }^{3}$ www.ia.uned.es/ ejcarmona/DRIONS-DB.html

${ }^{4}$ www.it.lut.fi/project/imageret/diaretdb1/
} 


\section{MESSIDOR}

MESSIDOR database, kindly provided by the Messidor program partners, was established to facilitate studies of diabetic retinopathy detection. $^{5}$ It contains 1200 eye fundus color images of the posterior pole. The images were captured using 8 bits per color plane at different resolutions (1440 x 960, $2240 \times 1488$ and $2304 \times 1536)$. 800 images were acquired with pupil dilation and 400 without dilation. For each image, two diagnosis were provided: retinopathy grade and risk of macular edema.

\section{ONHSD}

ONHSD (Optic Nerve Head Segmentation Dataset) contains 99 fundal images (640 x 480) taken from 50 patients randomly sampled from a diabetic retinopathy screening program; 96 images have discernable optic nerve head (Lowell et al., 2004). The subjects are from various ethnic backgrounds; 19 have type 2 diabetes mellitus, while the diabetes status was unavailable for the remaining 31 . In this database there is considerable quality variation in the images, with many characteristics that can affect segmentation algorithms. The optic nerve head center is marked up a clinician, and four clinicians marked the optic nerve head edge where it intersects with radial spokes (at 15 angles) radiating from the nominated center. ${ }^{6}$

\section{FOMDB}

The Fundación Oftalmológica del Mediterráneo (Spain) provided us a private dataset of 67 fundus images (2048 x 1536) for hypertensive risk determination. The images belong to 67 subjects between 33 and 73 years old. Among them, a group without previous known pathology and a group of hypertensive patients were established. The first group was

\footnotetext{
${ }^{5}$ http://messidor.crihan.fr/download-en.php

${ }^{6}$ http: //reviewdb. Iincoln.ac.uk/ImageDatasets/ONHSD . aspx
} 
formed by 38 healthy controls and the second group by 29 hypertensive patients previously diagnosed and treated or not for more than 5 years of evolution. Both groups were established based on the disease history of their members.

\subsubsection{Retinal vessel segmentation}

To validate the vessel segmentation, the results of the method of Subsection 3.3.1 were compared with the hand-segmented images from DRIVE database (Staal et al., 2004). The first-observer images were taken as reference (gold standard) to calculate similarity degree between them and our segmentation.

The performance of the method was evaluated based on three concepts: accuracy $(A c)$ and true positive $(T P F)$ and false positive $(F P F)$ fractions. Accuracy $(A c)$ is determined by the sum of correctly classified pixels as vessel and non-vessel divided by the total number of pixels in the image. True positive fraction $(T P F)$, also known as sensitivity, is established by dividing the correctly classified pixels as vessel by the total number of vessel pixels in the gold standard. False positive fraction $(F P F)$ is calculated by dividing the misclassified pixels as vessel by the total number of non-vessel pixels in the gold standard. In Table 3.1 these results can be observed. The images manually segmented by the second observer were also compared with the gold standard in order to note that there are differences between both experts. In summary, the average values obtained by the proposed method were: $A c=0.9417, T P F=0.6570$ and $F P F=0.0166$. Table 3.2 displays the true positive/negative and false positive/negative pixels used to calculate the measures of the Table 3.1. 
Table 3.1: Comparison between the results of the method proposed in Subsection 3.3.1 and the $2^{\text {nd }}$ observer of DRIVE database (average values and standard deviations) regarding the gold standard.

\begin{tabular}{lccc}
\hline & Ac & TPF & FPF \\
\hline Proposed method & $0.9417(0.0076)$ & $0.6570(0.0668)$ & $0.0166(0.0093)$ \\
$2^{n d}$ observer & $0.9473(0.0048)$ & $0.7757(0.0596)$ & $0.0275(0.0083)$ \\
\hline
\end{tabular}

Table 3.2: True positives/negatives and false positives/negatives (TP, TN, FP and FN). Average values and standard deviations.

\begin{tabular}{lcccc}
\hline & TP & TN & FP & FN \\
\hline Proposed & 18,945 & 194,740 & 3,284 & 9,938 \\
method & $(2,309)$ & $(3,557)$ & $(1,842)$ & $(2,294)$ \\
$2^{n d}$ observer & 22,374 & 192,570 & 5,453 & 6,508 \\
& $(2,389)$ & $(3,083)$ & $(1,685)$ & $(1,895)$ \\
\hline
\end{tabular}

Figure 3.44 depicts some vessel segmentation results. The first two rows show good segmentation results where only the very thin vessels are not detected. In the last two rows, in addition to the non-detection of the very thin vessels, a bit of over-segmentation can be observed in some areas of the images.

On the other hand, in (Niemeijer et al., 2004) a study about the accuracy of different vessel segmentation methods was carried out on the same database. This study compared several approaches: matched filter (Chaudhuri et al., 1989), scale-space analysis and region growing (Martinez-Perez et al., 1999), mathematical morphology and curvature estimation (Zana and Klein, 2001), verification-based local thresholding (Jiang and Mojon, 2003) and pixel classification (Niemeijer et al., 2004). Table 3.3 shows a comparison between the aforementioned methods and ours. Our method achieved a higher accuracy ratio and its false positive fraction is, even, lower than the second observer fraction. 

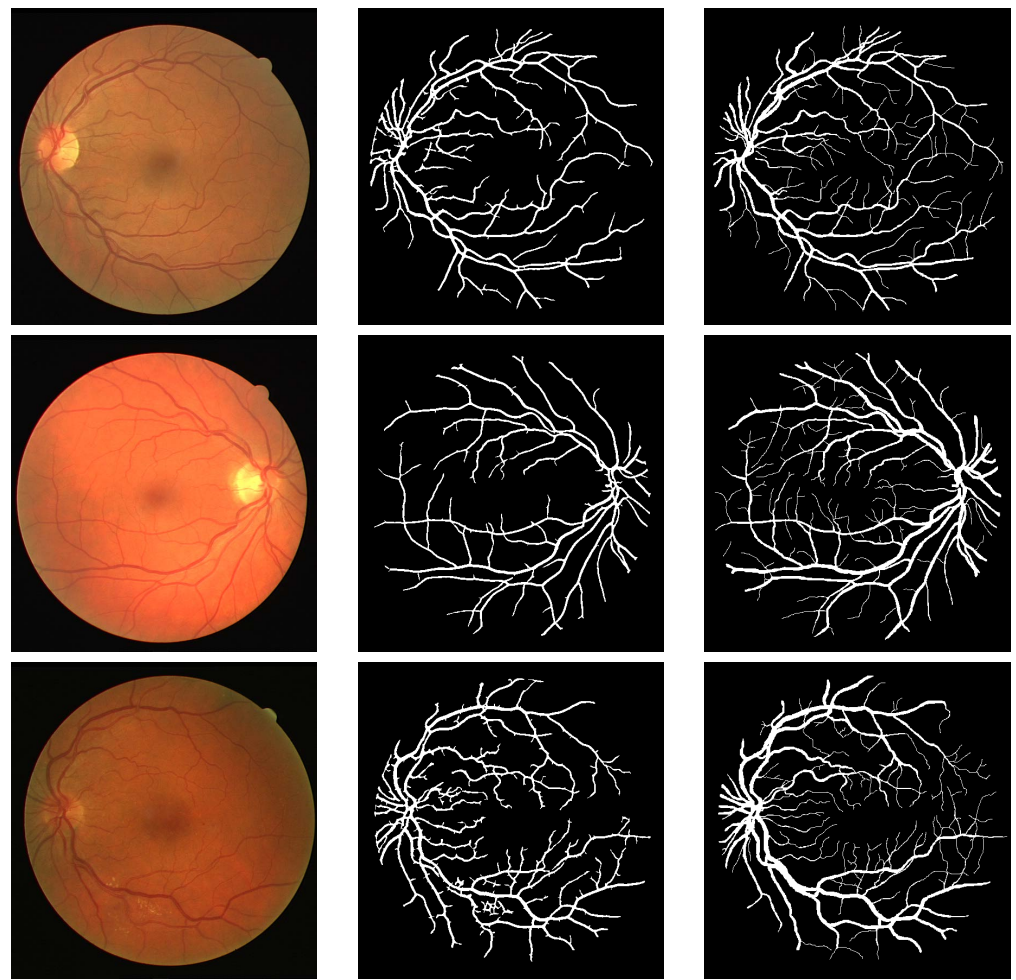

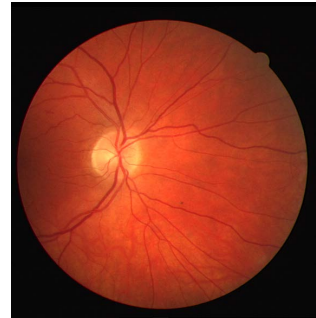

(a)

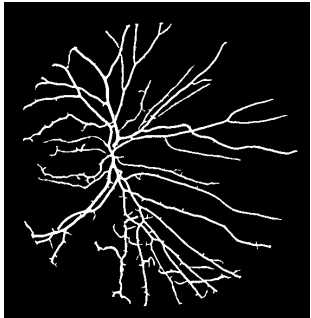

(b)

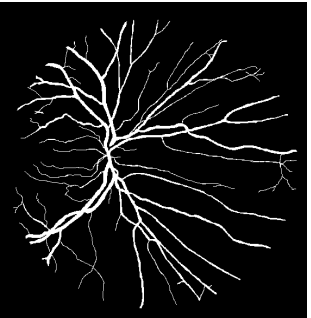

(c)

Figure 3.44: Some vessel segmentation results on DRIVE database: (a) Original fundus images, (b) Results of the method proposed in Subsection 3.3.1, and (c) Ground truth images. 
Table 3.3: Accuracy (average and standard deviation) of several methods on DRIVE database.

\begin{tabular}{lc}
\hline & Ac \\
\hline Proposed method & $0.9417(0.0076)$ \\
$2^{\text {nd } \text { observer }}$ & $0.9473(0.0048)$ \\
Niemeijer et al. (Niemeijer et al., 2004) & $0.9416(0.0065)$ \\
Zana and Klein (Zana and Klein, 2001) & $0.9377(0.0077)$ \\
Jiang and Mojón (Jiang and Mojon, 2003) & $0.9212(0.0076)$ \\
Martínez-Pérez et al. (Martinez-Perez et al., 1999) & $0.9181(0.0240)$ \\
Chaudhuri et al. (Chaudhuri et al., 1989) & $0.8773(0.0232)$ \\
\hline
\end{tabular}

In addition to improving the accuracy ratio, it must be stood out that the optic disk edge is not detected as vessel unlike the most edge detection methods. Figure 3.45 shows our method along with the proposed in (Martinez-Perez et al., 2007). The non-detection of this feature is crucial to avoid multiple errors in any tracking process.

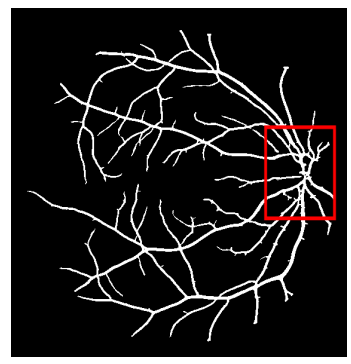

(a)

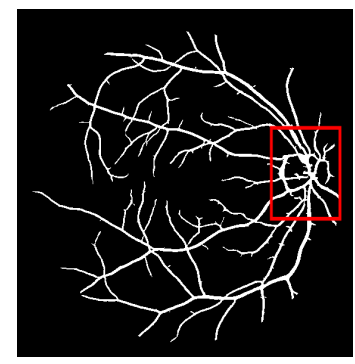

(b)

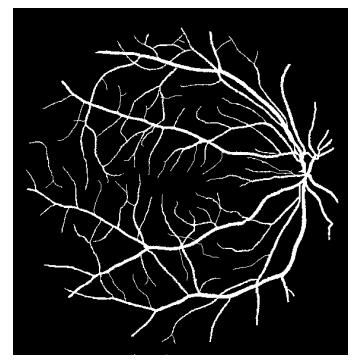

(c)

Figure 3.45: Vessel segmentation comparison: (a) Method proposed in Subsection 3.3.1, (b) Method proposed in (Martinez-Perez et al., 2007) and (c) Manually segmented image belonging to the DRIVE database.

After the study performed by Niemeijer et al. (Niemeijer et al., 2004), other methods about retinal vessel segmentation were published. As far the author knows, the best results on DRIVE database were presented by Mendonça and Campilho (Mendonça and Campilho, 2006) 
and Ricci and Perfetti (Ricci and Perfetti, 2007). Although these works achieved a slightly higher accuracy than the proposed method, $A c=0.9463$ and $A c=0.9595$, respectively; the border of the optic disk was still detected as vessel.

To evaluate the effect of the input image in the segmentation method, different tests were performed. The use of the red and green component and PCA were compared, being the green component which obtained the best results as can be appreciated in Figure 3.46.

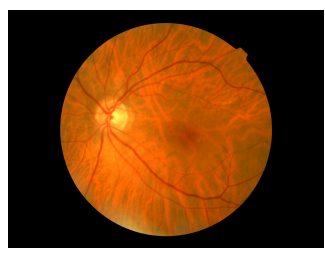

(a)

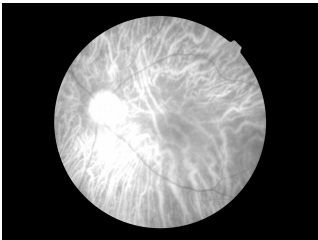

(b)

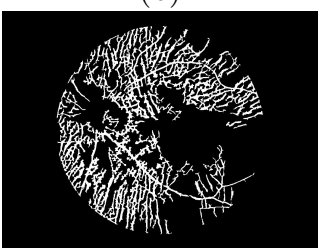

(e)

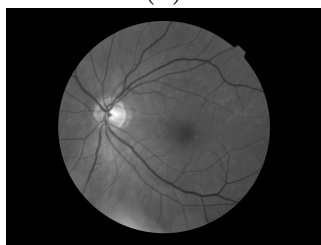

(c)

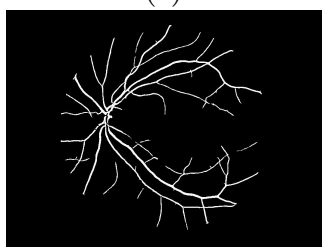

(f)

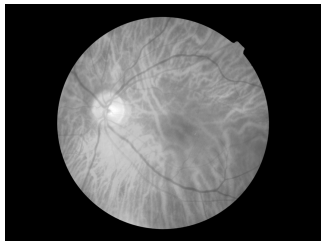

(d)

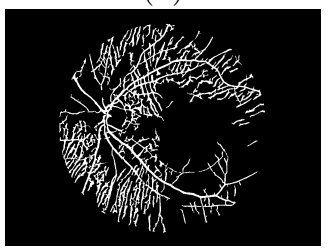

(g)

Figure 3.46: Effect of the input image in the segmentation method: (a) Original RGB image, (b)Red component, (c) Green component, (d) First component of PCA, (e) Result of the method proposed in Subsection 3.3.1 on the red component, (f) Result of the method proposed in Subsection 3.3.1 on the green component and $(g)$ Result of the method proposed in Subsection 3.3.1 on the first component of PCA. 


\subsubsection{Retinal vessel centerline}

The validation of the vessel centerline extraction method, that was presented in Subsection 3.3.2, was carried out on 2 public databases widely used: DRIVE (Staal et al., 2004) and STARE (Hoover et al., 2000). Although, in both databases, manual segmentations are included, the complete vasculature was detected, not only the vessel centerline which is the goal of our work. For that reason, the homotopic skeleton (Soille, 2003) associated to the manual segmentations was obtained for future comparisons. In Figure 3.47, the results of the proposed method on two images of DRIVE and STARE databases can be observed.

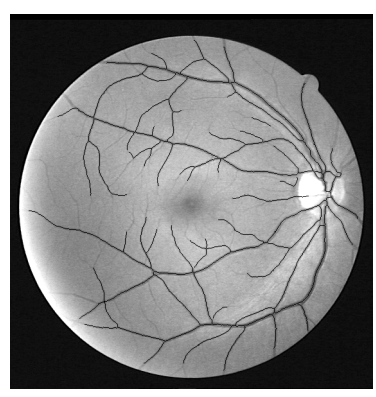

(a)

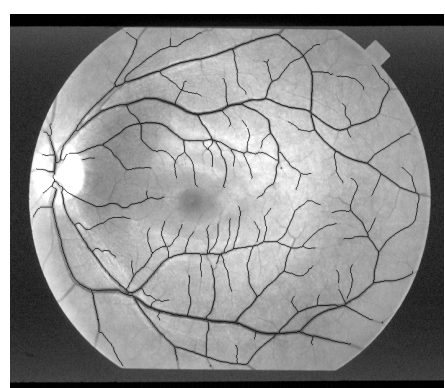

(b)

Figure 3.47: Skeleton results of the method proposed in Subsection 3.3.2: (a) DRIVE image ('19_test') and (b) STARE image ('im0255').

The validation was performed in two ways. One of them is based on comparing the results of this work with methods that first segment the vessels and after perform a skeletonization process and the other compares it with algorithms that directly obtain the skeleton.

On the one hand, regarding methods that require a previous segmentation, the presented algorithm was compared with two methods previously published. The first compared method uses the local maxima over scales of the magnitude of the gradient and the maximum principal curvature of the Hessian tensor in a multiple pass region growing 
procedure (Martinez-Perez et al., 2007). The other compared method is that explained in Subsection 3.3.1. As mentioned before, both methods of the Subsection 3.3.1 and 3.3.2 are based on mathematical morphology and curvature evaluation although the morphological operations used are different as well as the obtained result. In the same way as explained above, the homotopic skeleton was performed after the segmentation process in both cases. On the other hand, as for the methods that obtain directly the retinal vessel centerline, the analysis was focused on other two approaches based also on the watershed transformation (Walter and Klein, 2002; Bessaid et al., 2009). In Figures 3.48 and 3.49, the strengths and weaknesses of the proposed method can be observed in two examples of both databases.

Avoiding complete vessel segmentation supposes an improvement in the automatic fundus processing since the skeleton is not dependent of a previous stage and the computational cost is reduced by decreasing the number of required steps. Apart from this fact, it must be stressed that an important advantage of the proposed method is its performance in pathological images or with large changes in illumination, as was observed in Figures 3.47, 3.48 and 3.49. In those cases, the presented algorithm works properly and reduces over-segmentation problems which can be found in methods based on a previous segmentation (Martinez-Perez et al., 2007; Morales et al., 2012). With regard to other methods that obtain the skeleton in a direct way and use the watershed transformation (Bessaid et al., 2009; Walter and Klein, 2002) instead of the stochastic watershed, the proposed work achieves a more robust detection and decreases the number of spurs. Despite good results, it must be mentioned that the main disadvantage of the method is that some vessels can lose their continuity if some part of them is not detected and it should be corrected in a post-processing stage.

\subsubsection{Optic disk}

The performance of the OD-segmentation method proposed in Section 3.5 was evaluated based on different concepts. Jaccard's 


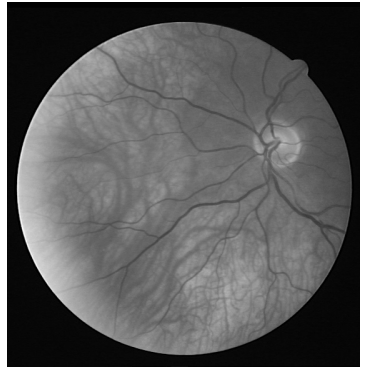

(a)

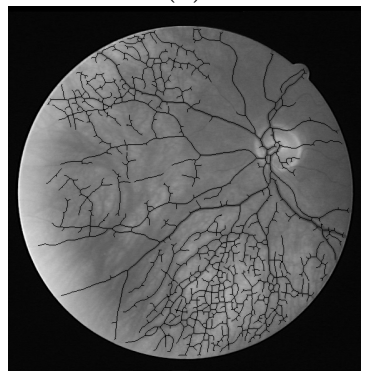

(d)

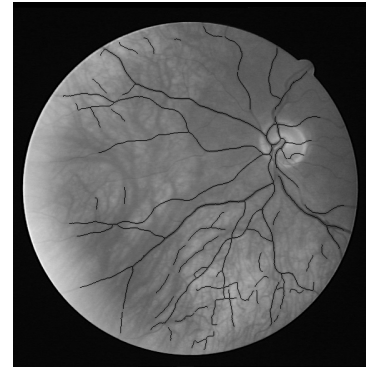

(b)

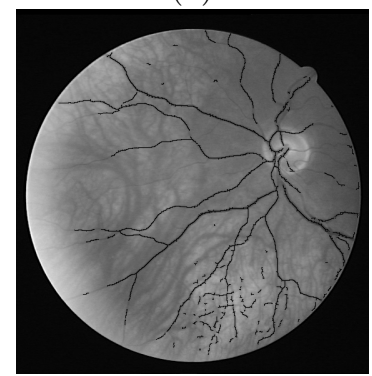

(e)

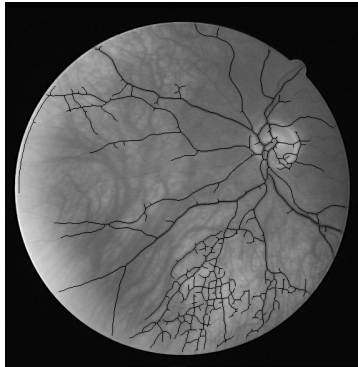

(c)

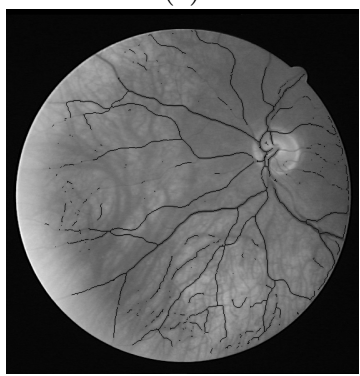

(f)

Figure 3.48: Comparison between different methods on DRIVE image ('23_training'): (a) Original image, (b) Method proposed in Subsection 3.3.2, (c) Martinez et al. method (Martinez-Perez et al., 2007), (d) Method presented in Subsection 3.3.1 (Morales et al., 2012), (e) Bessaid et al. method (Bessaid et al., 2009) and (f) Walter and Klein method (Walter and Klein, 2002).

(JC) and Dice's (S) coefficients describe similarity degree between two compared elements being equal to 1 when segmentation is perfect. Accuracy (Ac) is determined by the sum of correctly classified pixels as OD and non-OD divided by the total number of pixels in the image. True positive fraction (TPF) is established by dividing the correctly classified pixels as OD by the total number of OD pixels in the gold standard. False positive fraction $(\mathrm{FPF})$ is calculated by dividing the misclassified pixels as OD by the total number of non-OD pixels in the gold standard. Finally, in order to be able to compare with more other authors' works, another measure was calculated: the mean absolute 


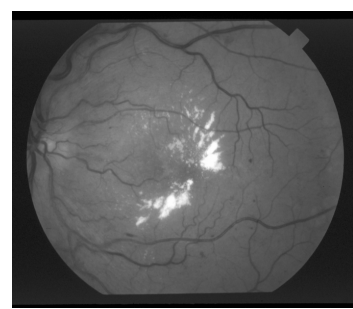

(a)

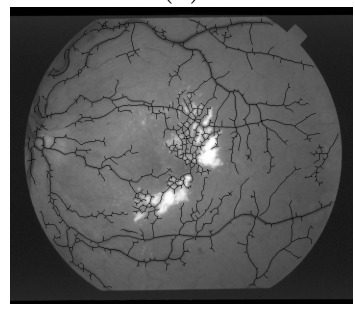

(d)

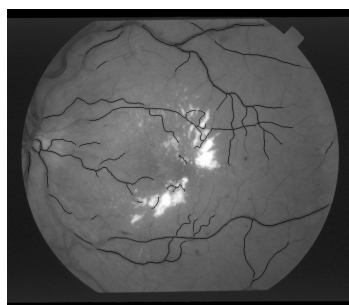

(b)

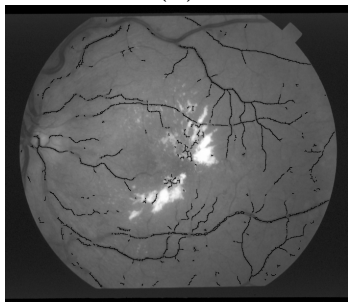

(e)

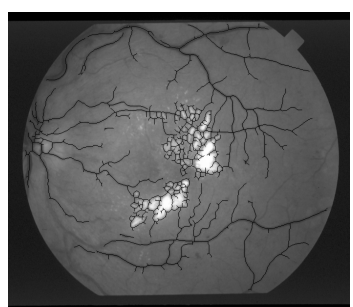

(c)

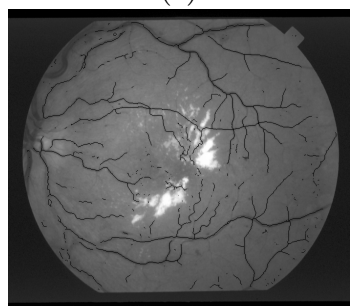

(f)

Figure 3.49: Comparison between different methods on STARE image ('im0001'): (a) Original image, (b) Method proposed in Subsection 3.3.2, (c) Martinez et al. method (Martinez-Perez et al., 2007), (d) Method presented in Subsection 3.3.1 (Morales et al., 2012), (e) Bessaid et al. method (Bessaid et al., 2009) and (f) Walter and Klein method (Walter and Klein, 2002).

distance (MAD) (Chalana et al., 1996), whose aim is to measure the accuracy of the OD-boundary,

$$
M A D(\mathcal{A}, \mathcal{B})=\frac{1}{2}\left\{\frac{1}{n} \sum_{i=0}^{n} d\left(\mathbf{a}_{i}, \mathcal{B}\right)+\frac{1}{n} \sum_{i=0}^{n} d\left(\mathbf{b}_{i}, \mathcal{A}\right)\right\}
$$

where $\mathcal{A}$ and $\mathcal{B}$ are two curves corresponding to the border of the segmented OD and the border of the OD considered as ground truth, respectively. They are represented as set of points $\mathcal{A}=\left\{\mathbf{a}_{1}, \mathbf{a}_{2}, \ldots, \mathbf{a}_{n}\right\}$ and $\mathcal{B}=\left\{\mathbf{b}_{1}, \mathbf{b}_{2}, \ldots, \mathbf{b}_{n}\right\}$ and $d\left(\mathbf{a}_{i}, \mathcal{B}\right)$ is the distance to the closest point for point $\mathbf{a}_{i}$ on the curve $\mathcal{B}$. 
In Table 3.4 the results on 5 public databases can be observed: DRIONS (Carmona et al., 2008), DIARETDB1 (Kauppi et al., 2007), DRIVE (Staal et al., 2004), MESSIDOR, and ONHSD (Lowell et al., 2004). Note that all these measures are indicators of the segmentation quality but in different way, i.e. a database can have a higher value of a measure than other database but this does not mean that this tendency must be followed by the rest. For example, the MAD can be equal in a case of under-segmentation and in other of over-segmentation if the distance between the boundary of our result and the ground truth (GT) is the same. As the OD is fitted by a circle, this situation may occur if our result and the ground truth were two concentric circles as shown in Figure 3.50. Although the MAD was equal, the rest of the measures could be different. Table 3.5 displays the true positive/negative and false positive/negative pixels used to calculate all measures of Table 3.4 .

Table 3.4: Results (average values and standard deviations) obtained by the method proposed in Subsection 3.5 on DRIONS, DIARETDB1, DRIVE, MESSIDOR, and ONHSD databases. Jaccard's (JC) and Dice's (S) coefficients, accuracy (Ac), true positive (TPF) and true negative fractions $(F P F)$, and mean absolute distance (MAD).

\begin{tabular}{|c|c|c|c|}
\hline & $\mathrm{JC}$ & $\mathrm{S}$ & Ac \\
\hline DRIONS & $0.8424(0.1174)$ & $0.9084(0.0982)$ & $0.9934(0.0051)$ \\
\hline DIARETDB1 & $0.8173(0.1308)$ & $0.8930(0.0913)$ & $0.9957(0.0039)$ \\
\hline DRIVE & $0.7163(0.1880)$ & $0.8169(0.1712)$ & $0.9903(0.0134)$ \\
\hline MESSIDOR & $0.8228(0.1384)$ & $0.8950(0.1056)$ & $0.9949(0.0050)$ \\
\hline \multirow[t]{2}{*}{ ONHSD } & $0.8045(0.1175)$ & $0.8867(0.0776)$ & $0.9941(0.0042)$ \\
\hline & $\mathrm{TPF}$ & $\mathrm{FPF}$ & MAD \\
\hline DRIONS & $0.9281(0.117$ & $0.0040(0.00$ & $4.1098(3.4684)$ \\
\hline DIARETDB1 & $0.9224(0.1058)$ & $0.0028(0.0029)$ & $9.6759(8.4836)$ \\
\hline DRIVE & $0.8544(0.1938)$ & $0.0061(0.0085)$ & $7.9981(9.9957)$ \\
\hline MESSIDOR & $0.9300(0.1239)$ & $0.0035(0.0041)$ & $5.8387(6.5215)$ \\
\hline ONHSD & $0.9310(0.1046)$ & $0.0043(0.0042)$ & $4.4826(3.0962)$ \\
\hline
\end{tabular}




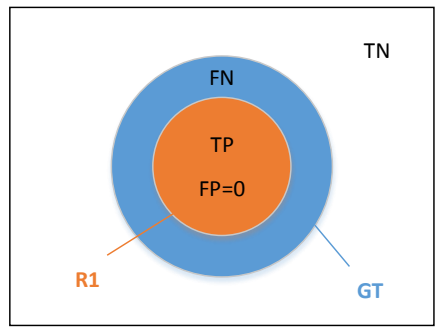

(a)

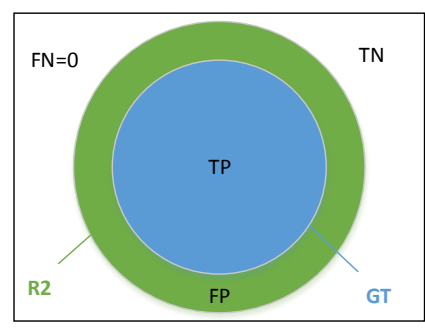

(b)

Figure 3.50: Sub and over-segmentation case: (a) Under-segmentation and (b) Over-segmentation. GT (in blue) is the ground truth in both cases and R1 (in orange) and R2 (in green) the result of our segmentation in each case. R1 is characterized by not having false positives $(F P=0)$ and $R 2$ by not having false negatives $(F N=0)$. Moreover, the number of true positives (TP) will be greater in (b) than in (a) in contrast to the true negatives (TN). Although the $M A D$ was equal for both cases, these variations could provoke that the rest of the measures were different.

Table 3.5: True positives/negatives and false positives/negatives (TP, TN, FP and FN). Average values and standard deviations.

\begin{tabular}{lcccc}
\hline & TP & TN & FP & FN \\
\hline DRIONS & $6,701(1,392)$ & $185,870(3,467)$ & $753(763)$ & $517(877)$ \\
DIARETDB1 & $4,448(875)$ & $243,970(1,087)$ & $681(705)$ & $380(542)$ \\
DRIVE & $4,394(1,252)$ & $220,380(2,867)$ & $1,340(1,869)$ & $869(1670)$ \\
MESSIDOR & $9,773(4,434)$ & $427,450(168,890)$ & $1,415(1,757)$ & $804(1,529)$ \\
ONHSD & $4,667(1,048)$ & $196,240(1,262)$ & $846(828)$ & $345(516)$ \\
\hline
\end{tabular}

On the one hand, in order to analyze the results on DRIONS database, the first observer images of this database were taken as reference (gold standard) to calculate similarity degree between them and our segmentation. Regarding MESSIDOR database, the OD rim of these 1200 images was hand segmented by the University of Huelva 
(Spain) and it is currently available online to facilitate performance comparison between different methods. ${ }^{7}$ Concerning ONHSD dataset, the average of the edges marked by the four experts was used to generate the reference images. On the other hand, neither DIARETDB1 nor DRIVE databases have the OD segmented publicly available. In those cases, we compared our results with the same ground truth used in (Welfer et al., 2010), where the contour of each image was labelled by four ophthalmologists, and then, only the mean of those contours is considered as ground truth.

Apart from quantifying the accuracy in the OD boundary detection (Table 3.4), the accuracy in the disk localization was also measured. The performance of the optic disk localization is shown in Table 3.6. An automatically detected OD was considered correct if there exist intersection between it and the OD marked manually.

Table 3.6: Success rate in the optic disk location.

\begin{tabular}{lc}
\hline & Location \\
\hline DRIONS & 100 \\
DIARETDB1 & 100 \\
DRIVE & 97.50 \\
MESSIDOR & 95.83 \\
ONHSD & 100 \\
\hline
\end{tabular}

Some examples of good, fair and bad OD segmentation results on the different databases analyzed are shown from Figure 3.51 to Figure $3.55)$.

\footnotetext{
${ }^{7}$ www.uhu.es/retinopathy/muestras.php
} 

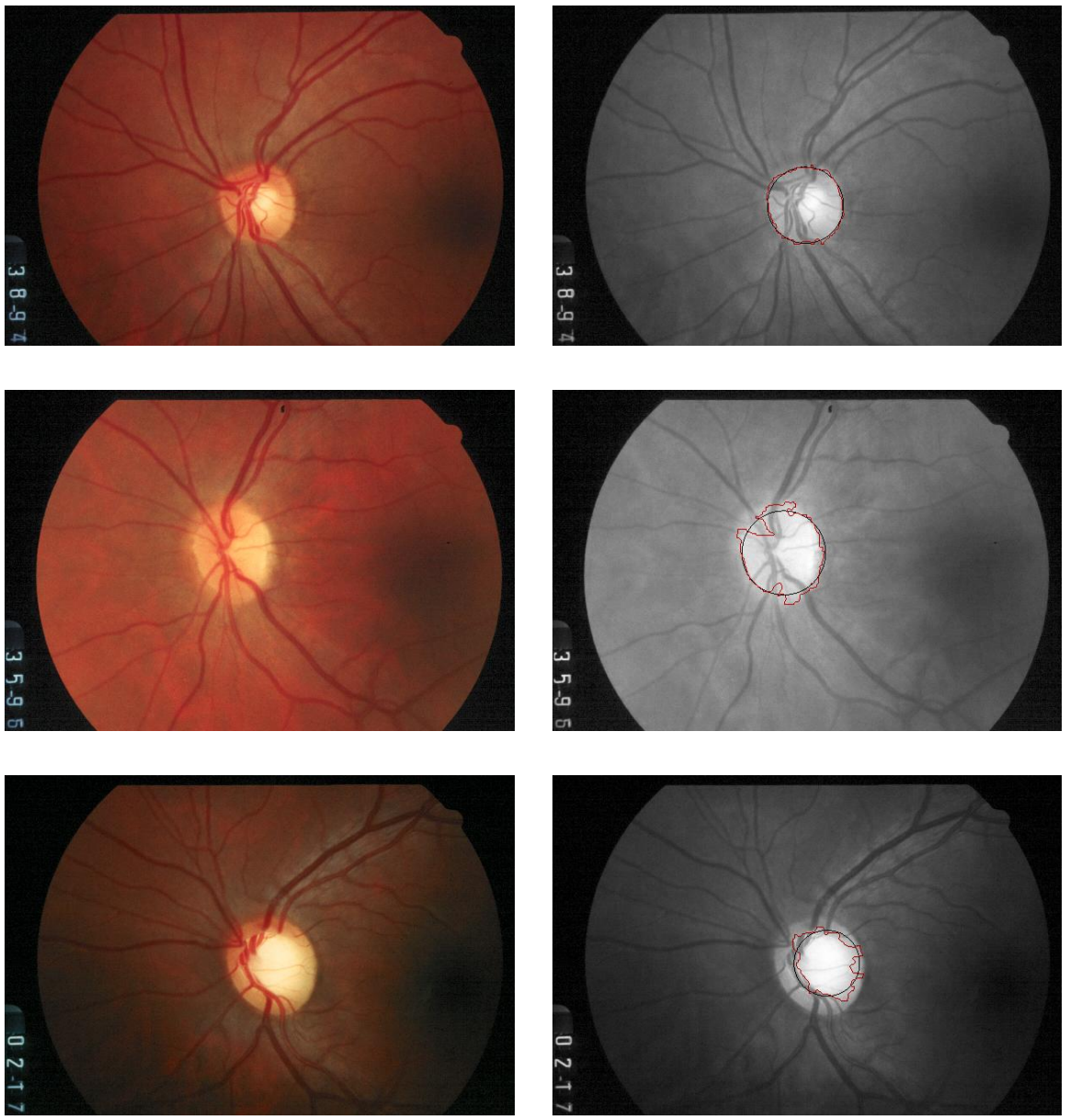

(a)

(b)

Figure 3.51: Some OD segmentation results on DRIONS database: (a) Original fundus images, (b) Results of the method proposed in Section 3.5 (OD contour in red and circular approximation of the OD contour in black). Top row: good segmentation. Middle row: fair segmentation. Bottom row: poor segmentation. 

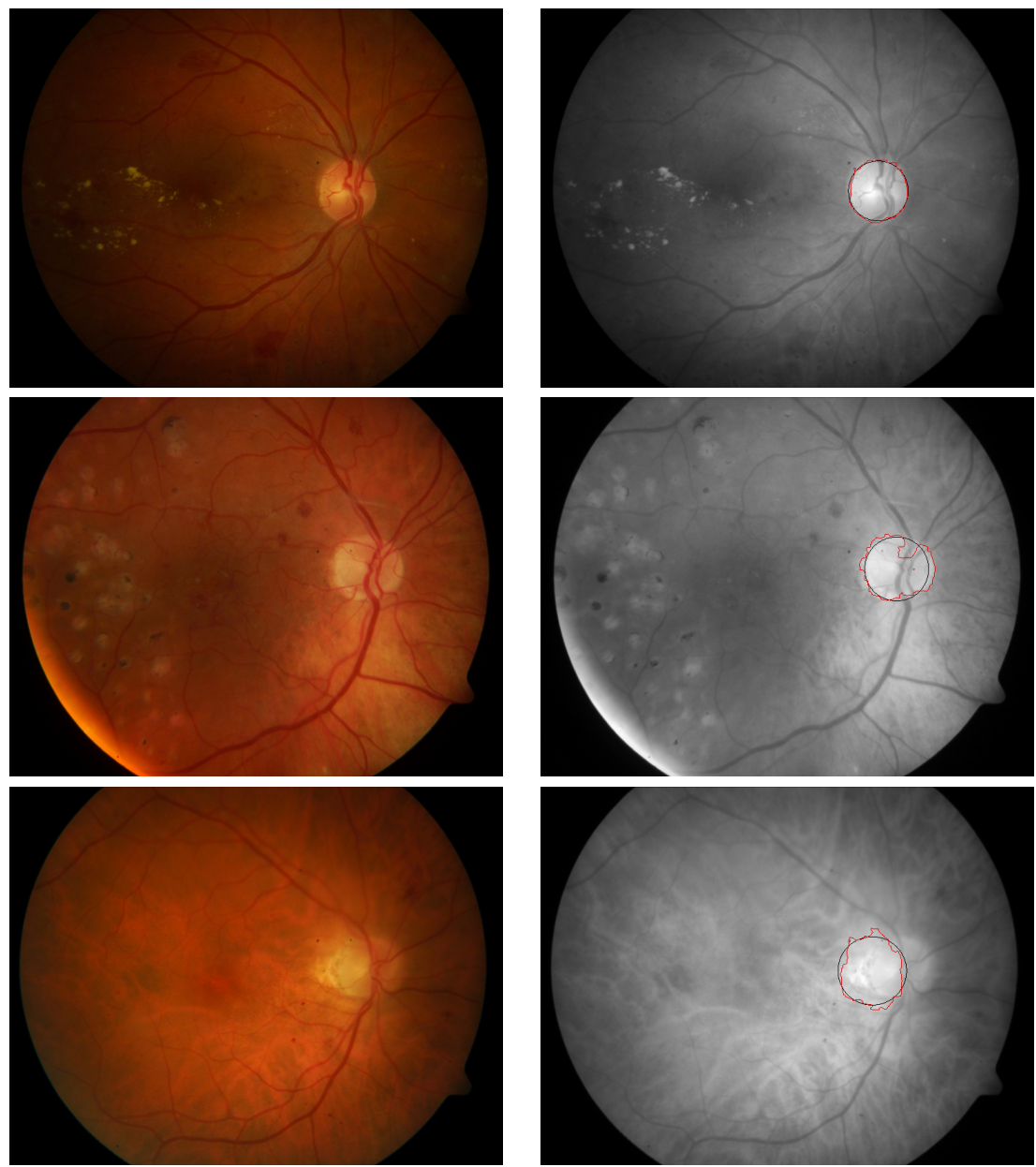

(a)

(b)

Figure 3.52: Some OD segmentation results on DIARETDB1 database: (a) Original fundus images, (b) Results of the method proposed in Section 3.5 (OD contour in red and circular approximation of the $O D$ contour in black). Top row: good segmentation. Middle row: fair segmentation. Bottom row: poor segmentation. 

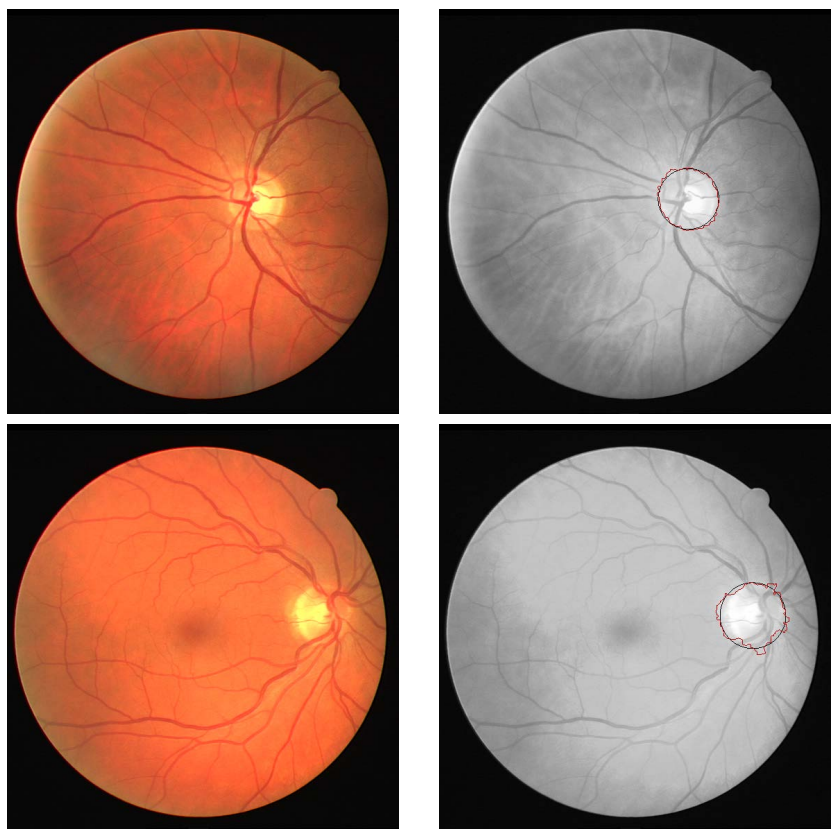

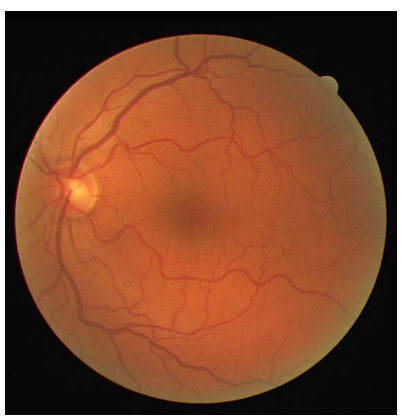

(a)

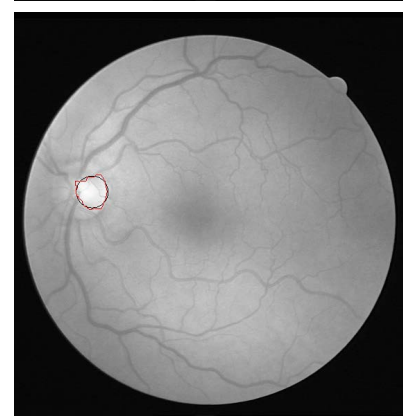

(b)

Figure 3.53: Some OD segmentation results on DRIVE database: (a) Original fundus images, (b) Results of the method proposed in Section 3.5 (OD contour in red and circular approximation of the OD contour in black). Top row: good segmentation. Middle row: fair segmentation. Bottom row: poor segmentation. 

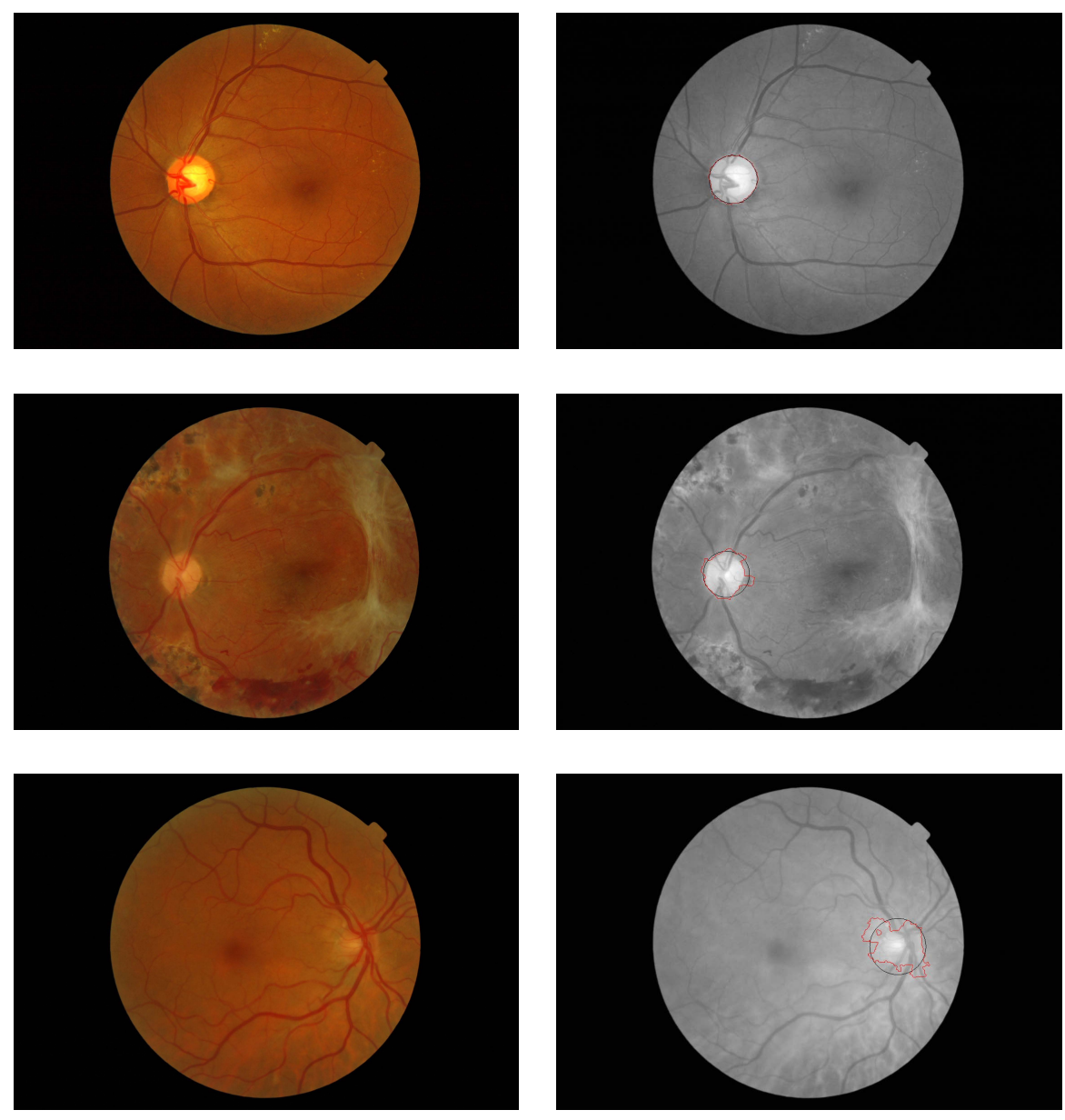

(a)

(b)

Figure 3.54: Some OD segmentation results on MESSIDOR database: (a) Original fundus images, (b) Results of the method proposed in Section 3.5 (OD contour in red and circular approximation of the OD contour in black). Top row: good segmentation. Middle row: fair segmentation. Bottom row: poor segmentation. 

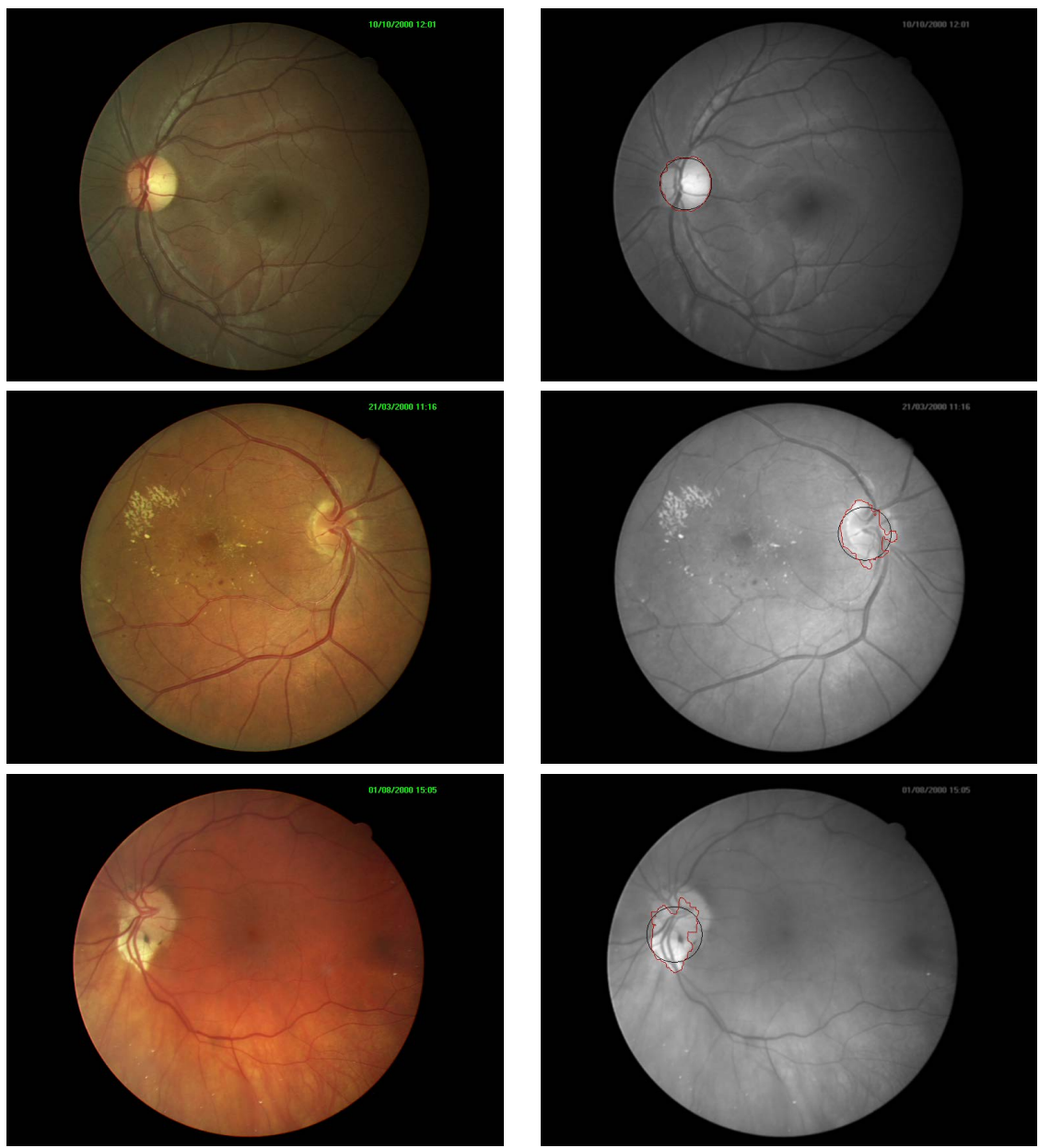

(a)

(b)

Figure 3.55: Some OD segmentation results on ONHSD database: (a) Original fundus images, (b) Results of the method proposed in Section 3.5 (OD contour in red and circular approximation of the OD contour in black). Top row: good segmentation. Middle row: fair segmentation. Bottom row: poor segmentation. 
As was mentioned in Section 3.1, most OD segmentation methods are divided into morphological algorithms, template-based methods, and deformable models. The proposed algorithm was compared with several methods of each category. Most works were selected due to the fact they use some of the analyzed databases, and thereby, the current and future comparison is facilitated.

- The presented method versus other morphological algorithms

On the one hand, Table 3.7 analyses further DRIONS database at the same time that the performance of our work is contrasted with the performance of other method based on mathematical morphology. So, first, the segmented images by the second observer were also compared with the gold standard to obtain inter-expert differences, and secondly, other existing technique based on marker-controlledwatershed transformation (Walter et al., 2002) was implemented and compared with ours.

Table 3.7: Comparison of the parameters of Table 3.4 achieved by the method proposed in Section 3.5, by the $2^{\text {nd }}$ observer and by other marker-controlledwatershed algorithm on DRIONS database.

\begin{tabular}{lccc}
\hline & JC & $\mathrm{S}$ & $\mathrm{Ac}$ \\
\hline Proposed method & $0.8424(0.1174)$ & $0.9084(0.0982)$ & $0.9934(0.0051)$ \\
$2^{\text {nd } \text { observer }}$ & $0.9202(0.0455)$ & $0.9578(0.0265)$ & $0.9970(0.0017)$ \\
Walter et al. (Wal- & $0.6227(0.3695)$ & $0.6813(0.3854)$ & $0.9689(0.0492)$ \\
ter et al., 2002) & & & \\
\hline \multicolumn{2}{l}{ TPF } & FPF & MAD \\
\hline Proposed method & $0.9281(0.1177)$ & $0.0040(0.0041)$ & $4.1098(3.4684)$ \\
$2^{\text {nd } \text { observer }}$ & $0.9498(0.0537)$ & $0.0012(0.0009)$ & $1.8887(1.1455)$ \\
Walter et al. (Wal- & $0.6715(0.3980)$ & $0.0210(0.0417)$ & $29.064(48.058)$ \\
ter et al., 2002) & & & \\
\hline
\end{tabular}


On the other hand, the concepts of Jaccard's coefficient (also known as area overlap) and the MAD included in Table 3.4 allow us to compare with the method proposed in (Welfer et al., 2010) as well as with other state-of-the-art algorithms that were analyzed in it. Table 3.8 compares the results of the method presented in Section 3.5 with the method that achieves the best results in (Welfer et al., 2010) on the same databases (DIARETDB1 and DRIVE).

Table 3.8: Comparison of the method proposed in Section 3.5 with the work presented in (Welfer et al., 2010) based on Jaccard's coefficient (JC) and mean absolute distance (MAD) on DIARETDB1 and DRIVE databases.

\begin{tabular}{lcc}
\hline & \multicolumn{2}{c}{ DIARETDB1 } \\
\hline Proposed method & $0.8173(0.1308)$ & $9.6759(8.4836)$ \\
Welfer et al. (Welfer et al., 2010) & $0.4365(0.1091)$ & $8.3100(4.0500)$ \\
\hline & \multicolumn{2}{c}{ DRIVE } \\
& JC & MAD \\
\hline Proposed method & $0.7163(0.1880)$ & $7.9981(9.9957)$ \\
Welfer et al. (Welfer et al., 2010) & $0.4147(0.0833)$ & 5.7400 \\
\hline
\end{tabular}

According to these data, the method of the Section 3.5 obtains a mean overlap greater than $70 \%$ for the five databases which significantly improves the results of the compared methods. As for MAD values, the results of Welfer et al. are slightly better. As the ground truth images of DIARETDB1 were resized from $1500 \times 1152$ pixels to $640 \times 480$, our results were also resized to have the same spatial resolution of ground truth and make the comparison possible.

- The presented method versus template-based methods

The validation on MESSIDOR database was performed through the comparison with a circular template-based method and four elliptical 
template-based approaches (Aquino et al., 2010). The methods analyzed in this work can be compared in based on Jaccard's coefficient. Three of the elliptical template-based variants were based on minimizing the algebraic distance (achieving a $J C \simeq 0.66$ ), and one was based on minimizing the geometric distance $(J C=0.67)$. Our method improves the performance of all elliptical approaches and achieves comparable results with the circular template-based method $(J C=0.86)$.

- The presented method versus deformable models

Our method was also tested on ONHSD database in order to compare with the tree approaches based on deformable models evaluated in (Lowell et al., 2004). In addition, with the aim of achieving a rigorous comparison, the same metric used in this work was calculated. A subjective perception of quality, which is based on four categories, was defined as a way of classifying the performance. For that, they defined the discrepancy as $\delta^{j}=\sum_{i} \frac{\left|m_{i}^{j}-\mu_{i}^{j}\right|}{\sigma_{i}^{j}+\epsilon}$, where $\mu_{i}^{j}$ and $\sigma_{i}^{j}$ summarize the clinician's choice of rim location on spoke $i$ of image $j$. Division by $\sigma$ compensates for uncertainty in rim position; $\epsilon=0.5$ is a small factor to prevent division by zero where the clinicians are in exact agreement. Each image is classified as Excellent, Good, Fair, or Poor depending on the discrepancy value (up to one, two, five, or more, respectively).

Table 3.9 summarizes the performance of our method on the subjective classification of the ONHSD dataset so that it is directly comparable with the results presented in (Lowell et al., 2004). While the best approach of this work has Excellent-Fair performance in 83\% of cases, our method obtains it in $94 \%$. 
Table 3.9: Subjective classification of performance on ONHSD dataset.

\begin{tabular}{lccccc}
\hline & Excellent & Good & Fair & Poor & Excellent-Fair \\
\hline Proposed method & $28 \%$ & $36 \%$ & $31 \%$ & $6 \%$ & $94 \%$ \\
$\begin{array}{l}\text { TemploralLock } \\
\begin{array}{l}\text { (Lowell et al., 2004) } \\
\text { Simple (Lowell }\end{array}\end{array}$ & $42 \%$ & $31 \%$ & $10 \%$ & $17 \%$ & $83 \%$ \\
$\begin{array}{l}\text { et al., 2004) } \\
\begin{array}{l}\text { DV-Hough (Lowell } \\
\text { et al., 2004) }\end{array}\end{array}$ & $39 \%$ & $22 \%$ & $20 \%$ & $19 \%$ & $81 \%$ \\
\hline
\end{tabular}

In addition to the comparison with other methods, different experiments were performed to demonstrate the robustness of the proposed algorithm:

1. The variability between fundus images in color, intensity, size, the presence of artefacts, etc. makes each state-of-the-art method uses a different input image: green (Lalonde et al., 2001; Eswaran et al., 2008; Niemeijer et al., 2009) and red (Walter et al., 2002; Hajer et al., 2008; Welfer et al., 2010) band of the original RGB image, or even a combination of both of them (Aquino et al., 2010; Lu, 2011), intensity component extracted from the HSI representation (Lowell et al., 2004) and lightness channel of the HLS space (Osareh et al., 2002). However, due to this fundus image variability, they do not always provided the desired results. Therefore a PCA, able to maximize the separation between the different objects of the image, was proposed as a more appropriate input image. For example, in Figure 3.56, PCA is compared with the use of the red component on a specific image. It can be observed that while the red component is completely oversaturated, PCA obtains a gray image where the OD could be segmented. Specifically, in Table 3.10 a quantitative analysis about the use of those two color planes can be observed concluding that the employment of PCA improves the final results. 


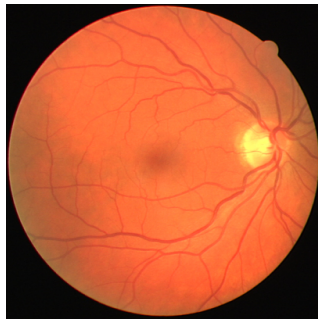

(a)

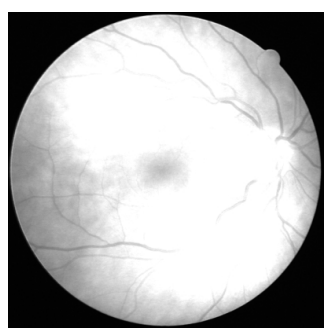

(b)

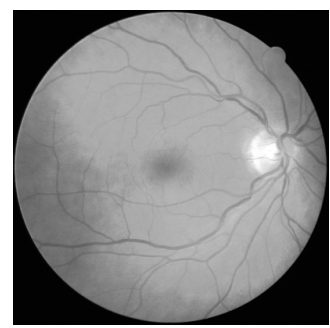

(c)

Figure 3.56: Advantages of PCA: (a) Original RGB fundus image, (b) Red component and (c) Image obtained by PCA.

Table 3.10: Comparison between the use of PCA versus the use of the red component on DRIVE database.

\begin{tabular}{lcc}
\hline & PCA & Red Component \\
\hline JC & $0.7163(0.1880)$ & $0.4106(0.3926)$ \\
$\mathrm{S}$ & $0.8169(0.1712)$ & $0.4674(0.4256)$ \\
$\mathrm{Ac}$ & $0.9903(0.0134)$ & $0.8979(0.2044)$ \\
$\mathrm{TPF}$ & $0.8544(0.1938$ & $0.5607(0.4368)$ \\
$\mathrm{FPF}$ & $0.0061(0.0085)$ & $0.0994(0.2343)$ \\
$\mathrm{MAD}$ & $7.9981(9.9957)$ & $72.741(87.216)$ \\
\hline
\end{tabular}

2. As for the improvements achieved by the proposed method in relation to others which use watershed transformation must also be highlighted. In particular, the different steps of our method help to avoid under-segmentation problems, as occurs in Figure 3.57 where the original image was segmented using only one internal marker located in the geodesic center of its largest and brightest object (Walter et al., 2002). It can be seen that there exist under-segmentation although the vessels were removed before applying the watershed transformation. Two different methods were used for vessel removal: a morphological closing and the inpainting technique presented in Subsection 3.5.1. 


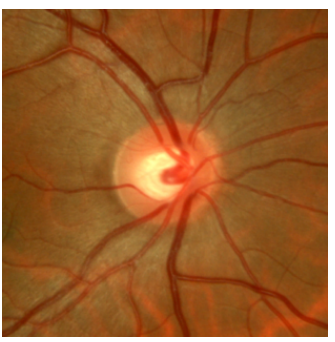

(a)

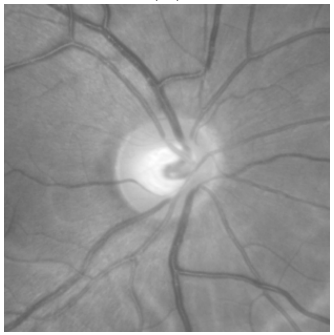

(d)

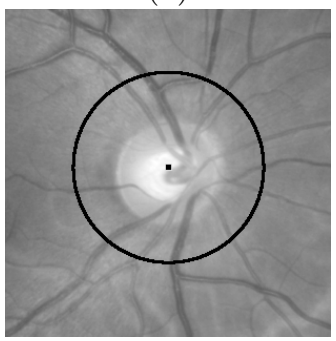

$(\mathrm{g})$

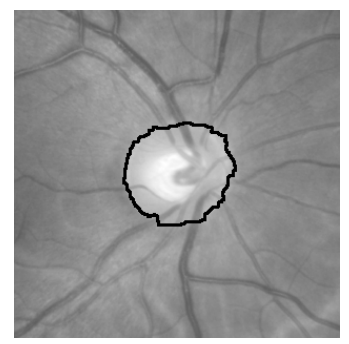

(b)

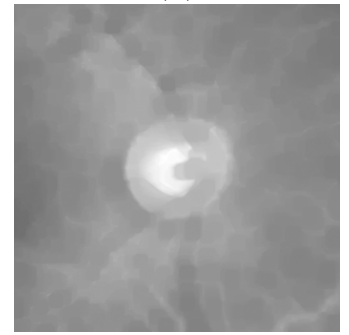

(e)

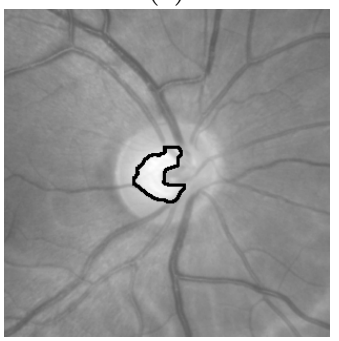

(h)

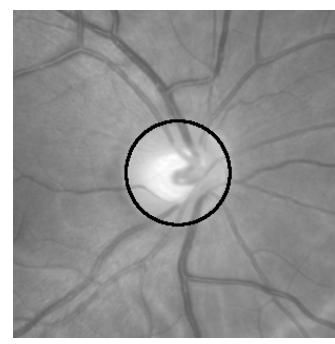

(c)

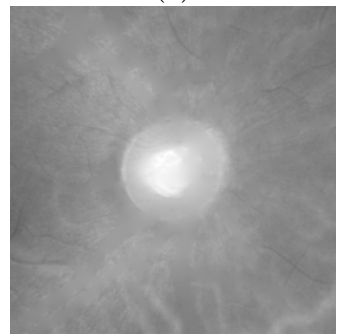

(f)

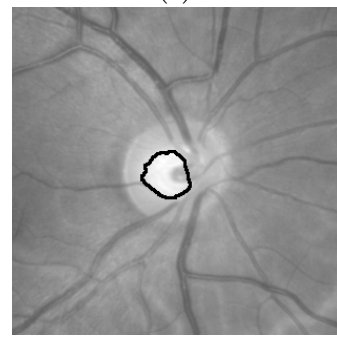

(i)

Figure 3.57: Under-segmentation problem produced in other markercontrolled-watershed method: (a) Original RGB image, (b) Watershed result of the method proposed in Section 3.5, (c) Circular approximation of (b), (d) Grayscale image, (e) Vessel removal through a morphological closing, (f) Vessel removal through inpainting technique, (g) Internal and external marker, $(h)$ Watershed region obtained on the gradient of (e) using the markers shown in $(g)$ and $(i)$ Watershed region obtained on the gradient of $(f)$ using the markers shown in $(g)$. 
3. Referring to the databases employed, most of the images included in them are pathological and therefore can distort the OD detection. For example, the images of patients with diabetic retinopathy may contain exudates which are of similar intensity to the OD or patients with glaucoma can suffer from peripapillary atrophy. Concretely, Figure 3.58 shows the robustness of the method in presence of exudates. However, if the lesion is as big as the optic disk and brighter, it is true that the method may fail.

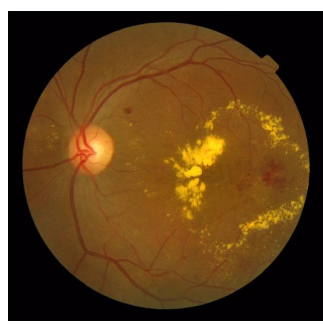

(a)

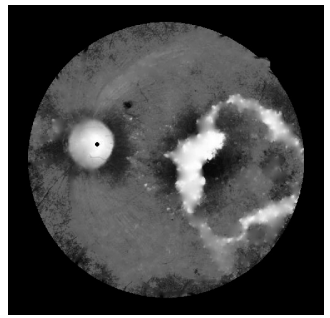

(b)

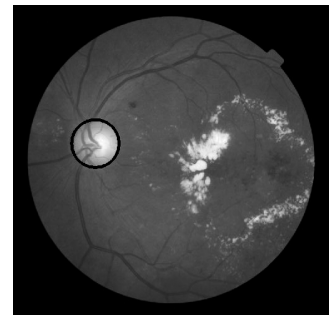

(c)

Figure 3.58: Robustness of the gray-image centroid in presence of exudates: (a) Original image, (b) First $P C\left(z_{1}\right)$ after inpainting technique with the centroid in black, and (d) Result of the proposed method.

\subsubsection{Computer-aided diagnosis software for hyperten- sive risk determination}

For the system validation presented in Section 3.6, a set of 67 fundus images was used. These images belong to a private database of the Fundación Oftalmológica del Mediterráneo (FOMDB). The implemented system was installed in this organization in order to be used by its clinicians in their daily practice and be able to carry out a clinic validation of the developed software based on a double-blind study. The fundus images were evaluated twice. Once, by qualified ophthalmologists who determined the presence or absence of vascular alteration through visual inspection of the original image, and another time using the developed system. A patient was considered hypertensive 
if the clinician detected in their fundus at least one sign of pathological arteriovenous crossing. The same three ophthalmologists rated the entire dataset with and without the software. A minimum period of one month was established between both rates to avoid the effect of the repeated exposure to the images. The final expert rates were obtained taking the majority vote among the three clinicians.

With the implemented tool, bifurcation angles, inside of an existing region of the original image concentric to the optic disk, were measured along with the caliber of a vein and an artery manually selected and situated at the same distance from the optic disk. Subsequently, a statistical analysis on different variables, extracted from data provided by the system, was conducted to see if any of them were able to discriminate whether a patient belonged to the group of hypertensive patients or to subjects without cardiovascular disease. Statistical Package for the Social Sciences (SPSS, IBM SPSS Data Collection) version 17.0 was used for this purpose.

Two parallel statistical studies were performed, one analyzing the variables related to the caliber and other analyzing those related to the bifurcation angles. The reason for the distinction was that only fundus images with at least five measured bifurcation angles were considered valid for this specific study. In both, the comparison between control and study groups was conducted using one-way ANOVA, where the dependent variables were each of the parameters calculated from the data provided by the software (vein caliber, artery caliber, relative caliber of the vein and artery (A/V ratio), average, median, deviation and variance of the branching angles, minimum and maximum angle and difference between the maximum and minimum angle) and the factor was the group formed by the subgroup without known disease and the subgroup of hypertensive patients.

Previous to the analysis, the normality of the calculated parameters was checked using the Kolmogorov-Smirnov test. From the obtained values, it can be concluded that only the caliber of the artery 
$(F(1 ; 66)=4.471 ; p<0.05)$ and the ratio between the caliber of the vein and the artery $(F(1 ; 66)=4.161 ; p<0.05)$ show statistically significant differences between both subgroups. Afterwards, using only these parameters, the optimal threshold to separate both classes was established and then the sensitivity, specificity and accuracy of the system to discriminate between healthy subjects and patients and with hypertensive pathology were calculated. Sensitivity and specificity measures the proportion of positives and negatives cases (pathological and healthy) which are correctly identified, respectively (Sens $=56.41 \%$ and Spec $=67.86 \%$ ). Accuracy is the proportion of true results (both true positives and true negatives) among the total number of cases examined $(A c=61.19 \%)$.

If the proportion of true results of the analyzed population is taken into account (i.e. the system accuracy), ophthalmologist visual diagnoses agreed with patient history $42 \%$ of the time without the computer assistance and that value increases up to $61 \%$ with the computer assistance. Their sensitivity and specificity are also improved from $38 \%$ and $50 \%$ to $56 \%$ and $68 \%$, respectively. Therefore, it can be concluded that the use of this computer-aided diagnosis software provides an improvement of almost $20 \%$ in hypertension detection.

Thanks to the software validation, it was demonstrated that the caliber of the arteries and the relative caliber of the veins and arteries show significant differences when patients are classified either healthy or hypertensive. Despite these results, it cannot be concluded that bifurcation angles are not significant for this purpose because only $54 \%$ of the fundus of the dataset could be analyzed due to the fact that the remainder contains less than 5 angles per image. Thus, the region where the measures are taken, should be enlarged or the database increased to repeat the same analysis.

Although the values of accuracy, sensitivity and specificity of the system are not too much high in the hypertension discrimination, it must be stressed that the results achieved by clinicians by visual 
inspection of the fundus are improved almost $20 \%$.

With regard to the obtained measures, they are accurate and reliable but also dependent on a correct skeleton detection and significant point classification.

\subsection{Conclusions}

In this chapter, several methods related to the detection and characterization of the retinal vessels and optic disk from a fundus image have been presented.

The method proposed in Subsection 3.3.1 for vessel segmentation is based mainly on morphological operators but also used principal curvature information, allowing an enhanced detection of the vessels on to the background of the image. This algorithm was compared with several methods and, apart from being among the best of the state of the art $(A c=0.9417)$, the optic disk edge is not detected as vessel, which facilitates a later vessel tracking process.

Subsection 3.3.2 deals with a method for vascular skeleton determination. This work proposes a new approach based on mathematical morphology and curvature evaluation and makes use of the stochastic watershed to extract the vessel centerline in a direct way. A correct vessel skeleton detection is usually required to analyze different vessel features that can indicate the presence of several diseases. Avoiding complete vessel segmentation supposes an improvement since the skeleton is not dependent of a previous stage at the same time that works properly in pathological images or with large changes in illumination and reduces over-segmentation problems of state-of-the-art methods based on a previous segmentation. With regard to other methods that obtain the skeleton in a direct way and use the marker-controlled watershed transformation, the stochastic watershed achieves a more robust detection and decreases the number of spurs. 
In Section 3.5, a new approach for the automatic detection of the optic disk was introduced. First, it is focused on the use of a new gray image as input obtained through PCA which combines the most significant information of the three RGB components. Secondly, several operations based on mathematical morphology are implemented with the aim of locating the OD. For that purpose, both stochastic and stratified watershed as well as geodesic transformations, such as gray level distance and gray level close holes, were used. The algorithm was validated on 5 different public databases obtaining promising results and improving the results of other methods of the literature $(J C=0.8200$, $S=0.8932, A c=0.9947, T P F=0.9275, F P F=0.0036$, and $M A D=5.9060)$. These values correspond with the weighted average of the results of the proposed method, i.e., they are calculated according to the number of images of each analyzed database.

Making use of the aforementioned methods, a computer-aided diagnosis software for hypertensive risk determination was presented in Section 3.6. From a fundus image, the tool automatically detects blood vessels and allows measurement of bifurcation angles and selection of branches to determine their caliber. These data facilitate expert medical diagnosis and study of the progression of the disease. In particular, the software was used by expert ophthalmologists to help them to discriminate between a normal vascularization and cardiovascular pathology. The accuracy, sensitivity and specificity of the hypertension detection achieved by direct visual inspection of the fundus were improved by almost $20 \%$. 


\section{Chapter 4}

\section{Retina background characterization}

This chapter focuses on analyzing the texture of the fundus images by means of local binary patterns to differentiate between pathological and healthy images. In particular, its goal is to distinguish between diabetic retinopathy (DR), age-related macular degeneration (AMD) and normal fundus images analyzing directly the retina background texture and avoiding a previous lesion segmentation stage. Chapter 4 arose from a research stay in the University of Stavanger under the supervision of Prof. Kjersti Engan.

The information contained in this chapter was included in a journal paper that is currently under revision:

Morales, S., Engan, K., Naranjo, V., Colomer, A. (2015). Retinal Disease Screening through Local Binary Patterns. IEEE Journal of Biomedical and Health Informatics. 
Contents

4.1 Introduction . . . . . . . . . . . . . 119

4.2 Theoretical background . . . . . . . . . . 121

4.3 Application to pathology detection: Diabetic retinopathy and age-related macular degeneration .............. 124

4.3 .1 General description . . . . . . . . . . . . . . 124

4.3.2 Feature extraction . . . . . . . . . . . . . . . 124

4.3 .3 Classification . . . . . . . . . . . . . . . 125

4.4 Results . . . . . . . . . . . . . 130

4.4 .1 Material . . . . . . . . . . . . . . . . 130

4.4 .2 Experiments . . . . . . . . . . . . . . 134

4.5 Conclusions . . . . . . . . . . . . . 139 


\subsection{Introduction}

Diabetic retinopathy (DR) and age-related macular degeneration (AMD) are nowadays two of the most frequent causes of blindness and vision loss (World Health Organization, 2010a). In addition, these diseases will experience a high growth in the future due to diabetes incidence increase and aging population in the current society. Based on these facts, a computer-aided diagnosis software capable of discriminating, through image processing, between a healthy fundus (without any pathology) and DR and AMD patients is presented in this chapter. Specifically, the aim of the proposed software is to carry out an automatic screening of these diseases facilitating the at-risk population assessment.

DR and AMD can be characterized by the presence of specific types of retinal lesions such as microaneurysms, exudates or drusen, among others, as was seen in Section 2.2. Figure 4.1 depicts some examples of these diseases in comparison with the fundus image from a healthy subject.

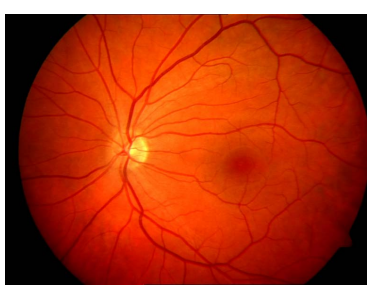

(a)

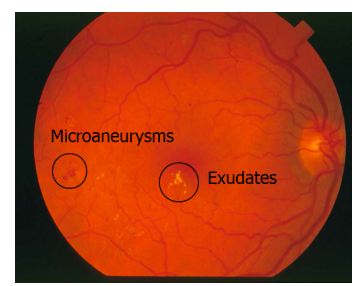

(b)

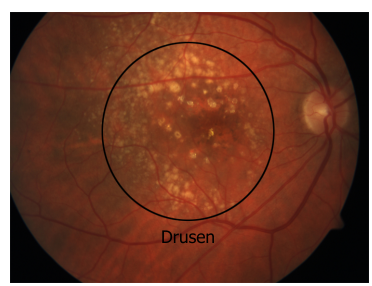

(c)

Figure 4.1: Fundus images. (a) Healthy, (b) DR (with microaneurysms and exudates) and (c) AMD (with drusen).

This chapter focuses on investigating the discrimination capabilities in the fundus texture to differentiate between pathological and healthy images. In particular, the main focus lies in exploring the performance of Local Binary Patterns (LBP) as a texture descriptor for retinal 
images. LBP is a technique used for describing textures that has been given a lot of attention in recent years (Ojala et al., 2001; Ojala et al., 2002). It is based on looking at the local variations around each pixel, and assigning labels to different local patterns. Thereafter, the distribution of the labels is evaluated and used in the classification stage. There are many examples of the success of LBP used to describe and classify textures in general (Ahonen et al., 2006; Heikkil 2009; Liao et al., 2009; Yang and Ai, 2007) and also in the case of medical imaging (Kotu et al., 2012; Oppedal et al., 2012; Nanni et al., 2010). However, regarding fundus image processing, LBP has not been widely used. Most state-of-the-art works that use the LBP technique on fundus images focus on the segmentation of the retinal vessels (Zabihi et al., 2010; Dhanushkodi and Vasuki, 2013) rather than on a full diagnosis system, although some examples can be found in this direction (Garnier et al., 2014; Mookiah et al., 2013). Garnier et al. deal with the AMD detection using LBP. The texture information on several scales is analyzed through wavelet decomposition and the LBP histogram is found from the wavelet coefficients. Linear Discriminant Analysis (LDA) is used for feature dimension reduction using the values of the entire LBP histogram as input features. Image classification on a set of 45 images is evaluated with a leave-one-out validation method (Garnier et al., 2014). Mookiah et al. extract abnormal signs from fundus images to detect normal fundus and two DR stages. Thirteen features, such as the area of hard exudates, the area of blood vessels, bifurcation points, textures and entropies, fed three different classifiers (Probabilistic Neural Network (PNN), Decision Tree C4.5 and Support Vector Machine (SVM)). The textures are found by LBP and Laws energy. A previous segmentation of the exudates, optic disk and blood vessels is needed for feature extraction. The experiments were conducted on 156 subjects and the PNN was chosen as the best classifier with three-fold cross validation (Mookiah et al., 2013).

The goal of this chapter is to distinguish between DR, AMD and normal fundus images at the same time and avoiding any previous segmentation stage of retinal lesions. The texture of the retina 
background is directly analyzed by means of LBP, and only this information is used to differentiate healthy patients and these two pathologies. A comprehensive study about what type of classifier obtains the best results is also undertaken. The performance of Logistic Regression, Neural Networks, SVM, Naive Bayes, J48, Rotation Forest, Random Forest and AdaBoost M1 is compared. This approach is different from previous works. Mookiah et al. require the segmentation of exudates in addition to segmentation of main structures (optic disk and vessels) for feature extraction and, although three different classes are identified, they only focus on DR detection. Garnier et al. do not need previous segmentations but only handle with AMD diagnosis. Both works use directly the information of the LBP histograms. However, in this work, LBP values are combined with a contrast measure and, instead of using directly the histograms values, different statistics are calculated from them.

The rest of the chapter is organized as follows: in Section 4.2 theoretical background is included and in Section 4.3 the proposed method is presented. Section 4.4 shows how system validation was performed and as well as the obtained results. Finally, Section 4.5 provides conclusions and some future areas for work.

\subsection{Theoretical background}

As Section 3.2, this section is intended as a guide to the reader about the main tool used in the chapter: local binary pattern theory.

Local binary pattern (LBP) is a powerful gray-scale texture operator used in many computer vision applications because of its computation simplicity (Ojala et al., 2001; Ojala et al., 2002). The first step in LBP is to produce a label for each pixel in the image where the label is found based on the local neighborhood of the pixel which is defined by a radius, $R$, and a number of points, $P$. The neighboring pixels are thresholded with respect to the gray value of the central pixel of the neighborhood generating a binary string or, in other words, a binary pattern. The 
value of a LBP label is obtained for every pixel by summing the binary string weighted with powers of two. It can be represented as follows:

$$
L B P_{P, R}=\sum_{p=0}^{P-1} s\left(g_{p}-g_{c}\right) \cdot 2^{p}, \quad s(x)= \begin{cases}1 & \text { if } x \geq 0 \\ 0 & \text { if } x<0\end{cases}
$$

where $g_{p}$ and $g_{c}$ are the gray values of the neighborhood and central pixel, respectively. $P$ represents the number of samples on the symmetric circular neighborhood of radius $R$. The $g_{p}$ values are interpolated to fit with a given $R$ and $P$. The values of the labels depend on the size of the neighborhood $(P)$. In Figure 4.2 two neighborhoods with different radii, but the same number of samples, are drawn.

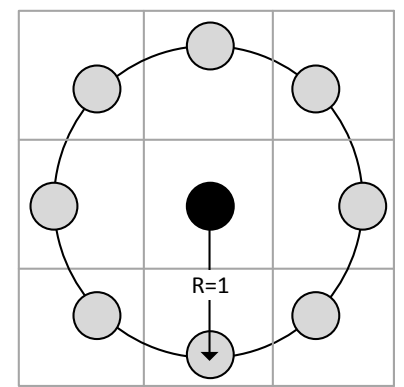

(a)

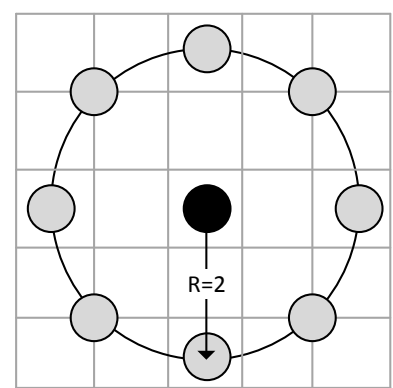

(b)

Figure 4.2: LBP neighborhoods: (a) $R=1$ and $P=8$. (b) $R=2$ and $P=8$.

$2^{P}$ different binary patterns can be generated in each neighborhood. However, the bits of these patterns must be rotated to the minimum value to achieve a rotation invariant pattern. For example, the binary patterns 01110000, 10000011 and 00111000 are different rotations of the same pattern and correspond to the normalized string 00000111. In the case of $P=8$, only 36 of the $2^{P}$ possible patterns are rotation invariant, i.e., $L B P_{8, R}$ can have 36 different values. Figure 4.3 shows 
how LBP is calculated for a circular neighborhood of radius $1(R=1)$ and 8 samples $(P=8)$.

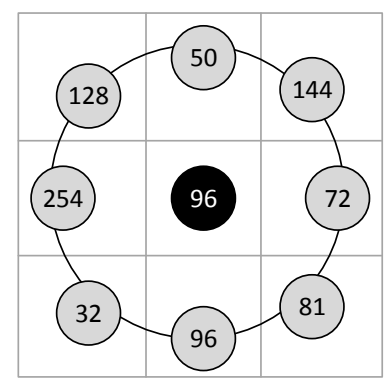

(a)

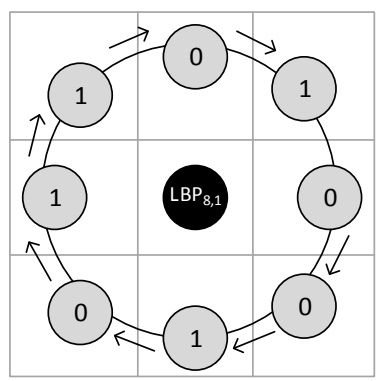

(b)

Figure 4.3: LBP computation: (a) Gray values of a circular neighborhood of radius 1 and 8 samples. (b) Thresholding between the gray value of the neighborhood and the central pixel. The rotation invariant local binary pattern generated is 00101101 (the arrows indicate the order in which the string is formed). Specifically, the LBP label is obtained as follows $L B P_{8,1}=$ $0 \times 2^{0}+0 \times 2^{1}+1 \times 2^{2}+0 \times 2^{3}+1 \times 2^{4}+1 \times 2^{5}+0 \times 2^{6}+1 \times 2^{7}=180$.

When LBP is used for texture description, it is common to include a contrast measure by defining the rotational invariant local variance as follows:

$$
V A R_{P, R}=\frac{1}{P} \sum_{p=0}^{P-1}\left(g_{p}-\mu\right)^{2}, \quad \mu=\frac{1}{P} \sum_{p=0}^{P-1} g_{p} .
$$

The LBP and VAR measures are complementary and are combined to enhance the performance of the LBP operator. The implementation of both measures is publicly available online. ${ }^{1}$

\footnotetext{
${ }^{1}$ www.ee.oulu.fi/ gyzhao/LBP_Book.htm
} 


\subsection{Application to pathology detection: Dia- betic retinopathy and age-related macular degeneration}

Using the technique explained above, an algorithm able to distinguish between healthy retinal images and images belonging to patients with diabetic retinopathy and age-related macular degeneration was developed.

\subsubsection{General description}

The algorithm proposed for classification of retina images does not need a prior segmentation of suspicious lesions. Manual segmentation of lesions are time consuming and automatic segmentation algorithms might not be accurate, thus removing the need for lesion segmentation can make the classification more robust. The algorithm is based on the study of the retina background. Hence, the main structures of the fundus (the vascular network and the optic disk), which are not related to the diseases under study, should not be taken into account when the texture of the fundus is analyzed. If these predominant structures were included in the image texture analysis, they could mask the difference between healthy and pathological images because their aspect in both cases would be similar.

\subsubsection{Feature extraction}

The LBP and VAR operators described above are used to characterize the texture of the retina background. They are calculated for each pixel of the RGB images using $P=8$ and different values of $\mathrm{R}$ $(R=\{1,2,3,5\})$. Due to the fact that the images under study belong to different databases, the size of the images varies. As the LBP and VAR depend on the radius of the neighborhood, the images must be resized to a standardized size to obtain comparable texture descriptors. The images are resized using the length of the horizontal diameter of 
4.3. Application to pathology detection: Diabetic retinopathy and age-related macular degeneration

the fundus as reference (Zhang et al., 2012). Bicubic interpolation is used for resizing; the output pixel value is a weighted average of pixels in the nearest 4-by-4 neighborhood.

The red, green and blue components of each image are independently analyzed. One example of the aspect of the LBP and VAR images of an AMD fundus is represented in Figure 4.4. Only the pixels of the retina background are considered significant for the texture analysis. Thus the LBP and VAR values corresponding to pixel positions of the vessels, the optic disk or outside the fundus should not be taken into account. The vascular network and the optic disk are detected by the methods presented in Subsection 3.3.1 and Section 3.5 and, subsequently, the LBP and VAR labels are removed according to the pixel position of the optic disk and the vascular network. The external mask is directly obtained by thresholding.

The resulting LBP and VAR images provide a description of the image texture. After masking the optic disk and vessel segments, the LBP and VAR values within the external mask of the fundus are collected into histograms, one for each color (RGB). Different statistical information is extracted from these histograms to use it as features in the classification stage. Concretely, the calculated statistical values are: mean, standard deviation, median, entropy, skewness and kurtosis. To sum up, 6 statistical values are calculated from each LBP and VAR histogram, giving place to 12 features for each radius used. Consequently, the total number of features is equal to 144 (12 features $\mathrm{x} 4$ radius $\mathrm{x} 3$ components). Figure 4.5 depicts the feature extraction flowchart and Algorithm 4.1 summarizes the steps to be followed.

\subsubsection{Classification}

The dataset analyzed was divided into two subsets, one for training and testing by cross validation (model set) and other purely for testing (validation set). 


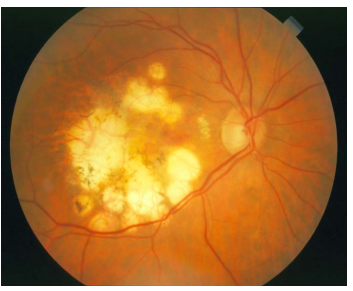

(a)

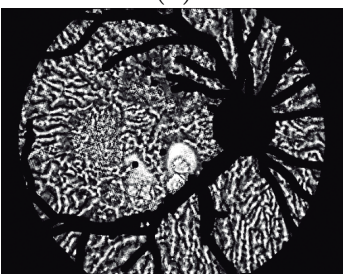

(d)

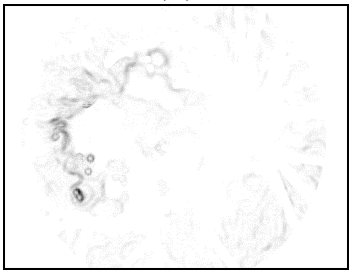

(g)

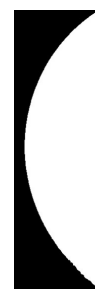

(b)

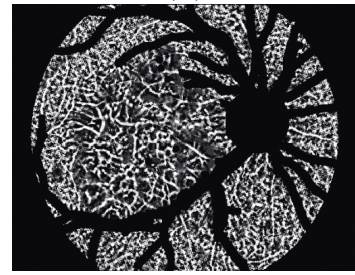

(e)

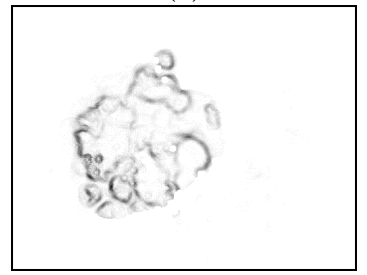

(h)

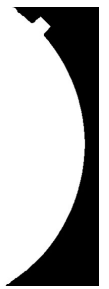

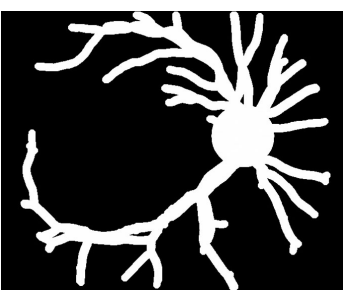

(c)

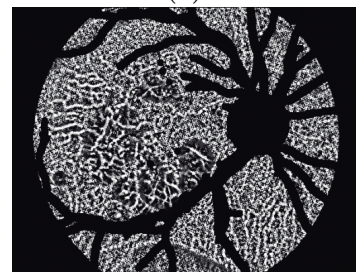

(f)

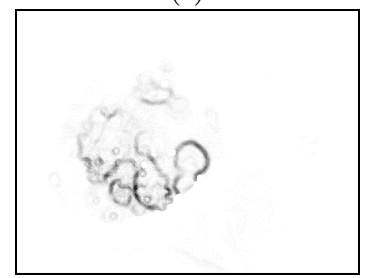

(i)

Figure 4.4: Feature extraction using $P=8$ and $R=5$. (a) AMD fundus image, (b) External mask, (c) Optic disk and vessel mask, (d-f) LBP images calculated on $R, G$ and $B$ components, respectively. Optic disk and vessel segments are removed (black). (g-i) VAR images calculated on $R, G$ and $B$ components, respectively. Optic disk and vessel segments are removed (white).

Once the features are extracted, the data of the model set must be preprocessed before the classification stage. In the preprocessing, two tasks are carried out: data normalization and data resampling. The first one because the range of values of raw data varies widely and the second one because the dataset is clearly unbalanced and most machine learning algorithms would not work properly. In particular, the method used for the normalization is to standardize all numeric attributes in the given 


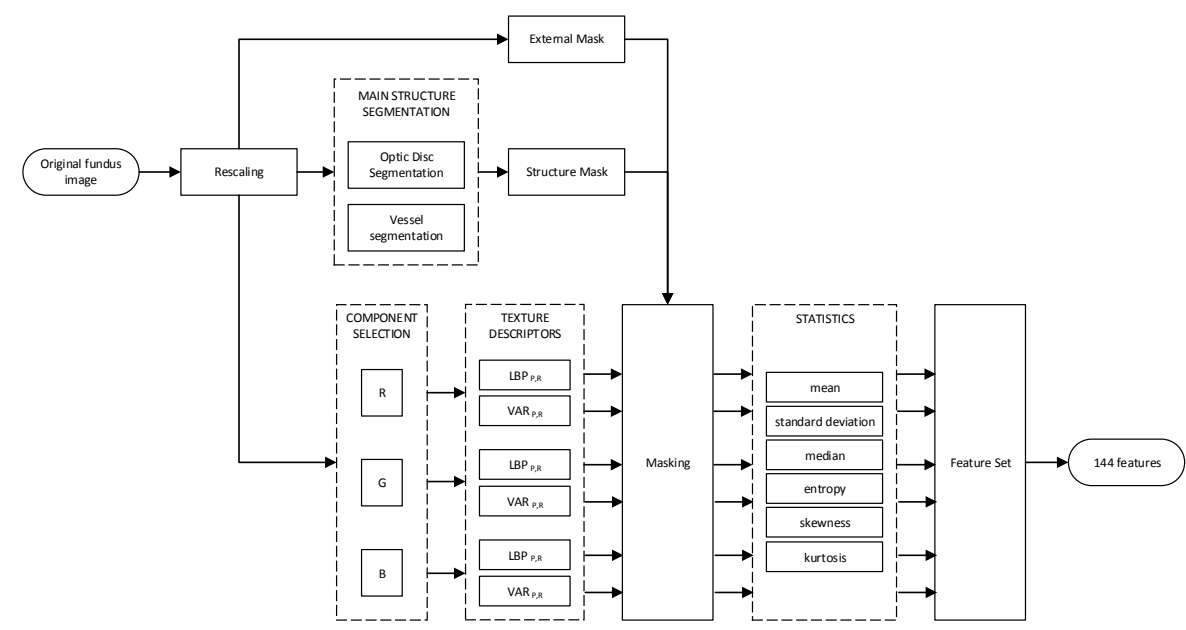

Figure 4.5: Feature extraction flowchart. First, the original fundus image is rescaled based on its horizontal diameter. Secondly, LBP and VAR measures are performed on the three $R G B$ components using $P=8$ and $R=\{1,2,3,5\}$. Then, the external mask of the fundus is determined and the optic disk and vessels are segmented generating a structure mask. Both, external and structure masks, are used for masking the result of the texture descriptors. The final feature set is formed by the statistical values of all texture descriptors after masking.

dataset to have zero mean and unit variance and, for the resampling, the Synthetic Minority Oversampling TEchnique (SMOTE) (Chawla et al., 2002) is applied. This technique over-samples the minority class creating synthetic minority class examples to balance the dataset.

Afterwards, external cross validation (CV), also called nested CV (Scheffer, 1999; Dudoit and van der Laan, 2005), is performed on the model set so that the dimensionality of the data is reduced by feature selection before being passed on to a classifier. 10 folds are used in the external loop and 5 folds in the internal loop. The purpose of the internal loop is to select a feature subset and the used technique is 


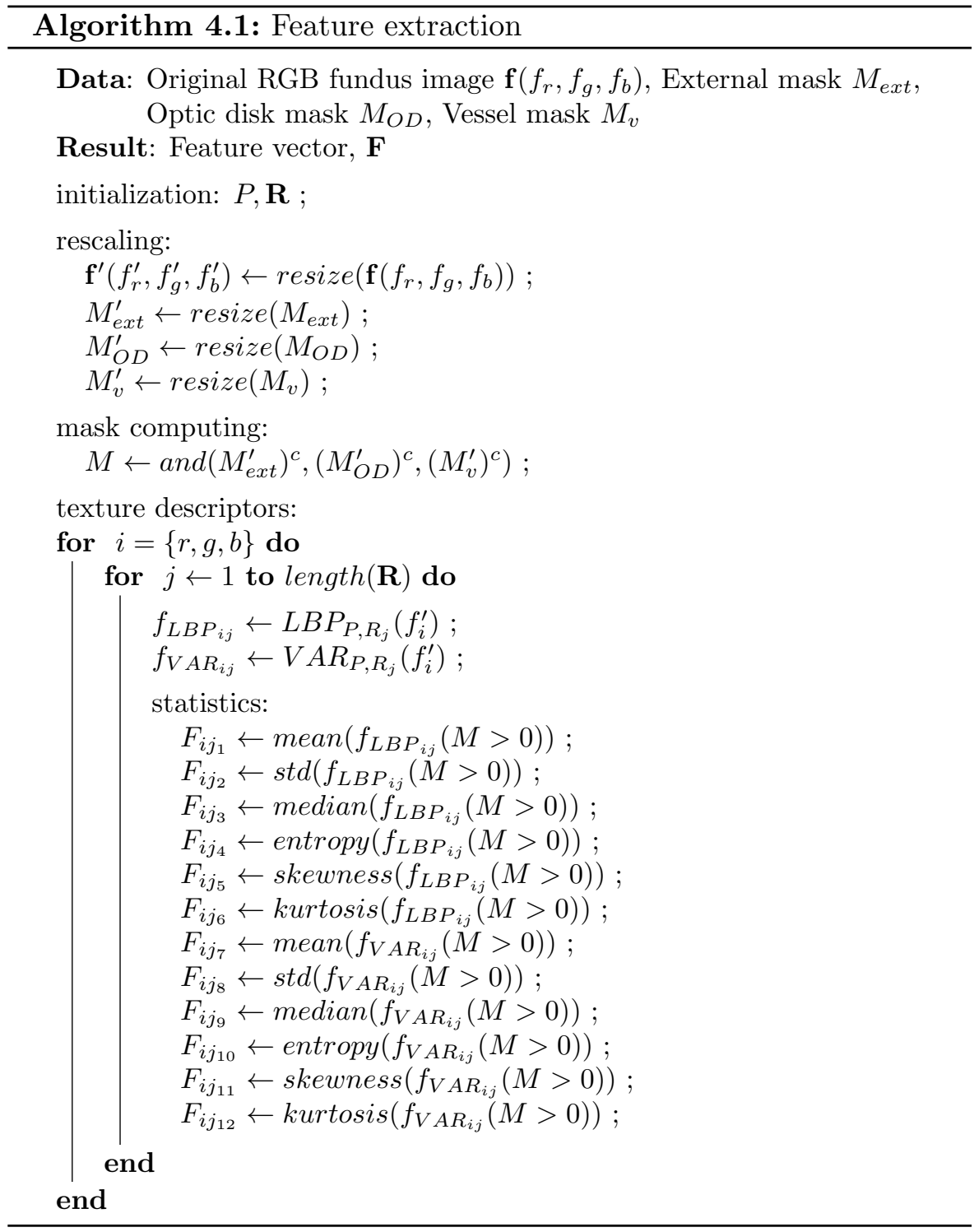

a wrapper method (Kohavi and John, 1997) with forward (best first) 
4.3. Application to pathology detection: Diabetic retinopathy and age-related macular degeneration

selection. The same type of classifier is used in both the internal and external loops. The external loop divides the set into 10 non-overlapping pairs of training (90\%) and test (10\%) sets. For each fold of the external $\mathrm{CV}$, the training set is further divided into 5 non-overlapping sets by the internal CV loop. The internal loop is done first to select the feature subset of this particular fold of the external loop. Thereafter, the external loop trains the classifier using this subset, and tests it on the remaining $10 \%$. This is repeated for every fold. Notice that the feature set might vary with each external fold of the CV scheme. Thus doing an external or nested CV gives a measure of how well the method works for this dataset, where the method includes the feature subset selector and the choice of classifier. Figure 4.6 shows how the external $\mathrm{CV}$ is performed for the first fold.

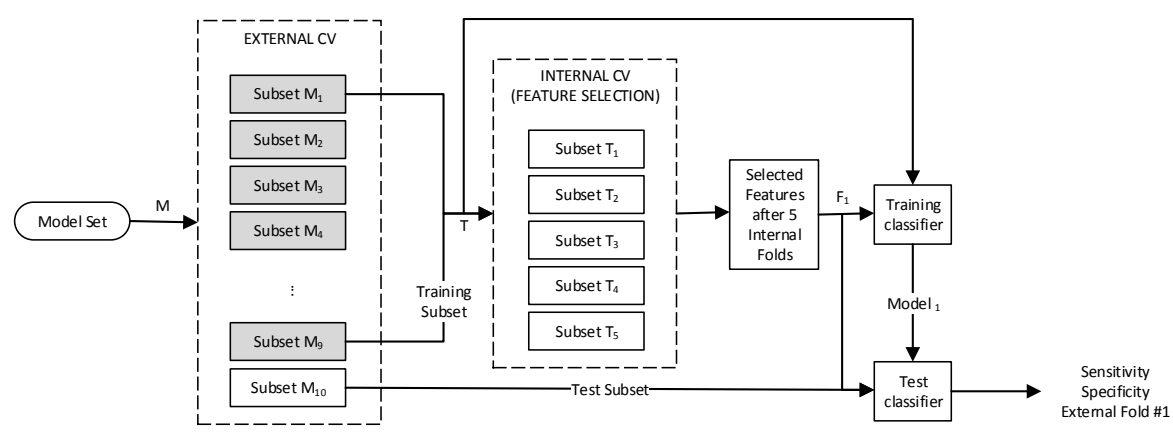

Figure 4.6: External cross validation flowchart. The process is repeated iteratively for the 10 folds of the external loop.

Finally, a final classifier is made using the whole model set for feature subset selection and thereafter the whole model set is used for training the classifier. The validation set is tested on the final classifier. The process is summarized in Figure 4.7. The normalization parameters from the model set are saved as a part of the classifier, such that the validation set is normalized using these same parameters.

Both, data preprocessing and classification, were carried out with 


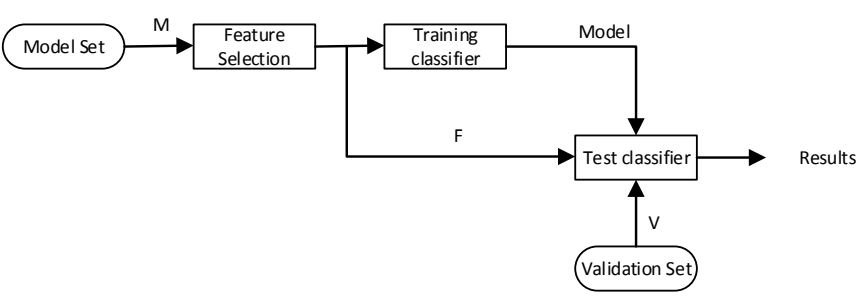

Figure 4.7: Final validation flowchart.

Weka (Hall et al., 2009; Witten et al., 2011).

\subsection{Results}

This section describes the material used and the experiments designed for method validation.

\subsubsection{Material}

The material used in this work are images previously diagnosed as normal (without known pathology), DR or AMD. The dataset was composed of images from 4 different databases which included some of the categories under study:

\section{ARIA}

ARIA database (Farnell et al., 2008; Zheng et al., 2012) is formed by 143 color fundus images $(768 \times 576$ pixels $)$, which are organized into three classes: age-related macular degeneration (AMD) subjects $(n=23)$, healthy control-group subjects $(n=61)$, and diabetic subjects $(\mathrm{n}=59) .^{2}$ Trained image analysis experts traced out the blood vessels, and also the optic disk and fovea where relevant.

\footnotetext{
$2_{\text {ww. }}$ eyecharity.com/aria_online.html
} 


\section{STARE}

STARE database (Hoover et al., 2000; Hoover and Goldbaum, 2003) is a full set of 402 images $(700 \times 605$ pixels $)$ where thirteen different diagnoses were considered. ${ }^{3}$ From this dataset, three subsets were generated: age-related macular degeneration $(n=47)$, normal $(n=37)$, and diabetic retinopathy $(\mathrm{n}=89)$.

\section{Е-ОРНТНА}

E-OPHTHA (Decencière et al., 2013) is a database of fundus images especially designed for diabetic retinopathy screening. ${ }^{4}$ It contains 257 images with no lesion, 47 images with exudates and 148 with microaneurysms or small hemorrhages making a total of 174 images with diabetic retinopathy.

\section{DIAGNOS}

DIAGNOS (Garnier et al., 2014) is a private database, property of DIAGNOS Inc., composed of 45 fundus images, 22 afflicted with AMD and 23 healthy.

Both E-OPHTHA and DIAGNOS have a range of different image resolutions. The four databases experience a significant variability in color, illumination, resolution, quality, etc. both within and, even more, among the databases. Some representative images of each database are shown in Figure 4.8.

All images of the resulting dataset must comply with certain quality criteria. The following causes were considered reasons for exclusion:

- Images with artefacts, for example bright and circular spots produced by some dust in the camera lens.

\footnotetext{
${ }^{3}$ www.ces.clemson.edu/ ahoover/stare/

${ }^{4}$ www.adcis.net/en/Download-Third-Party/E-Ophtha.html
} 


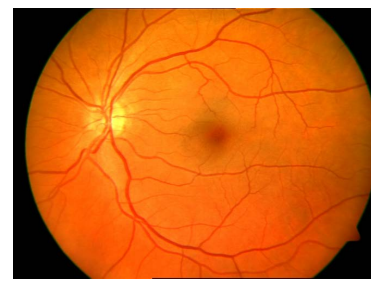

(a)

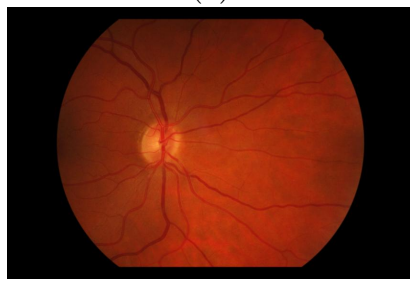

(c)

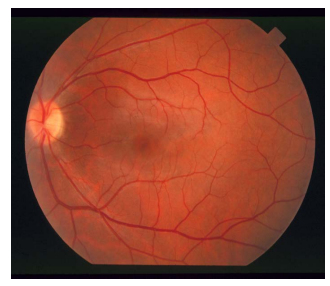

(b)

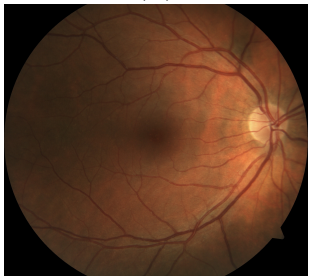

(d)

Figure 4.8: Fundus images belonging to the different used databases. (a) ARIA, (b) STARE, (c) E-OPHTHA and (d) DIAGNOS.

- Images affected by a relative large amount of impulsive noise (salt and pepper noise).

- Images where the vascular network is largely over-segmented by the method presented in Subsection 3.3.1.

- Images with a doubtful diagnosis. ${ }^{5}$

- Images with highlights around the vessels associated with young retinas. 6

- Tessellated images due to the fact there are lesser amounts of pigment in the retinal pigment epithelium. ${ }^{6}$

\footnotetext{
${ }^{5}$ Based on second opinion from a medical doctor.

${ }^{6}$ This situation dramatically changes the images, thus they are not included here. However they should be regarded separately in future studies since it is highly desirable to be able to include these types of subjects.
} 
Figure 4.9 depicts some of these cases. Most of these choices were done to determine if LBPs were able to discriminate between healthy and pathological images in a normal situation or, in other words, without the presence of distracting elements. When this hypothesis was confirmed, particular cases will be included in the analysis.

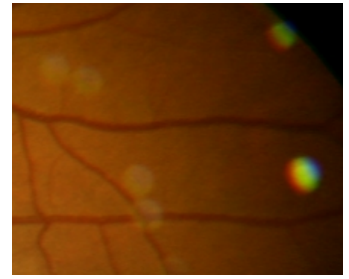

(a)

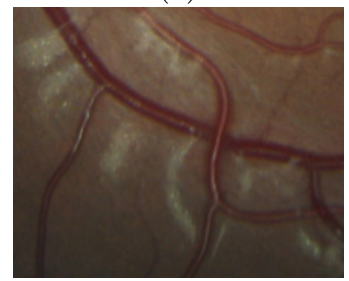

(c)

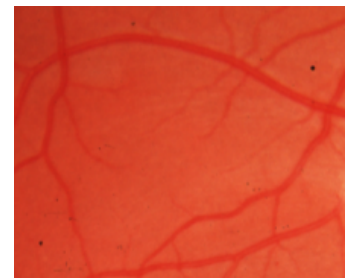

(b)

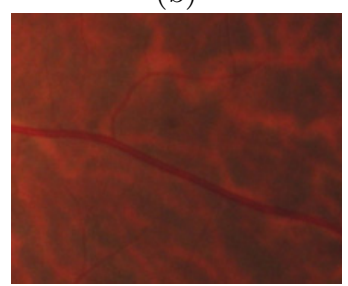

(d)

Figure 4.9: Excluded images. (a) With artefacts, (b) Noisy, (c) With highlights and (d) Tessellated.

After exclusion, the resulting dataset used in this work is formed by a total of 251 images. In Table 4.1, the number of images of each database after the exclusion criteria is detailed. As mentioned before, this dataset was divided into two subsets, one for training and testing by cross validation (model set) and other, completely independent, purely for testing (validation set). The model set contains $80 \%$ of the images and the validation set the remaining $20 \%$. Table 4.2 shows the contents of each subset. 
Table 4.1: Content of each database.

\begin{tabular}{lccccc}
\hline & ARIA & STARE & E-OPHTHA & DIAGNOS & Total \\
\hline AMD & 9 & 23 & 0 & 17 & 49 \\
DR & 8 & 35 & 37 & 0 & 80 \\
NORMAL & 30 & 13 & 79 & 0 & 122 \\
\hline Total & 47 & 71 & 116 & 17 & 251 \\
\hline
\end{tabular}

Table 4.2: Content of the model and validation sets.

\begin{tabular}{lccccc}
\hline & & \multicolumn{5}{c}{ Model set } \\
& ARIA & STARE & E-OPHTHA & DIAGNOS & Total \\
\hline AMD & 7 & 18 & 0 & 14 & 39 \\
DR & 6 & 28 & 29 & 0 & 63 \\
NORMAL & 24 & 10 & 63 & 0 & 97 \\
\hline Total & 37 & 56 & 92 & 14 & 199 \\
\hline & ARIA & STARE & E-OPHTHA & DIAGNOS & Total \\
& 2 & 5 & 0 & 3 & 10 \\
AMD & 2 & 7 & 8 & 0 & 17 \\
DR & 6 & 3 & 16 & 0 & 25 \\
NORMAL & 10 & 15 & 24 & 3 & 52 \\
\hline Total & & & & & \\
\hline & & & & &
\end{tabular}

\subsubsection{Experiments}

Five experiments were conducted and validated with the procedure proposed in this chapter: 
- AMD - Normal

- DR - Normal

- Pathological (including AMD and DR) - Normal

- AMD - DR

- 3 class problem (AMD - DR - Normal)

For each experiment, different classifiers were tested: Logistic Regression (Cessie and van Houwelingen, 1992), Neural Networks (Hecht-Nielsen, 1990), SVM (Chang and Lin, 2011), Naive Bayes (John and Langley, 1995), C4.5 (Quinlan, 1993), Rotation Forest (Rodriguez et al., 2006), Random Forest (Breiman, 2001), and AdaBoost (Freund and Schapire, 1996). The performance of the algorithms was evaluated based on two concepts: sensitivity or true positive rate (TPR) and specificity or true negative rate (TNR). Sensitivity and specificity measure the proportion of positive and negative cases which are correctly identified as such, respectively. Tables 4.3 and 4.4 detail the sensitivity and specificity obtained on the model and validation sets in all the experiments with the different classifiers. In the case of the three-class problem, the average sensitivity and specificity are shown. In Table 4.3, the best results of each experiment are highlighted in bold. The metrics computed after external cross validation are additive over the folds, i.e. the correctly or incorrectly classified cases are summed over the folds and divided by the total number of instances. 
Table 4.3: Results of the different experiments on the model set (external $C V)$.

\begin{tabular}{|c|c|c|c|c|c|c|}
\hline & \multicolumn{2}{|c|}{ AMD-Normal } & \multicolumn{2}{|c|}{ DR-Normal } & \multicolumn{2}{|c|}{ Path.-Normal } \\
\hline & TPR & TNR & TPR & TNR & TPR & TNR \\
\hline Logistic Regression & 0.948 & 0.990 & 0.732 & 0.814 & 0.843 & 0.861 \\
\hline Neural Networks & 0.866 & 0.979 & 0.722 & 0.773 & 0.833 & 0.832 \\
\hline SVM & 0.938 & 0.959 & 0.773 & 0.876 & 0.784 & 0.842 \\
\hline Naive Bayes & 0.990 & 0.959 & 0.588 & 0.794 & 0.647 & 0.782 \\
\hline C4.5 & 0.969 & 0.938 & 0.897 & 0.804 & 0.853 & 0.851 \\
\hline Rotation Forest & 0.938 & 0.959 & 0.835 & 0.866 & 0.892 & 0.881 \\
\hline Random Forest & 0.959 & 0.959 & 0.876 & 0.866 & 0.843 & 0.861 \\
\hline \multirow[t]{3}{*}{ AdaBoost } & 0.979 & 0.959 & 0.897 & 0.814 & 0.824 & 0.772 \\
\hline & & \multicolumn{2}{|c|}{$A M D-D R$} & \multicolumn{2}{|c|}{$A M D-D R$-Normal } & \\
\hline & & $\mathrm{TPR}$ & TNR & TPR & TNR & \\
\hline \multicolumn{2}{|c|}{ Logistic Regression } & 0.873 & 0.937 & 0.835 & 0.918 & \\
\hline \multicolumn{2}{|c|}{ Neural Networks } & 0.905 & 0.921 & 0.842 & 0.921 & \\
\hline \multicolumn{2}{|l|}{ SVM } & 0.841 & 0.968 & 0.852 & 0.926 & \\
\hline \multicolumn{2}{|l|}{ Naive Bayes } & 0.857 & 0.952 & 0.777 & 0.888 & \\
\hline \multicolumn{2}{|l|}{$\mathrm{C} 4.5$} & 0.889 & 0.937 & 0.849 & 0.924 & \\
\hline \multicolumn{2}{|c|}{ Rotation Forest } & 0.921 & 0.937 & 0.883 & 0.942 & \\
\hline \multicolumn{2}{|c|}{ Random Forest } & 0.921 & 0.921 & 0.907 & 0.954 & \\
\hline \multicolumn{2}{|l|}{ AdaBoost } & 0.921 & 0.968 & 0.808 & 0.904 & \\
\hline
\end{tabular}

With regard to the obtained results after external CV (Table 4.3), the experiment with the best performance is "AMD-Normal" achieving a sensitivity and specificity greater than 0.95 . The rest of the experiments let show that the lower values correspond to the detection of the DR. This is because the lesions of the DR are usually smaller than those of the AMD and, therefore, they are more difficult to detect through texture analysis. Even so, the results are promising in the three-class diagnosis. 
Table 4.4: Results of the different experiments on the validation set.

\begin{tabular}{|c|c|c|c|c|c|c|}
\hline & \multicolumn{2}{|c|}{ AMD-Normal } & \multicolumn{2}{|c|}{ DR-Normal } & \multicolumn{2}{|c|}{ Path.-Normal } \\
\hline & TPR & TNR & TPR & TNR & TPR & TNR \\
\hline Logistic Regression & 1.000 & 0.920 & 0.412 & 0.960 & 0.815 & 0.920 \\
\hline Neural Networks & 1.000 & 1.000 & 0.647 & 0.960 & 0.704 & 0.960 \\
\hline SVM & 0.800 & 0.920 & 0.412 & 0.920 & 0.778 & 0.920 \\
\hline Naive Bayes & 1.000 & 0.960 & 0.353 & 0.920 & 0.556 & 0.800 \\
\hline $\mathrm{C} 4.5$ & 1.000 & 0.960 & 0.824 & 0.880 & 0.741 & 0.880 \\
\hline Rotation Forest & 0.900 & 0.920 & 0.706 & 0.840 & 0.815 & 0.880 \\
\hline Random Forest & 1.000 & 0.920 & 0.471 & 0.920 & 0.667 & 0.840 \\
\hline \multirow[t]{3}{*}{ AdaBoost } & 1.000 & 0.960 & 0.647 & 0.920 & 0.778 & 0.880 \\
\hline & & \multicolumn{2}{|c|}{$A M D-D R$} & \multicolumn{2}{|c|}{$A M D$-DR-Normal } & \\
\hline & & $\mathrm{TPR}$ & TNR & TPR & TNR & \\
\hline \multicolumn{2}{|c|}{ Logistic Regression } & 0.900 & 0.882 & 0.769 & 0.878 & \\
\hline \multicolumn{2}{|c|}{ Neural Networks } & 0.900 & 0.882 & 0.808 & 0.875 & \\
\hline \multicolumn{2}{|l|}{ SVM } & 1.000 & 0.824 & 0.750 & 0.812 & \\
\hline \multicolumn{2}{|l|}{ Naive Bayes } & 1.000 & 0.882 & 0.712 & 0.816 & \\
\hline \multicolumn{2}{|l|}{$\mathrm{C} 4.5$} & 1.000 & 0.882 & 0.808 & 0.874 & \\
\hline \multicolumn{2}{|c|}{ Rotation Forest } & 1.000 & 0.941 & 0.808 & 0.857 & \\
\hline \multicolumn{2}{|c|}{ Random Forest } & 1.000 & 0.824 & 0.808 & 0.892 & \\
\hline \multicolumn{2}{|l|}{ AdaBoost } & 0.900 & 0.824 & 0.769 & 0.886 & \\
\hline
\end{tabular}

Besides the results of the external CV, an independent set of 52 images (validation set) was saved to test the proposed method. The validation results follow the same trend shown in the CV, i.e. the "AMD-Normal" experiment is the one which achieves the best performance.

The proposed method was also compared with other state-of-the-art algorithms which are based on fundus image texture analysis (Garnier et al., 2014; Mookiah et al., 2013). As mentioned in the introduction, Garnier et al. use the LBP histogram calculated after a wavelet decomposition as input of a linear classifier validated by means of the leave-one-out method. Mookiah et al. combine the LBP information with other features (the area of hard exudates, the area of blood vessels, bifurcation points, etc.) in a probabilistic neural network. To the best 
of the author's knowledge, there is no other system that analyses the texture of the retina background and detects AMD and DR at the same time, therefore, it was only possible to compare the results from twoclass diagnosis, see Tables 4.5 and 4.6. The results of the proposed method shown in these tables are those achieved on the model set because the other compared works do not test their algorithms on an independent dataset. Note that the obtained results are not directly comparable because the dataset used in each case is different.

Table 4.5: AMD-Normal comparison on different databases.

\begin{tabular}{|c|c|c|c|c|c|c|}
\hline & TPR & TNR & Features & Classifier & Validation & \#images \\
\hline $\begin{array}{l}\text { Garnier et } \\
\text { al. }\end{array}$ & 0.913 & 0.955 & $\begin{array}{l}\text { Wavelet trans- } \\
\text { form and LBP }\end{array}$ & LDA & $\begin{array}{l}\text { Leave-one- } \\
\text { out }\end{array}$ & 45 \\
\hline $\begin{array}{l}\text { Proposed } \\
\text { method }\end{array}$ & 0.979 & 0.959 & $\mathrm{LBP}$ & AdaBoost & $\begin{array}{l}\text { 10-fold } \\
\text { external CV }\end{array}$ & 136 \\
\hline
\end{tabular}

Table 4.6: DR-Normal comparison on different databases.

\begin{tabular}{lccllllc}
\hline & TPR & TNR & Features & Classifier & Validation & \#images \\
\hline $\begin{array}{l}\text { Mookiah et } \\
\text { al. }\end{array}$ & 0.963 & 0.961 & $\begin{array}{l}\text { Blood vessel } \\
\text { area, exudates } \\
\text { area, bifurcation } \\
\text { points, texture } \\
\text { and entropy }\end{array}$ & $\begin{array}{l}\text { 3-fold } \\
\text { CV }\end{array}$ & 156 \\
& & & & RBP & $\begin{array}{l}\text { Random } \\
\text { Forest }\end{array}$ & $\begin{array}{l}\text { 10-fold } \\
\text { external } \\
\text { CV }\end{array}$ & 160 \\
$\begin{array}{l}\text { Proposed } \\
\text { method }\end{array}$ & 0.876 & 0.866 & & & & \\
\hline
\end{tabular}




\subsection{Conclusions}

In this chapter, a new approach for AMD and DR diagnosis has been presented. It is based on analyzing texture discrimination capabilities in fundus images to discriminate healthy patients from images with AMD or DR. The performance of LBP with different classifiers was tested.

Many more AMD or DR detection techniques exist in the literature but most of them focus on lesion segmentation instead of a direct study of the retina background. This fact makes the accuracy of the classification stage dependent on the accuracy of the lesion segmentation. Lesion segmentation involves a series of uncertainties and a non-accurate segmentation may provoke important errors in the classification. The main advantage of the proposed procedure is that it gets a good performance without having to search different types of lesions. The only segmentation needed in the presented approach is to mask the significant structures (vessels and optic disk) but their accuracy has little influence on the final result.

The obtained results, a sensitivity and specificity greater than 0.96 for AMD detection, greater than 0.87 for DR detection and greater than 0.91 for three-class diagnosis, demonstrate that LBP is a good texture descriptor for fundus images and can be used in a diagnosis aid system for retinal disease screening.

As for future work lines, more images could be added to the database and the analysis could be repeated. Moreover, some work should be carried out to develop strategies that enable the analysis of the type of images that were excluded from the initial database, such as tessellated fundus, images with highlights or typical artefacts. 



\section{Chapter 5}

\section{Conclusions}

As was indicated in Chapter 1, the main objective of this thesis was to develop and implement algorithms for retina characterization from the analysis of fundus images. In particular, the retina was characterized through advanced image processing techniques in two different ways. First, the main retinal structures, blood vessels and optic disk, were detected and subsequently analyzed to obtain some of their significant features. This information was used in a computer-aided diagnosis software for hypertensive risk determination. This was addressed in Chapter 3. Secondly, the texture of the retina background was studied in order to classify an image as belonging to a healthy or pathological subject, as was seen in Chapter 4 . Diabetic retinopathy and agerelated macular degeneration were detected by this procedure. Its main advantage is to avoid the need for segmentation of each type of retinal lesion.

On the one hand, Chapter 3 demonstrated that the performance of mathematical morphology and stochastic watershed, a variant of the watershed transformation, allows us to apply these methods for clinical purposes as the segmentation of the retinal structures. On the 
other hand, Chapter 4 stood out that local binary pattern operator is a good texture descriptor for fundus images and can be used for retinal disease screening, above all for the diagnosis of age-related macular degeneration.

The methods presented in this thesis will be incorporated in an only automatic screening system to discriminate between a normal and pathological retina taking into account different pathologies at the same time. This system will have to be more robust than the existing solutions and to have enough specificity and sensitivity to be applied in clinical practice. Nowadays, there are some initiatives focused on glaucoma or diabetic retinopathy detection but they have very low specificity and, therefore, low screening effectiveness. The development of this screening system is being funded at the present time by the Spanish government under the project ACRIMA (TIN2013-46751-R). It is expected to have a first version of the system at the end of 2016. In this project, new prognostic markers that are currently used in clinical diagnosis, such as blood pressure, age or family history, will be combined with those provided by the fundus image processing with the aim of improving the robustness and increasing the sensitivity and specificity of the automatic screening system. From a perspective of a screening tool, all developments presented in this thesis should be validated by comparing the results achieved by the tool with the diagnosis made by an expert.

In future works, three main research lines will be carried out. First, to segment other retinal structures such as the macula or the optic cup. The location of the macula is important for the diagnosis of age-related macular degeneration, as its name suggests. The area of the optic cup is directly related to glaucoma prediction, as was seen in Chapter 2. Secondly, to perform an in-depth study of the retina background. More texture descriptors will be evaluated and their results will be compared with those presented in Chapter 4. Finally, to consider the heterogeneity of the clinical databases. The parameters used in the developed methods which are related to the image size will be generalized. The diverse 
types of normality that exist in fundus images, as the tessellated images, will have to be taken into account to not reduce the specificity of the classification, which is increasingly important. 



\section{Merits}

\section{Journal papers}

Morales, S., Naranjo, V., Angulo, J., Alcañiz, M. (2013). Automatic detection of optic disc based on PCA and mathematical morphology. IEEE Transactions on Medical Imaging, 32(4): 786796.

Morales, S., Naranjo, V., Navea, A., Alcañiz, M. (2014). Computer-Aided Diagnosis Software for Hypertensive Risk Determination Through Fundus Image Processing. IEEE Journal of Biomedical and Health Informatics, 18(6):1757-1763 (Retitled from the IEEE Transactions on Information Technology in Biomedicine (T-ITB) in 2013).

Morales, S., Engan, K., Naranjo, V., Colomer, A. (2015). Retinal Disease Screening through Local Binary Patterns. IEEE Journal of Biomedical and Health Informatics (Under revision).

\section{International conferences}

Morales, S., Naranjo, V., Angulo, J., Fuertes, J. J., Alcañiz, M. (2012). Segmentation and Analysis of Retinal Vascular Tree from Fundus Images Processing. In International Conference on 
Bio-inspired Systems and Signal Processing (BIOSIGNALS 2012), pages 321-324.

Morales, S., Naranjo, V., Pérez, D., Navea, A., Alcañiz, M. (2012). Automatic detection of optic disc based on PCA and stochastic watershed. In European Signal Processing Conference (EUSIPCO 2012), pages 2605-2609.

Morales, S., Naranjo, V., Angulo, J., Navea, A., Alcañiz, M. (2013). Characterization and Measurement of Retinal Vascular Tree through Fundus Image Processing. In International Conference on Medical Imaging using Bio-inspired and Soft Computing (MIBISOC 2013), pages 99-105.

Morales, S., Naranjo, V., López-Mir, F., Navea, A., Alcañiz, M. (2013). Computer-Assisted System for Hypertensive Risk Determination through Fundus Image Processing. In XIII Mediterranean Conference on Medical and Biological Engineering and Computing 2013, volume 41 of IFMBE Proceedings, pages 273276 .

Montoro, A., Morales, S., Naranjo, V., López-Mir, F., Alcañiz, M. (2014). Feature extraction for retinal vascular network classification. In 2nd IEEE-EMBS International Conference on Biomedical and Health Informatics (BHI 2014), pages 404-407.

López, F., Ureña, N., Ruiz, J. L., Naranjo, V., Morales, S.. ImageAssisted System for the Diagnosis of Bladder Tumor Recurrence. 2nd IEEE-EMBS International Conference on Biomedical and Health Informatics (BHI 2014), 2014.

Morales, S., Naranjo, V., Angulo, J., López-Mir, F., Alcañiz, M. (2014). Determination of Retinal Network Skeleton through Mathematical Morphology. In 22nd European Signal Processing Conference (EUSIPCO 2014), pages 1691-1695. 
López-Mir, F., Naranjo, V., Morales, S., Angulo, J. Probability density function of object contours using regional regularized stochastic watershed. In IEEE International Conference on Image Processing (ICIP 2014), 4762-4766, 2014.

Morales, S., Legaz-Aparicio, A. G., Naranjo, V., Verdú-Monedero, R. (2015). Determination of Bifurcation Angles of the Retinal Vascular Tree through Multiple Orientation Estimation based on Regularized Morphological Openings. In 8th International Conference on Bio-inspired Systems and Signal Processing (BIOSIGNALS 2015), pages 126-131.

Morales, S., Engan, K., Naranjo, V., Colomer, A. (2015). Detection of Diabetic Retinopathy and Age-related Macular Degeneration from Fundus Images through Local Binary Patterns and Random Forests. In IEEE International Conference on Image Processing (ICIP 2015) (Accepted for publication).

Morales, S., Naranjo, V., Colomer, A., Alcañiz, M. (2015). Significant point characterization in fundus images. In 5th International Conference on Image Processing Theory, Tools and Applications (IPTA 2015) (Accepted for publication).

\section{National conferences}

Morales, S., Naranjo, V., Alcón, J., Alcañiz, M., Lurbe, E. (2010). Herramienta software para el estudio de la arquitectura microvascular retiniana mediante técnicas de tratamiento digital de imagen de fondo de ojo. In XXVIII Congreso Anual de la Sociedad Española de Ingeniería Biomédica (CASEIB 2010), pages 20-23.

Pérez, D., Morales, S., Naranjo, V., Navea, A., Alcañiz, M. (2011). Detección automática del disco óptico mediante la transformada watershed estocástica. In XXIX Congreso Anual de la Sociedad Española de Ingeniería Biomédica (CASEIB 2011), pages 63-66. 
Rodrigo, B., Morales, S., Naranjo, V., Colomer, A., Alcañiz, M. (2014). Detección de glaucoma mediante la combinación de la relación copa/disco y la regla ISNT. In XXXII Congreso Anual de la Sociedad Española de Ingeniería Biomédica (CASEIB 2014).

\section{Book chapters}

Morales, S., Naranjo, V., Alcañiz, M. (2014). Automatic Detection of Retinal Structures Based on Mathematical Morphology, chapter 11 of Frontiers of Medical Imaging, pages 211-232. World Scientific Publishing.

\section{Research awards}

Morales, S. (2013). Técnicas avanzadas de análisis de imagen aplicadas a la segmentación y caracterización del árbol vascular retiniano. Accèsit en el VIIIé Premi d'Investigació CientíficoTècnica "Ciutat d'Algemesí" per a joves investigadors.

López-Mir, F., Naranjo, V., Morales, S., Angulo, J. (2014). Probability density function of object contours using regional regularized stochastic watershed. Top 10\% Paper Award in the IEEE International Conference on Image Processing (ICIP 2014). 


\section{Bibliography}

Abràmoff, M., Garvin, M., and Sonka, M. (2010). Retinal imaging and image analysis. Biomedical Engineering, IEEE Reviews in, 3:169-208. 3 citations in pages 3,15 , and 80 .

Abràmoff, M. D., Niemeijer, M., Suttorp-Schulten, M. S., Viergever, M. A., Russell, S. R., and van Ginneken, B. (2008). Evaluation of a system for automatic detection of diabetic retinopathy from color fundus photographs in a large population of patients with diabetes. Diabetes Care, 31(2):193-198. One citation in page 20.

Abràmoff, M. D., Reinhardt, J. M., Russell, S. R., Folk, J. C., Mahajan, V. B., Niemeijer, M., and Quellec, G. (2010). Automated early detection of diabetic retinopathy. Ophthalmology, 117(6):1147 - 1154. One citation in page 79 .

Agurto, C., Barriga, E. S., Murray, V., Nemeth, S., Crammer, R., Bauman, W., Zamora, G., Pattichis, M. S., and Soliz, P. (2011a). Automatic detection of diabetic retinopathy and age-related macular degeneration in digital fundus images. Investigative Ophthalmology 83 Visual Science, 52(8):5862-5871. 2 citations in pages 21 and 24.

Agurto, C., Barriga, E. S., Zamora, G., Murray, V., Murillo, S., Yu, H., Bauman, Wendall C., J., and Soliz, P. (2011b). Automatic screening of eye diseases using 3-field fundus photographs. Investigative Ophthalmology \& Visual Science, 52(6):1342. One citation in page 24.

Ahonen, T., Hadid, A., and Pietikainen, M. (2006). Face description with local binary patterns: Application to face recognition. Pattern Analysis and Machine Intelligence, IEEE Transactions on, 28(12):2037-2041. One citation in page 120 . 
Al-Diri, B. and Hunter, A. (2009). Automated measurements of retinal bifurcations. In Dössel, O. and Schlegel, W., editors, World Congress on Medical Physics and Biomedical Engineering, September 7 - 12, 2009, Munich, Germany, volume 25/11 of IFMBE Proceedings, pages 205-208. Springer Berlin Heidelberg. One citation in page 23.

Al-Diri, B., Hunter, A., and Steel, D. (2009). An active contour model for segmenting and measuring retinal vessels. Medical Imaging, IEEE Transactions on, 28(9):1488-1497. One citation in page 23.

Al-Rawi, M., Qutaishat, M., and Arrar, M. (2007). An improved matched filter for blood vessel detection of digital retinal images. Computers in Biology and Medicine, 37(2):262-267. One citation in page 32.

American Diabetes Association (2008). Economic costs of diabetes in the u.s. in 2007. Diabetes Care, 31(3):596-615. One citation in page 4.

Angulo, J. and Jeulin, D. (2007). Stochastic watershed segmentation. In Proc. of the 8th International Symposium on Mathematical Morphology (ISMM'2007), pages 265-279. 2 citations in pages 42 and 76.

Angulo, J. and Velasco-Forero, S. (2010). Semi-supervised hyperspectral image segmentation using regionalized stochastic watershed. Proc. SPIE, Algorithms and Technologies for Multispectral, Hyperspectral, and Ultraspectral Imagery XVI, 7695:76951F-76951F-12. One citation in page 42.

Anitha, J., C. Kezi Selva Vijila, and D. Jude Hemanth (2011). An overview of computational intelligence techniques for retinal disease identification applications. International Journal of Reviews in Computing, 5:29-46. One citation in page 3 .

Aquino, A., Gegúndez-Arias, M. E., and Marín, D. (2010). Detecting the optic disc boundary in digital fundus images using morphological, edge detection, and feature extraction techniques. Medical Imaging, IEEE Transactions on, 29(11):1860 - 1869. 5 citations in pages 25, 33, 78, 108, and 109 .

Azzopardi, G. and Petkov, N. (2011). Detection of retinal vascular bifurcations by trainable v4-like filters. In Real, P., Diaz-Pernil, D., Molina-Abril, H., Berciano, A., and Kropatsch, W., editors, Computer Analysis of Images and Patterns, volume 6854 of Lecture Notes in Computer Science, pages 451-459. Springer Berlin Heidelberg. One citation in page 32. 
Bernander, K. B., Gustavsson, K., Selig, B., Sintorn, I.-M., and Luengo Hendriks, C. L. (2013). Improving the stochastic watershed. Pattern Recognition Letters, 34(9):993 - 1000. One citation in page 42.

Bessaid, A., Feroui, A., and Messadi, M. (2009). Detection of blood vessels from retinal images using watershed transformation. Journal of Mechanics in Medicine and Biology, 09(04):633-642. 4 citations in pages $32,95,96$, and 97 .

Beucher, S. and Meyer, F. (1992). The morphological approach to segmentation: The watershed transformation. In Mathematical Morphology in Image Processing, chapter 12. E. R. Dougherty. 2 citations in pages 39 and 42 .

Bevilacqua, V., Cambò, S., Cariello, L., and Mastronardi, G. (2005). A combined method to detect retinal fundus features. In Proceedings of IEEE European Conference on Emergent Aspects in Clinical Data Analysis, pages 1-6. One citation in page 32 .

Bhuiyan, A., Nath, B., Chua, J., and Ramamohanarao, K. (2007). Automatic detection of vascular bifurcations and crossovers from color retinal fundus images. In Signal-Image Technologies and Internet-Based System, $200 \%$. SITIS '07. Third International IEEE Conference on, pages 711-718. One citation in page 32 .

Breiman, L. (2001). Random forests. Machine Learning, 45(1):5-32. One citation in page 135 .

Calvo, D., Ortega, M., Penedo, M. G., and Rouco, J. (2011). Automatic detection and characterisation of retinal vessel tree bifurcations and crossovers in eye fundus images. Computer Methods and Programs in Biomedicine, 103(1):28 - 38. 2 citations in pages 32 and 56.

Carmona, E. J., Rincón, M., García-Feijoó, J., and Martínez-de-la-Casa, J. M. (2008). Identification of the optic nerve head with genetic algorithms. Artif. Intell. Med., 43:243-259. 2 citations in pages 87 and 98.

Cessie, L. S. and van Houwelingen, J. C. (1992). Ridge Estimators in Logistic Regression. Applied Statistics, 41(1):191-201. One citation in page 135.

Chalana, V., Linker, D. T., Haynor, D. R., and Kim, Y. (1996). A multiple active contour model for cardiac boundary detection on echocardiographic sequences. IEEE Transactions on Medical Imaging, 15(3):290-298. One citation in page 97 . 
Chang, C. C. and Lin, C. J. (2011). LIBSVM: A library for support vector machines. Intelligent Systems and Technology, ACM Transactions on, $2(3): 27: 1-27: 27$. One citation in page 135.

Chaudhuri, S., Chatterjee, S., Katz, N., Nelson, M., and Goldbaum, M. (1989). Automatic detection of the optic nerve in retinal images. In IEEE International Conference on Image Processing, volume 1, pages 1-5. 2 citations in pages 90 and 92 .

Chawla, N. V., Bowyer, K. W., Hall, L. O., and Kegelmeyer, W. P. (2002). SMOTE: Synthetic minority over-sampling technique. Artificial Intelligence Research, Journal of, 16:321-357. One citation in page 127.

Chen, L., Ju, Y., Ding, S., and Liu, X. (2011). Topological vascular tree segmentation for retinal images using shortest path connection. In Image Processing (ICIP), 2011 18th IEEE International Conference on, pages 2137-2140. One citation in page 32 .

Cheung, N. and Wong, T. Y. (2008). Diabetic retinopathy and systemic vascular complications. Progress in Retinal and Eye Research, 27(2):161 - 176. One citation in page 79.

Chilès, J.-P. and Delfiner, P. (2012). Geostatistics : modeling spatial uncertainty. Wiley Series in Probability and Statistics. John Wiley \& Sons, Inc., New York, 2nd edition. One citation in page 42.

Corral, C. A. and Lindquist, C. S. (1998). On implementing kasa's circle fit procedure. IEEE Transactions on Instrumentation and Measurement, 47(3):789-795. One citation in page 79.

Cree, M. J. and Jelinek, H. F. (2011). Image analysis of retinal images. In Dougherty, G., editor, Medical Image Processing: Techniques and Applications, Biological and Medical Physics, Biomedical Engineering, chapter 11, pages 249-268. Springer. 2 citations in pages 3 and 44 .

Decencière, E., Cazuguel, G., Zhang, X., Thibault, G., Klein, J.-C., Meyer, F., Marcotegui, B., Quellec, G., Lamard, M., Danno, R., Elie, D., Massin, P., Viktor, Z., Erginay, A., Laÿ, B., and Chabouis, A. (2013). TeleOphta: Machine learning and image processing methods for teleophthalmology. IRBM (Special issue: ANR TECSAN: Technologies for Health and Autonomy), 34(2):196-203. One citation in page 131.

Dhanushkodi, S. and Vasuki, M. (2013). Diagnosis system for diabetic retinopathy to prevent vision loss. Applied Medical Informatics, 33(3):111. One citation in page 120 . 
Duda, R. O. and Hart, P. E. (1973). Pattern Classification and Scene Analysis. John Wiley \& Sons Inc. One citation in page 43.

Dudoit, S. and van der Laan, M. J. (2005). Asymptotics of cross-validated risk estimation in estimator selection and performance assessment. Statistical Methodology, 2(2):131 - 154. One citation in page 127.

Early Treatment DR Study Research Group (1991). Grading diabetic retinopathy from stereoscopic color fundus photographs - an extension of the modified airlie house classification (ETDRS) report number 10 . Ophthalmology, 98(5, Supplement):786 - 806. One citation in page 18 .

Estabridis, K. and de Figueiredo, R. J. (2007). Automatic detection and diagnosis of diabetic retinopathy. In Image Processing, 200\%. ICIP $200 \%$. IEEE International Conference on, volume 2, pages II - 445-II - 448. One citation in page 20 .

Eswaran, C., Reza, A., and Hati, S. (2008). Extraction of the contours of optic disc and exudates based on marker-controlled watershed segmentation. In Computer Science and Information Technology, 2008. ICCSIT '08. International Conference on, pages $719-723.2$ citations in pages 33 and 109.

Farnell, D., Hatfield, F., Knox, P., Reakes, M., Spencer, S., Parry, D., and Harding, S. (2008). Enhancement of blood vessels in digital fundus photographs via the application of multiscale line operators. Franklin Institute, Journal of the, 345(7):748 - 765. One citation in page 130 .

Faust, O., Acharya U., R., Ng, E., Ng, K.-H., and Suri, J. (2012). Algorithms for the automated detection of diabetic retinopathy using digital fundus images: A review. Journal of Medical Systems, 36(1):145-157. One citation in page 20 .

Fong, D. S., Aiello, L., Gardner, T. W., King, G. L., Blankenship, G., Cavallerano, J. D., Ferris, F. L., and Klein, R. (2003). Diabetic retinopathy. Diabetes Care, 26(1):226-229. One citation in page 18.

Fraz, M., Remagnino, P., Hoppe, A., Uyyanonvara, B., Rudnicka, A., Owen, C., and Barman, S. (2012). Blood vessel segmentation methodologies in retinal images: A survey. Computer Methods and Programs in Biomedicine, 108(1):407 - 433. 2 citations in pages 31 and 32.

Freund, Y. and Schapire, R. E. (1996). Experiments with a new boosting algorithm. In Saitta, L., editor, Machine Learning, Proceedings of the 
Thirteenth International Conference on (ICML 1996), pages 148-156. Morgan Kaufmann. One citation in page 135.

Gagnon, L., Lalonde, M., Beaulieu, M., and Boucher, M. C. (2001). Procedure to detect anatomical structures in optical fundus images. Proceedings of SPIE, 4322:1218-1225. One citation in page 65.

Gao, X. W., Bharath, A., Stanton, A., Hughes, A., Chapman, N., and Thom, S. (2000). Quantification and characterisation of arteries in retinal images. Computer Methods and Programs in Biomedicine, 63(2):133 - 146. One citation in page 33 .

Garnier, M., Hurtut, T., Ben Tahar, H., and Cheriet, F. (2014). Automatic multiresolution age-related macular degeneration detection from fundus images. In SPIE, Proceedings, volume 9035, pages 903532-903532-7. 4 citations in pages 21, 120,131, and 137.

Goatman, K., Charnley, A., Webster, L., and Nussey, S. (2011). Assessment of automated disease detection in diabetic retinopathy screening using two-field photography. PLoS ONE, 6(12, e27524). 2 citations in pages 24 and 79 .

Gonzalez, R. C., Woods, R. E., and Eddins, S. L. (2004). Digital Image Processing using MATLAB. Pearson Prentice Hall. 2 citations in pages 33 and 57.

Grisan, E., Pesce, A., Giani, A., Foracchia, M., and Ruggeri, A. (2004). A new tracking system for the robust extraction of retinal vessel structure. In Engineering in Medicine and Biology Society, 2004. IEMBS '04. 26th Annual International Conference of the IEEE, volume 1, pages 16201623. One citation in page 32 .

Guillemot, C. and Le Meur, O. (2014). Image inpainting: Overview and recent advances. IEEE Signal Processing Magazine, 31(1):127-144. One citation in page 69 .

Hajer, J., Kamel, H., and Noureddine, E. (2008). Localization of the optic disk in retinal image using the "watersnake". In Computer and Communication Engineering, 2008. ICCCE 2008. International Conference on, pages 947 -951. 2 citations in pages 33 and 109.

Hall, M., Frank, E., Holmes, G., Pfahringer, B., Reutemann, P., and Witten, I. H. (2009). The weka data mining software: An update. SIGKDD Explor. Newsl., 11(1):10-18. One citation in page 130. 
Hatanaka, Y., Noudo, A., Muramatsu, C., Sawada, A., Hara, T., Yamamoto, T., and Fujita, H. (2010). Automatic measurement of vertical cup-todisc ratio on retinal fundus images. In Medical Biometrics, volume 6165 of Lecture Notes in Computer Science, pages 64-72. Springer Berlin / Heidelberg. 3 citations in pages 23, 31, and 64 .

Hecht-Nielsen, R. (1990). Neurocomputing. Addison-Wesley Longman Publishing Co., Inc. One citation in page 135.

Heikkil M. (2009). Description of interest regions with local binary patterns. Pattern Recognition, 42(3):425 - 436. One citation in page 120.

Herbert, H. M., Jordan, K., and Flanagan, D. W. (2003). Is screening with digital imaging using one retinal view adequate? Eye, (4):497-500. One citation in page 3 .

Hijazi, M. H. A., Coenen, F., and Zheng, Y. (2012). Data mining techniques for the screening of age-related macular degeneration. Know.-Based Syst., 29:83-92. One citation in page 21 .

Hoover, A. and Goldbaum, M. (2003). Locating the optic nerve in a retinal image using the fuzzy convergence of the blood vessels. Medical Imaging, IEEE Transactions on, 22(8):951 -958. One citation in page 131.

Hoover, A., Kouznetsova, V., and Goldbaum, M. (2000). Locating blood vessels in retinal images by piecewise threshold probing of a matched filter response. IEEE Transactions on Medical Imaging, 19:203-210. 4 citations in pages $32,86,94$, and 131 .

Hsu, W., Lee, M. L., and Wong, T. Y. (2011). Retinal image analysis systems and methods. Patent US 2011/0026789 A1. http://scholarbank.nus.edu.sg/bitstream/handle/10635/ 34962/US20110026789A1. PDF? sequence=1. Last access on 27th December 2014. One citation in page 24 .

Hubbard, L. D., Brothers, R. J., King, W. N., Clegg, L. X., Klein, R., Cooper, L. S., Sharrett, A., Davis, M. D., and Cai, J. (1999). Methods for evaluation of retinal microvascular abnormalities associated with hypertension/sclerosis in the atherosclerosis risk in communities study. Ophthalmology, 106(12):2269 - 2280. 3 citations in pages 17, 64, and 81 .

Jager, R. D., Mieler, W. F., and Miller, J. W. (2008). Age-related macular degeneration. New England Journal of Medicine, 358(24):2606-2617. One citation in page 20 . 
Jiang, X. and Mojon, D. (2003). Adaptive local thresholding by verificationbased multithreshold probing with application to vessel detection in retinal images. IEEE Trans. Pattern Anal. Mach. Intell., 25:131-137. 3 citations in pages 32,90 , and 92 .

John, G. H. and Langley, P. (1995). Estimating continuous distributions in bayesian classifiers. In Uncertainty in Artificial Intelligence, Proceedings of the Eleventh Conference on, UAI'95, pages 338-345. Morgan Kaufmann Publishers Inc. One citation in page 135.

Jolliffe, I. T. (2002). Principal Component Analysis. Springer, second edition. One citation in page 67 .

Kahai, P., Namuduri, K. R., and Thompson, H. (2006). A decision support framework for automated screening of diabetic retinopathy. Int. J. Biomedical Imaging, 2006. One citation in page 20.

Kande, G. B., Subbaiah, P. V., and Savithri, T. S. (2010). Unsupervised fuzzy based vessel segmentation in pathological digital fundus images. Journal of medical systems, 34(5):849-858. One citation in page 32 .

Kanski, J. and Bowling, B. (2011). Clinical Ophthalmology: A Systematic Approach, 7th Edition. Elsevier Health Sciences. One citation in page 31.

Kauppi, T., Kalesnykiene, V., Kamarainen, J.-K., Lensu, L., Sorri, I., A. Raninen, A., Voutilainen, R., Uusitalo, H., Kälviäinen, H., and Pietilä, J. (2007). The DIARETDB1 diabetic retinopathy database and evaluation protocol. In British Machine Vision Conference (BMVC2007), pages 252-261. 2 citations in pages 87 and 98 .

Kauppi, T. and Kälviäinen, H. (2008). Simple and robust optic disc localisation using colour decorrelated templates. In Proceedings of the 10th International Conference on Advanced Concepts for Intelligent Vision Systems, ACIVS '08, pages 719-729, Berlin, Heidelberg. SpringerVerlag. One citation in page 33.

Knudtson, M. D., Lee, K. E., Hubbard, L. D., Wong, T. Y. Y., Klein, R., and Klein, B. E. (2003). Revised formulas for summarizing retinal vessel diameters. Current Eye Research, 27(3):143-149. 3 citations in pages 17, 64 , and 81.

Kohavi, R. and John, G. H. (1997). Wrappers for feature subset selection. Artificial Intelligence, 97(1):273 - 324. One citation in page 128. 
Kotu, L., Engan, K., Eftestol, T., Woie, L., Orn, S., and Katsaggelos, A. (2012). Local binary patterns used on cardiac MRI to classify high and low risk patient groups. In Signal Processing Conference (EUSIPCO), Proceedings of the 20th European, pages 2586-2590. One citation in page 120.

Kwon, Y. H., Fingert, J. H., Kuehn, M. H., and Alward, W. L. (2009). Primary open-angle glaucoma. New England Journal of Medicine, 360(11):11131124. One citation in page 21.

Lalonde, M., Beaulieu, M., and Gagnon, L. (2001). Fast and robust optic disc detection using pyramidal decomposition and hausdorff-based template matching. Medical Imaging, IEEE Transactions on, 20(11):1193 -1200. 3 citations in pages 33, 78, and 109.

Levin, A., Zomet, A., and Weiss, Y. (2003). Learning how to inpaint from global image statistics. In Computer Vision, 2003. Proceedings. Ninth IEEE International Conference on, pages 305-312 vol.1. One citation in page 70 .

Li, H. and Chutatape, O. (2004). Automated feature extraction in color retinal images by a model based approach. Biomedical Engineering, IEEE Transactions on, 51(2):246 -254. One citation in page 33 .

Liang, L., Liu, C., Xu, Y., Guo, B., and yeung Shum, H. (2001). Realtime texture synthesis by patch-based sampling. ACM Transactions on Graphics, 20:127-150. One citation in page 70 .

Liao, S., Law, M., and Chung, A. (2009). Dominant local binary patterns for texture classification. Image Processing, IEEE Transactions on, 18(5):1107-1118. One citation in page 120.

Liew, G. and Wang, J. J. (2011). Retinal vascular signs: A window to the heart? Revista Española de Cardiología (English Edition), 64(6):515 521. One citation in page 17.

Liu, J., Wong, D. W. K., Lim, J. H., Li, H., Tan, N. M., Zhang, Z., Wong, T. Y., and Lavanya, R. (2009). Argali: An automatic cupto-disc ratio measurement system for glaucoma analysis using levelset image processing. In 13th International Conference on Biomedical Engineering, volume 23 of IFMBE Proceedings, pages 559-562. Springer Berlin Heidelberg. 3 citations in pages 23, 24, and 64 . 
Lowell, J., Hunter, A., Steel, D., Basu, A., Ryder, R., Fletcher, E., and Kennedy, L. (2004). Optic nerve head segmentation. IEEE Trans. Med. Imaging, 23(2):256-264. 7 citations in pages 23, 33, 66, 88, 98, 108, and 109 .

Lu, S. (2011). Accurate and efficient optic disc detection and segmentation by a circular transformation. Medical Imaging, IEEE Transactions on, 30(12):2126 -2133. One citation in page 109.

MacGillivray, T., Perez-Rovira, A., Trucco, E., Chin, K., Giachetti, A., Lupascu, C., Tegolo, D., Wilson, P., Doney, R., Laude, A., and Dhillon, B. (2012). VAMPIRE: Vessel Assessment and Measurement Platform for Images of the Retina. CRC Press. One citation in page 33.

MacQueen, J. B. (1967). Some methods for classification and analysis of multivariate observations. In Proc. of the fifth Berkeley Symposium on Mathematical Statistics and Probability, volume 1, pages 281-297. One citation in page 49 .

Mairal, J., Bach, F., Ponce, J., Sapiro, G., and Zisserman, A. (2008). Discriminative learned dictionaries for local image analysis. In $C V P R$. IEEE Computer Society. One citation in page 70.

Marín, D., Aquino, A., Gegúndez-Arias, M. E., and Bravo, J. M. (2011). A new supervised method for blood vessel segmentation in retinal images by using gray-level and moment invariants-based features. Medical Imaging, IEEE Transactions on, 30(1):146-158. 2 citations in pages 25 and 32.

Martinez-Perez, M., Hughes, A., Stanton, A., Thom, S., Bharath, A., and Parker, K. (1999). Retinal blood vessel segmentation by means of scalespace analysis and region growing. In Medical Image Computing and Computer-Assisted Intervention MICCAI 99, volume 1679 of Lecture Notes in Computer Science, pages 90-97. 2 citations in pages 90 and 92.

Martinez-Perez, M. E., Hughes, A. D., Stanton, A. V., Thorn, S. A., Chapman, N., Bharath, A. A., and Parker, K. H. (2002). Retinal vascular tree morphology: A semi-automatic quantification. In Biomedical Engineering, volume 49. 2 citations in pages 33 and 82 .

Martinez-Perez, M. E., Hughes, A. D., Thom, S. A., Bharath, A. A., and Parker, K. H. (2007). Segmentation of blood vessels from red-free and fluorescein retinal images. Medical Image Analysis, 11:47-61. 7 citations in pages $32,47,52,92,95,96$, and 97 . 
Massey, E. M., Lowell, J., Hunter, A., and Steel, D. (2009). Lesion boundary segmentation using level set methods. In Ranchordas, A. and Ara $\tilde{A}^{\circ}$ jo, H., editors, VISAPP - Proceedings of the Fourth International Conference on Computer Vision Theory and Applications, pages 245-249. INSTICC Press. One citation in page 23.

Mendonça, A. and Campilho, A. (2006). Segmentation of retinal blood vessels by combining the detection of centerlines and morphological reconstruction. Medical Imaging, IEEE Transactions on, 25(9):12001213. 2 citations in pages 32 and 92.

Miller, J. W. (2013). Age-related macular degeneration revisited - Piecing the puzzle - The LXIX Edward Jackson memorial lecture. American Journal of Ophthalmology, 155(1):1 - 35.e13. One citation in page 21.

Mookiah, M., Acharya, U. R., Martis, R. J., Chua, C. K., Lim, C., Ng, E., and Laude, A. (2013). Evolutionary algorithm based classifier parameter tuning for automatic diabetic retinopathy grading: A hybrid feature extraction approach. Knowledge-Based Systems, 39(0):9-22. 2 citations in pages 120 and 137 .

Morales, S., Naranjo, V., Angulo, J., and Alcañiz, M. (2013). Automatic detection of optic disc based on pca and mathematical morphology. Medical Imaging, IEEE Transactions on, 32(4):786-796. 2 citations in pages 5 and 82 .

Morales, S., Naranjo, V., Angulo, J., Fuertes, J. J., and Alcañiz, M. (2012). Segmentation and analysis of retinal vascular tree from fundus images processing. In International Conference on Bio-inspired Systems and Signal Processing (BIOSIGNALS 2012), pages 321 - 324. 5 citations in pages $5,82,95,96$, and 97 .

Morales, S., Naranjo, V., Angulo, J., López-Mir, F., and Alcañiz, M. (2014a). Determination of retinal network skeleton through mathematical morphology. In EUSIPCO 2014 (22nd European Signal Processing Conference 2014), Lisbon, Portugal. One citation in page 5.

Morales, S., Naranjo, V., Navea, A., and Alcaniz, M. (2014b). Computer-aided diagnosis software for hypertensive risk determination through fundus image processing. Biomedical and Health Informatics, IEEE Journal of, 18(6):1757-1763. One citation in page 6 .

Muramatsu, C., Hatanaka, Y., Iwase, T., Hara, T., and Fujita, H. (2010). Automated detection and classification of major retinal vessels for 
determination of diameter ratio of arteries and veins. volume 7624 , pages 76240J-76240J-8. 2 citations in pages 17 and 80.

Nanni, L., Lumini, A., and Brahnam, S. (2010). Local binary patterns variants as texture descriptors for medical image analysis. Artificial Intelligence in Medicine, 49(2):117 - 125. One citation in page 120.

Nath, M. K. and Dandapat, S. (2012). Techniques of glaucoma detection from color fundus images: A review. International Journal of Image, Graphics and Signal Processing, 4(9):44-51. 2 citations in pages 22 and 23.

Nayak, J., Acharya U., R., Bhat, P., Shetty, N., and Lim, T.-C. (2009). Automated diagnosis of glaucoma using digital fundus images. Journal of Medical Systems, 33(5):337-346. One citation in page 23.

Nguyen, T. T., Wang, J. J., and Wong, T. Y. (2007). Retinal vascular changes in pre-diabetes and prehypertension: New findings and their research and clinical implications. Diabetes Care, 30(10):2708-2715. One citation in page 79 .

Niemeijer, M., Abràmoff, M. D., and van Ginneken, B. (2009). Fast detection of the optic disc and fovea in color fundus photographs. Medical Image Analysis, 13(6):859 - 870. 6 citations in pages 31, 33, 64, 66, 78, and 109.

Niemeijer, M., Staal, J., van Ginneken, B., Loog, M., and Abramoff, M. (2004). Comparative study of retinal vessel segmentation methods on a new publicly available database. In SPIE Medical Imaging, volume 5370, pages 648-656. 2 citations in pages 90 and 92.

Niemeijer, M., Xu, X., Dumitrescu, A., Gupta, P., van Ginneken, B., Folk, J., and Abramoff, M. (2011). Automated measurement of the arteriolar-tovenular width ratio in digital color fundus photographs. Medical Imaging, IEEE Transactions on, 30(11):1941-1950. 2 citations in pages 17 and 80 .

Ojala, T., Pietikäinen, M., and Mäenpää, T. (2001). A generalized local binary pattern operator for multiresolution gray scale and rotation invariant texture classification. In in Proc. of Second Inter. Conf. on Advances in Pattern Recognition, Rio de Janeiro, pages 397-406. 2 citations in pages 120 and 121 .

Ojala, T., Pietikäinen, M., and Maenpää, T. (2002). Multiresolution grayscale and rotation invariant texture classification with local binary patterns. Pattern Analysis and Machine Intelligence, IEEE Transactions on, 24(7):971-987. 2 citations in pages 120 and 121. 
Oppedal, K., Engan, K., Aarsland, D., Beyer, M., Tysnes, O. B., and Eftestol, T. (2012). Using local binary pattern to classify dementia in MRI. In Biomedical Imaging (ISBI), 9th IEEE International Symposium on, pages 594-597. One citation in page 120.

Ortega, M., Barreira, N., Novo, J., Penedo, M., Pose-Reino, A., and GómezUlla, F. (2010). Sirius: A web-based system for retinal image analysis. International Journal of Medical Informatics, 79(10):722 - 732. 3 citations in pages 17,25 , and 80 .

Osareh, A., Mirmehdi, M., Thomas, B., and Markham, R. (2002). Comparison of colour spaces for optic disc localisation in retinal images. In Pattern Recognition, 2002. Proceedings. 16th International Conference on, volume 1, pages 743 - 746 vol.1. 3 citations in pages 33, 66, and 109 .

Park, M., Jin, J. S., and Luo, S. (2006). Locating the optic disc in retinal images. In Proceedings of the International Conference on Computer Graphics, Imaging and Visualisation, pages 141-145. IEEE Computer Society. One citation in page 33.

Patton, N., Aslam, T. M., MacGillivray, T., Deary, I. J., Dhillon, B., Eikelboom, R. H., Yogesan, K., and Constable, I. J. (2006). Retinal image analysis: Concepts, applications and potential. Progress in Retinal and Eye Research, 25(1):99 - 127. One citation in page 43.

Perez-Rovira, A., MacGillivray, T., Trucco, E., Chin, K., Zutis, K., Lupascu, C., Tegolo, D., Giachetti, A., Wilson, P., Doney, A., and Dhillon, B. (2011). Vampire: Vessel assessment and measurement platform for images of the retina. In Engineering in Medicine and Biology Society,EMBC, 2011 Annual International Conference of the IEEE, pages 3391-3394. One citation in page 24 .

Philip, S., Fleming, A. D., Goatman, K. A., Fonseca, S., Mcnamee, P., Scotland, G. S., Prescott, G. J., Sharp, P. F., and Olson, J. A. (2007). The efficacy of automated "disease/no disease" grading for diabetic retinopathy in a systematic screening programme. British Journal of Ophthalmology, 91(11):1512-1517. 2 citations in pages 20 and 24.

Quinlan, J. R. (1993). C4.5: Programs for Machine Learning. Morgan Kaufmann Publishers Inc. One citation in page 135.

Qureshi, R. J., Kovacs, L., Harangi, B., Nagy, B., Peto, T., and Hajdu, A. (2012). Combining algorithms for automatic detection of optic disc and macula in fundus images. Computer Vision and Image Understanding, 116(1):138 - 145. One citation in page 33 . 
Ricci, E. and Perfetti, R. (2007). Retinal blood vessel segmentation using line operators and support vector classification. Medical Imaging, IEEE Transactions on, 26(10):1357-1365. 2 citations in pages 32 and 93.

Rodriguez, J., Kuncheva, L., and Alonso, C. (2006). Rotation forest: A new classifier ensemble method. Pattern Analysis and Machine Intelligence, IEEE Transactions on, 28(10):1619-1630. One citation in page 135.

Rogers, S. L., Tikellis, G., Cheung, N., Tapp, R., Shaw, J., Zimmet, P. Z., Mitchell, P., Wang, J. J., and Wong, T. Y. (2008). Retinal arteriolar caliber predicts incident retinopathy: the australian diabetes, obesity and lifestyle (ausdiab) study. Diabetes Care, 31(4):761-3. One citation in page 79 .

Rosenfeld, A. and Pfaltz, J. L. (1968). Distance functions on digital pictures. Pattern Recognition, 1(1):33-61. One citation in page 72.

Ruggeri, A., Grisan, E., and De Luca, M. (2007). An automatic system for the estimation of generalized arteriolar narrowing in retinal images. In Engineering in Medicine and Biology Society, 200\%. EMBS $200 \%$. 29th Annual International Conference of the IEEE, pages 6463-6466. 2 citations in pages 17 and 80 .

Russ, J. C. (2007). Image Processing Handbook. CRC Press, Inc., 5th edition. One citation in page 67.

Scheffer, T. (1999). Error Estimation and Model Selection. PhD thesis, Technischen Universität Berlin, School of Computer Science. One citation in page 127 .

Serra, J. (1982). Image Analysis and Mathematical Morphology, volume I. Ac. Press, London. One citation in page 34.

Singalavanija, A., Supokavej, J., Bamroongsuk, P., Sinthanayothin, C., Phoojaruenchanachai, S., and Kongbunkiat, V. (2006). Feasibility study on computer-aided screening for diabetic retinopathy. Japanese Journal of Ophthalmology, 50(4):361-366. One citation in page 19.

Sinthanayothin, C., Boyce, J. F., Cook, H. L., and Williamson, T. H. (1999). Automated localisation of the optic disc, fovea, and retinal blood vessels from digital colour fundus images. British Journal of Ophthalmology, 83:902-910. One citation in page 32 .

Sinthanayothin, C., Boyce, J. F., Williamson, T. H., Cook, H. L., Mensah, E., Lal, S., and Usher, D. (2002). Automated detection of diabetic 
retinopathy on digital fundus images. Diabetic Medicine, 19(2):105-112. One citation in page 19 .

Sofka, M. and Stewart, C. (2006). Retinal vessel centerline extraction using multiscale matched filters, confidence and edge measures. IEEE TMI, 25:1531-1546. One citation in page 32 .

Soille, P. (2003). Morphological Image Analysis: Principles and Applications. Springer-Verlag New York, Inc., 2nd edition. 6 citations in pages 34, 57, $58,82,83$, and 94 .

Staal, J., Abramoff, M., Niemeijer, M., Viergever, M., and van Ginneken, B. (2004). Ridge-based vessel segmentation in color images of the retina. Medical Imaging, IEEE Transactions on, 23(4):501 -509. 5 citations in pages $46,86,89,94$, and 98 .

Sun, K., Chen, Z., Jiang, S., and Wang, Y. (2011). Morphological multiscale enhancement, fuzzy filter and watershed for vascular tree extraction in angiogram. Journal of medical systems, 35(5):811-824. One citation in page 32 .

Telea, A. (2004). An image inpainting technique based on the fast marching method. Journal of Graphics Tools, 9(1):23-34. One citation in page 70.

Tramontan, L., Grisan, E., and Ruggeri, A. (2008). An improved system for the automatic estimation of the arteriolar-to-venular diameter ratio (avr) in retinal images. In Engineering in Medicine and Biology Society, 2008. EMBS 2008. 30th Annual International Conference of the IEEE, pages 3550-3553. 2 citations in pages 17 and 80.

Vasan, R., Beiser, A., Seshadri, S., and et al. (2002). Residual lifetime risk for developing hypertension in middle-aged women and men: The framingham heart study. JAMA, 287(8):1003-1010. One citation in page 16.

Verma, L., Prakash, G., and Tewari, H. K. (2002). Diabetic retinopathy: time for action. No complacency please! Bulletin of the World Health Organization, 80:419 - 419. One citation in page 3 .

Vincent, L. (1998). Minimal path algorithms for the robust detection of linear features in gray images. In Proceedings of the fourth international symposium on Mathematical morphology and its applications to image and signal processing, ISMM '98, pages 331-338. One citation in page 72. 
Walter, T. (2003). Application de la Morphologie Mathématique au diagnostic de la Rétinopathie Diabétique à partir d'images couleur. $\mathrm{PhD}$ thesis, École Nationale Supérieure des Mines de Paris. 2 citations in pages 54 and 82.

Walter, T. and Klein, J. C. (2002). A computational approach to diagnosis of diabetic retinopathy. In Proceedings of the 6th Conference on Systemics Cybernetics and Informatics (SCI), pages 521-526. 6 citations in pages $32,46,82,95,96$, and 97.

Walter, T., Klein, J. C., Massin, P., and Erginay, A. (2002). A contribution of image processing to the diagnosis of diabetic retinopathy-detection of exudates in color fundus images of the human retina. Medical Imaging, IEEE Transactions on, 21(10):1236-1243. 7 citations in pages $33,42,63$, 66, 106, 109, and 110 .

Wang, H., Hsu, W., Goh, K. G., and Lee, M. L. (2000). An effective approach to detect lesions in color retinal images. In Computer Vision and Pattern Recognition, 2000. Proceedings. IEEE Conference on, volume 2, pages 181-186 vol.2. One citation in page 18.

Welfer, D., Scharcanski, J., Kitamura, C. M., Pizzol, M. M. D., Ludwig, L. W., and Marinho, D. R. (2010). Segmentation of the optic disk in color eye fundus images using an adaptive morphological approach. Computers in Biology and Medicine, 40(2):124 - 137. 5 citations in pages ix, 33, 100, 107, and 109.

Witten, I. H., Frank, E., and Hall, M. A. (2011). Data Mining: Practical Machine Learning Tools and Techniques. The Morgan Kaufmann Series in Data Management Systems. Morgan Kaufmann, third edition edition. One citation in page 130.

Wong, T. and Mitchell, P. (2007). The eye in hypertension. The Lancet, 369(9559):425-435. One citation in page 16.

Wong, T. Y., Klein, R., Klein, B. E., Tielsch, J. M., Hubbard, L., and Nieto, F. (2001). Retinal microvascular abnormalities and their relationship with hypertension, cardiovascular disease, and mortality. Survey of Ophthalmology, 46(1):59 - 80. One citation in page 17 .

Wong, T. Y., Knudtson, M. D., Klein, R., Klein, B. E. K., Meuer, S. M., and Hubbard, L. D. (2004). Computer-assisted measurement of retinal vessel diameters in the beaver dam eye study: methodology, correlation between eyes, and effect of refractive errors. Ophthalmology, 111(6):1183-1190. 3 citations in pages 17,79 , and 81 . 
World Health Organization (2010a). Action plan for the prevention of blindness and visual impairment, 2009-2013. 2 citations in pages 15 and 119.

World Health Organization (2010b). Global status report on noncommunicable diseases. One citation in page 16.

World Health Organization (2013). Universal eye health: a global action plan 2014-2019. One citation in page 3 .

Wu, C., Kang Derwent, J., and Stanchev, P. (2009). Retinal vessel radius estimation and a vessel center line segmentation method based on ridge descriptors. Journal of Signal Processing Systems, 55(1-3):91-102. One citation in page 32 .

Xu, J., Chutatape, O., Sung, E., Zheng, C., and Chew Tec Kuan, P. (2007). Optic disk feature extraction via modified deformable model technique for glaucoma analysis. Pattern Recognition, 40:2063-2076. One citation in page 33 .

Yang, Z. and Ai, H. (2007). Demographic classification with local binary patterns. In Lee, S.-W. and Li, S., editors, Advances in Biometrics, volume 4642 of Lecture Notes in Computer Science, pages 464-473. Springer Berlin Heidelberg. One citation in page 120.

Yun, W. L., Acharya, U. R., Venkatesh, Y., Chee, C., Min, L. C., and Ng, E. (2008). Identification of different stages of diabetic retinopathy using retinal optical images. Information Sciences, 178(1):106 - 121. One citation in page 18 .

Zabihi, S., Delgir, M., and Pourreza, H.-R. (2010). Retinal vessel segmentation using color image morphology and local binary patterns. In Machine Vision and Image Processing (MVIP), 6th Iranian, pages 1-5. One citation in page 120 .

Zana, F. and Klein, J. C. (2001). Segmentation of vessel-like patterns using mathematical morphology and curvature evaluation. IEEE Transactions on Image Processing, 10:1010-1019. 4 citations in pages 32, 49, 90, and 92.

Zhang, X., Thibault, G., Decencière, E., Quellec, G., Cazuguel, G., Erginay, A., Massin, P., and Chabouis, A. (2012). Spatial normalization of eye fundus images. In ISBI 2012 : 9th IEEE International Symposium on Biomedical Imaging, Barcelone, Spain. IEEE. One citation in page 125. 
Zheng, Y., Hijazi, M. H. A., and Coenen, F. (2012). Automated disease / no disease: Grading of age-related macular degeneration by an image mining approach. Investigative Ophthalmology \& Visual Science, 53(13):8310-8. One citation in page 130 . 